$10-2015$

\title{
Archeological Investigation at Yanaguana Garden in Hemisfair Park, San Antonio, Bexar County, Texas
}

Ross C. Fields

Prewitt and Associates, Inc.

Aaron R. Norment

AmaTerra Environmental, Inc.

Amy E. Dase

Prewitt and Associates, Inc.

Follow this and additional works at: https://scholarworks.sfasu.edu/ita

Part of the American Material Culture Commons, Archaeological Anthropology Commons, Environmental Studies Commons, Other American Studies Commons, Other Arts and Humanities Commons, Other History of Art, Architecture, and Archaeology Commons, and the United States History Commons

Tell us how this article helped you.

This Article is brought to you for free and open access by the Center for Regional Heritage Research at SFA ScholarWorks. It has been accepted for inclusion in Index of Texas Archaeology: Open Access Gray Literature from the Lone Star State by an authorized editor of SFA ScholarWorks. For more information, please contact cdsscholarworks@sfasu.edu. 
Archeological Investigation at Yanaguana Garden in Hemisfair Park, San Antonio, Bexar County, Texas

\section{Creative Commons License}

\section{(c) (1) $\Theta(9$}

This work is licensed under a Creative Commons Attribution-NonCommercial-No Derivative Works 4.0 International License. 


\title{
ARCHEOLOGICAL INVESTIGATIONS AT YANAGUANA GARDEN IN HEMISFAIR PARK, SAN ANTONIO, BEXAR COUNTY, TEXAS
}

\author{
by \\ Ross C. Fields \\ Aaron R. Norment \\ and \\ Amy E. Dase \\ Principal Investigator: Ross C. Fields \\ REPORTS OF INVESTIGATIONS, NUMBER 179 \\ submitted to \\ Adams Environmental, Inc. \\ San Antonio, Texas \\ and \\ City of San Antonio \\ Transportation and Capital Improvements
}

by

Prewitt and Associates, Inc.

Cultural Resources Services

Austin, Texas

PAI Nos. 214006, 214016, 214022, 214028, 214029, and 214033

October 2015

TEXAS ANTIQUITIES PERMIT NO. 6846 



\section{TABLE OF CONTENTS}

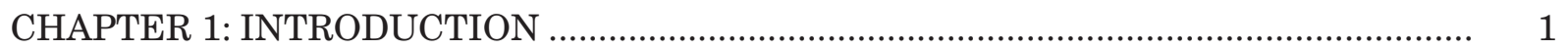

Summary of Work Orders...................................................................................

The HemisFair Park Master Plan................................................................................. 3

The Yanaguana Garden Development Plan ........................................................... 5

CHAPTER 2: BACKGROUND INFORMATION ON YANAGUANA GARDEN ................... 9

Historical Background....................................................................................... 9

Known Sites and Previous Archeological Investigations ........................................ 11

Disturbance Factors...........................................................................................

Overall Recommendations .............................................................................. 16

CHAPTER 3: WORK ACCOMPLISHED .................................................................... 21

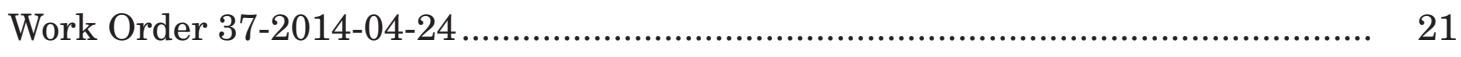

Work Order 39-2014-07-24 ………………………………………………….... 23

Work Order 43-2014-08-04 ……………………………………………………. 24

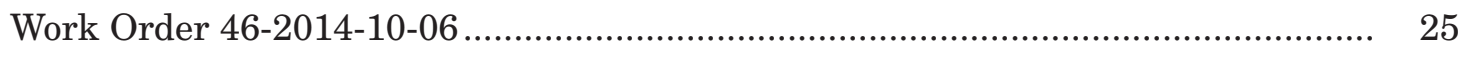

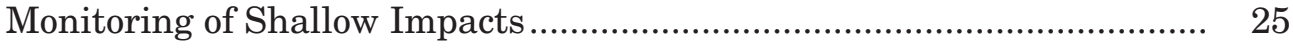

Monitoring of Deep Impacts..................................................................... 31

CHAPTER 4: RESULTS OF ARCHEOLOGICAL INVESTIGATIONS .............................. 39

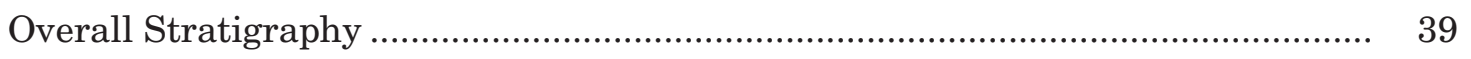

Site 41BX8, Acequia Madre............................................................................... 42

Site 41BX2068, Trash Pit ............................................................................ 46

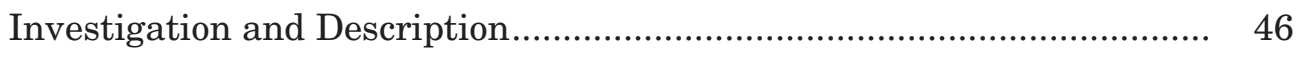

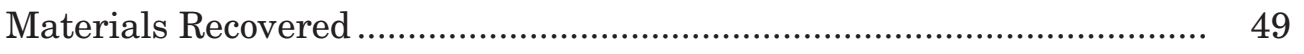

Chronology and Associations ................................................................ 59

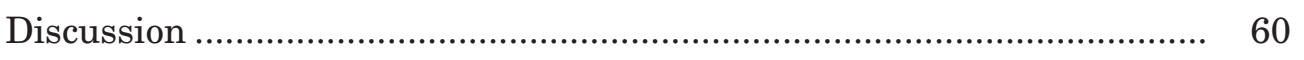

Archival Research................................................................................ 61

Assessment....................................................................................... 66

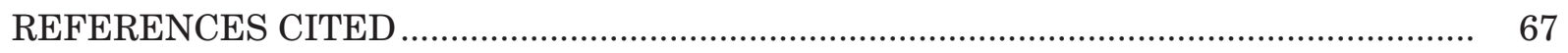


APPENDIX B: Data Compilation for Archeological Planning at HemisFair Park, San Antonio, Texas (digital format only)

APPENDIX C: Recommendations Concerning Treatment of Archeological and Historical Resources, HemisFair Internal Streets Improvements, San Antonio, Bexar County, Texas (digital format only) 


\section{LIST OF FIGURES}

1. Map from the 2012 HemisFair Park Master Plan showing proposed development plan

2. Development plan for Yanaguana Garden ........................................................... 6

3. Plan of the Yanaguana Garden area showing nearby historic buildings .................. 10

4. 1912 Sanborn Fire Insurance Company map of the Yanaguana Garden area .......... 12

5. Plan showing known archeological sites and investigations prior to $2014 \ldots \ldots \ldots \ldots \ldots . . . . .13$

6. Aerial view to the southwest of the project area and adjoining areas showing the level of disturbance associated with construction of HemisFair '68 .....

7. Ground-level view of disturbance associated with HemisFair ' 68 construction

8. Plan showing locations of now-demolished HemisFair '68 temporary buildings in and adjacent to the Yanaguana Garden project area.

9. Plan of the project area showing areas investigated archeologically.....

10. Examples of construction activities that caused shallow subsurface impacts 32

11. Examples of construction activities that caused shallow subsurface impacts

12. View to the southwest of shallow impacts caused by grading for walkways at the north end of the project area.

13. View to the northeast of Bobcats excavating 4-ft-deep new restroom pad

14. Photograph of excavator removing the upper part of one of six deep (10-12 ft) concrete piers

15. Examples of construction activities that caused deep subsurface impacts

16. View of deep excavation required for placement of casing to hold sewer line that was bored beneath the acequia alignment in the south part of the project area.....

17. View to the north of the trench dug to remove contaminated soils beneath the parking lot between the Eager and Pereida houses showing subsurface stratigraphy

18. View to the southeast of intersecting sewer line bore pit and Bioswale Pit 3 showing subsurface stratigraphy

19. View to the southeast of the west face of the acequia wall segment in Trench PAI-1

20. View to the northeast of the north wall of Trench PAI-3

21. View to the southeast of the west face of the acequia wall segment in Trench PAI-4

22. Profile and photograph of the south wall of the storm sewer line trench in which trash pit feature at 41BX2068 was found

23. View of the east wall of the 1x1-m unit excavated into the 41BX2068 trash pit. 
24. Examples of the two styles of ginger beer bottles recovered from the 41BX2068 trash pit 50

25. Whiteware fragments with makers' marks from the 41BX2068 trash pit 52

26. Additional decorated ceramic styles and glass tumbler from the 41BX2068 trash pit 54

27. Clothing-related artifacts recovered from the 41BX2068 trash pit 55

28. Activities-related artifacts recovered from the 41BX2068 trash pit 57

29. Map showing Lot 5, Block 5 of City Block 890 bounded by Water Street and the Alamo Ditch. 62

30. Horace Hammant and Pauline Volpert Hugman ................................................ 64

A.1. Example of bullet hole in a bovid scapula 


\section{LIST OF TABLES}

1. Summary of work order products..................................................................... 2

2. Daily log of archeological construction monitoring activities under Work Order 46-2014-10-66 ............................................................................ 26

3. Summary of deeper sediment profiles at Yanaguana Garden ................................. 40

4. Summary of historic sites investigated during the Alamodome and Henry B. Gonzalez Convention Center projects that were used for comparison

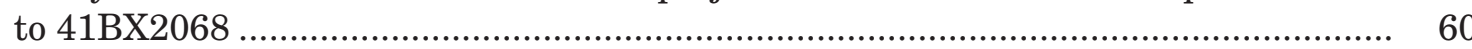

A.1. List of vertebrate faunal taxa from 41BX2068 ..................................................... 79

A.2. Comparison of faunal assemblage from 41BX2068 with those from roughly contemporaneous sites 41BX897 and 41BX883 



\section{CHAPTER 1: INTRODUCTION}

This report describes archeological efforts done under six work orders for the development of Yanaguana Garden at HemisFair Park in downtown San Antonio, Texas. All of the projects were done by Prewitt and Associates, Inc. (PAI), for Adams Environmental, Inc. (AEI), and the City of San Antonio, Transportation and Capital Improvements (CoSA-TCI), under Texas Antiquities Permit No. 6846 (issued April 14, 2014). As described below, the Yanaguana Garden project is the first phase of a planned redevelopment of HemisFair Park for mixed-use purposes. Planning for how to deal with cultural resources during this redevelopment began in 2012 when PAI prepared two reports summarizing known archeological and historical resources and providing recommendations for future work (Dase 2013; Fields and McWilliams 2012). Almost all of the subsequent work reported here dealt strictly with the permitted archeological investigations, with limited effort going toward historical resources under a single work order.

\section{SUMMARY OF WORK ORDERS}

The first work order (WO 34-2014-02-26; PAI 214006) commenced on February 27, 2014, and consisted of two main tasks. One entailed obtaining an antiquities permit for all archeological work to be done in connection with the development of Yanaguana Garden, and the other involved updating and completing the archeological file search, literature review, and data compilation done in 2012. The latter task included all of HemisFair Park but provided the most detail on the southwest corner where Yanaguana Garden would be; the result of that effort is included as Appendix B to this report.

The second work order (WO 37-2014-04-24; PAI 214016) was issued on April 28, 2014, and dealt solely with archeological trenching, and subsequent monitoring, for construction of an emergency service drive and storm drain to be built at the north edge of Yanaguana Garden in advance of park construction. The third work order (WO 39-2014-07-24; PAI 214022), issued on July 25, 2014, also was for an activity that preceded park construction; it consisted of archeological monitoring of the removal of lead-contaminated sediments at two locations in the west-central and northeast parts of the park.

The fourth work order (WO 43-2014-08-04; PAI 214028) was issued on August 4, 2014, and had two tasks. One involved successful archeological trenching to search for the remains of a known site within the Yanaguana Garden project area, the Acequia Madre (or Alamo Acequia; 41BX8). The other consisted of 
providing a comprehensive assessment of the known and potential archeological and historical resources that could be impacted by the Yanaguana Garden project and recommendations for appropriate investigations to deal with those resources.

The fifth work order (WO 44-2014-08-18; PAI 214029) was issued on August 18, 2014. Most of it involved providing an assessment of known and potential archeological and historical resources that could be impacted by proposed street and utilities improvements (the Internal Streets Project) along the north and east edges of the project area; because these improvements will be outside Yanaguana Garden proper and any archeological work done in connection with them will be under a separate permit, they are not addressed in the body of this report; the result of that study is included as Appendix $\mathrm{C}$ to this report, though. This work order also included efforts to finalize the Yanaguana Garden-specific data compilation done under the first work order (see Appendix B).

The sixth work order (WO 46-2014-10-06; PAI 214033) was issued on October 6,2014 . It involved archeological monitoring of construction activities at Yanaguana Garden from their inception on October 13 through December 2014, when another contractor assumed responsibility for monitoring under a different antiquities permit. It also involved analysis and reporting of data generated by all six work orders to create this report.

The first five work orders resulted in a compilation of archeological and other data to aid in planning work at Yanaguana Garden, three preliminary reports on archeological fieldwork, and one document reporting overall recommendations for the project area, in addition to the antiquities permit application (Table 1). All of these documents are synthesized in this report, along with the methods and results of monitoring done under the sixth work order (for which a preliminary report was not produced).

Table 1. Summary of work order products

\begin{tabular}{|c|c|c|}
\hline Work Order & Product & Date \\
\hline WO 34-2014-02-26 & $\begin{array}{l}\text { antiquities permit application } \\
\text { Data Compilation for Archeological Planning at HemisFair Park, San } \\
\text { Antonio, Texas (Appendix B to this report) }\end{array}$ & $\begin{array}{l}\text { March } 2014 \\
\text { June } 2014\end{array}$ \\
\hline WO 37-2014-04-24 & $\begin{array}{l}\text { Preliminary Report on Archeological Investigations for the HemisFair } \\
\text { Park Yanaguana Garden North Emergency Service Drive, San Antonio, } \\
\text { Bexar County, Texas }\end{array}$ & July 2014 \\
\hline WO 39-2014-07-24 & $\begin{array}{l}\text { Preliminary Report on Archeological Monitoring of Removal of } \\
\text { Contaminated Soils at Proposed Yanaguana Garden at HemisFair Park, } \\
\text { San Antonio, Bexar County, Texas }\end{array}$ & October 2014 \\
\hline WO 43-2014-08-04 & $\begin{array}{l}\text { Preliminary Report on Archeological Trenching to Search for the Acequia } \\
\text { Madre at Proposed Yanaguana Garden at Hemisfair Park, San Antonio, } \\
\text { Bexar County, Texas } \\
\text { Recommendations Concerning Treatment of Archeological and Historical } \\
\text { Resources at Proposed Yanaguana Garden, HemisFair Park, San Antonio, } \\
\text { Bexar County, Texas }\end{array}$ & $\begin{array}{l}\text { August } 2014 \\
\\
\text { September } \\
2014\end{array}$ \\
\hline WO 44-2014-08-18 & Appendixes B and C to this report & - \\
\hline WO 46-2014-10-06 & this report & May 2015 \\
\hline
\end{tabular}


As noted, only one work order involved historical resources, and that consisted of incorporating such resources into the Yanaguana Garden recommendations document. That document noted that the following historical resources are contributing components in a National Register-eligible historic district in the immediate vicinity of the Yanaguana Garden project area: Beethoven Hall; the O.K. Bar; the Eager house and its associated carriage house and dependency; the AcostaHalff, Kampmann-Halff, Amaya, Smith, Solis, Pereida, Koehler, and Espinoza houses; and the Celanese Fountain. Only the Celanese Fountain would be impacted directly by the project, however, and additional research indicated that its National Register status should be changed to a noncontributing resource to the district. Review of 1966 site plans, a 1974 map, aerial images from 1966, 1973, 1986, and 2004, and photographs from 1968 and 2012 showed that the fountain was moved from its original position closer to South Alamo Street near the north side of the presentday location of the O.K. Bar. By 1970, the fountain had been vandalized, according to an August 27 article in the San Antonio Express. The base was broken, and the original two-tier center piece had been demolished. In 2012, a modern three-tiered center piece had been installed. The fountain was moved sometime between 1986 and 2004. As a result, it no longer retained integrity of location, design, materials, workmanship, or feeling.

\section{THE HEMISFAIR PARK MASTER PLAN}

The 2012 file search, literature review, and data compilation covered a 104acre area specified for development in the HemisFair Park Master Plan (HemisFair Park Area Redevelopment Corporation 2012). That included the full 96-acre extent of HemisFair Park (bounded by South Alamo Street on the west, Market Street on the north, South Bowie Street on the east, and East Cesar Chavez Boulevard on the south (and thus encompassing the Henry B. Gonzalez Convention Center and adjoining buildings), plus 8 acres south of East Cesar Chavez Boulevard (Figure 1).

As presented in the Master Plan, the primary components of the project would be the following: (1) reestablishing a portion of the pre-1968 (i.e., pre-HemisFair '68) street grid; (2) creation of approximately 33 acres of parks and open spaces; (3) reconfiguring some of the current civic space; (4) new development for mixed civic, commercial, and residential uses on about 35 acres; and (5) construction of parking structures. Reestablishing the pre-1968 street grid would involve reconstructing on the their original locations Goliad Street (or East Nueva Street, as of the end of 2014) from South Alamo to South Bowie Streets and the following perpendicular streets from East Cesar Chavez Boulevard one-and-a-half to two-and-a-half blocks northward to the Henry B. Gonzalez Convention Center: Water Street, Matagorda Street, and Indianola Street. Several other streets to be added are not on the pre1968 grid, or are offset from it slightly: the northeast and northwest segments (total length $=270 \mathrm{ft}$ ) of a drop-off loop at the northwest corner of the Goliad Street-North Water Street intersection (no previous street here); a 670-ft-long east-southeasttrending dogleg off the north end of Indianola Street (labeled North Street in the Master Plan; no previous street here); a 940-ft-long street (labeled South Street in the Master Plan) running southeastward from Indianola Street to the east edge 


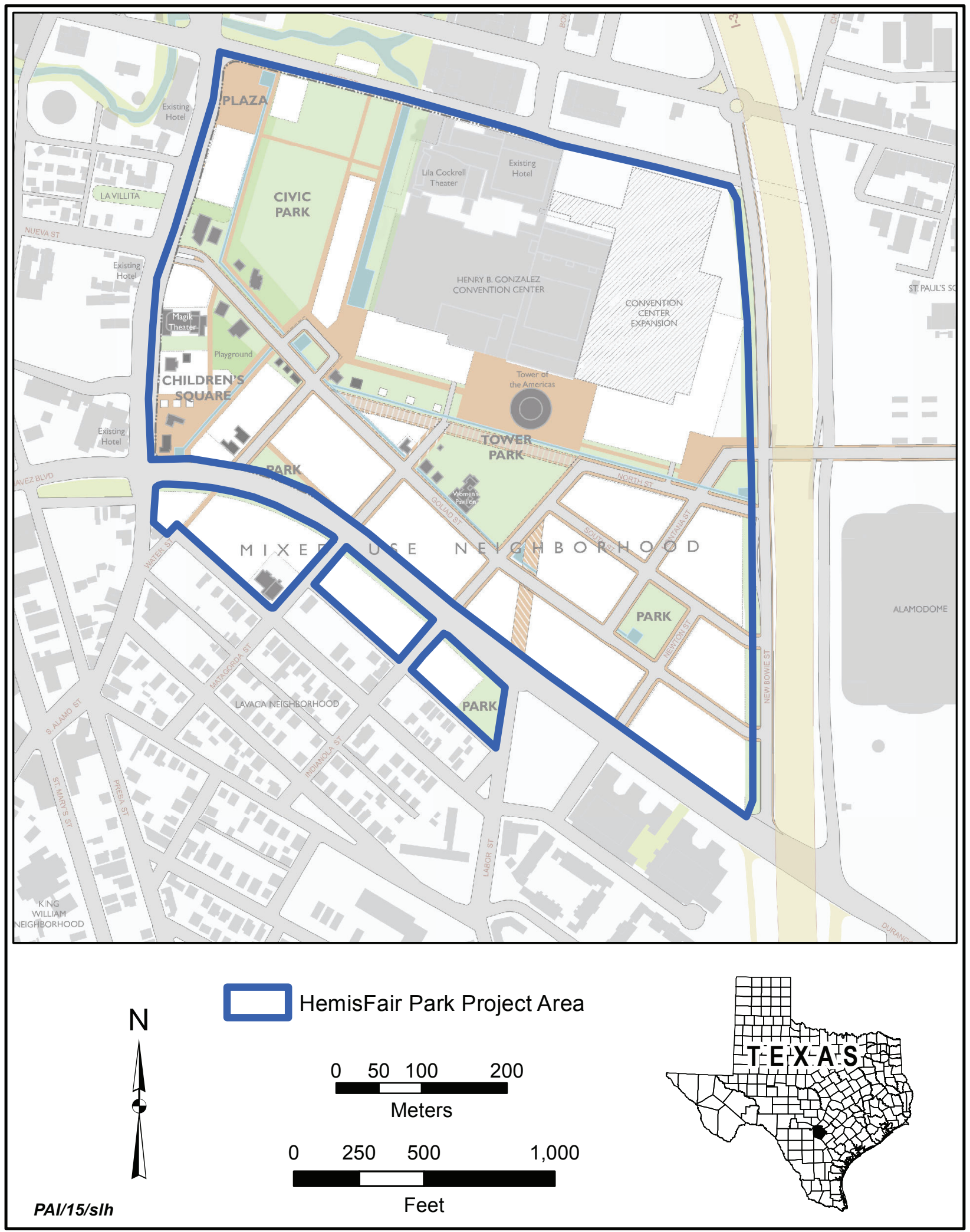

Figure 1. Map from the 2012 HemisFair Park Master Plan showing proposed development plan. 
of the project area (partly aligns with pre-1968 South Street but is slightly more east-west); a 910-ft-long street (labeled Montana Street in the Master Plan) running two-and-a-half blocks northeastward from Goliad Street east of Indianola Street (partly aligns with pre-1968 Newton Street but is slightly more north-south); and a 520-ft-long street (labeled Newton Street in the Master Plan) running two blocks northeastward from East Cesar Chavez Boulevard east of new Montana Street (partly aligns with Santa Clara Street but is slightly more north-south).

Among the proposed parks and open spaces were the following: (1) an 11.6acre civic park and plaza where the western part of the convention center is today; (2) a 6.7-acre park and plaza at the Tower of Americas and south to Goliad Street; (3) a 1.8-acre promenade running west from the tower park and plaza; (4) a 1-acre gateway transit park and plaza at the east-central edge of the project area; (5) a 0.8 -acre children's square and playground in the southwest corner of the project area (now known as Yanaguana Garden); and (6) three 0.4-1.0-acre neighborhood parks, two north of East Cesar Chavez Boulevard and one south of it. Reconfiguring current civic space would focus on the Henry B. Gonzalez Convention Center. The western part would be demolished for creation of a civic park and plaza, and the existing center would be expanded to the east almost all the way to Interstate Highway 37 (this was well under way as of the end of December 2014).

The new development for mixed civic, commercial, and residential uses would occur on about 35 acres, 30 acres north of East Cesar Chavez Boulevard (mostly south of the convention center and Tower of the Americas) and 5 acres south of it. To support the various improvements, five new parking structures were proposed. Two would be large underground structures, one at the corner of South Alamo and Market Streets and the other between Goliad Street and East Cesar Chavez Boulevard from Matagorda Street west beneath Water Street. The other three would be above-ground structures incorporated into new mixed-use developments adjoining the existing and new convention center.

\section{THE YANAGUANA GARDEN DEVELOPMENT PLAN}

After the master plan was completed, a detailed plan was developed for Yanaguana Garden (previously referred to as the Children's Square and the PlayEscape) in the southwest corner of the HemisFair project area, bounded on the west by South Alamo Street, on the south by East Cesar Chavez Boulevard, on the north by HemisFair Plaza Way (old Goliad Street and referred to as East Nueva Street as of the end of 2014), and on the east by an existing parking lot and historic houses adjacent to the original route of Water Street (Figure 2). This plan directed all of the archeological investigations reported here.

Central to the proposed development will be a paved, pergola-covered walkway that will run southwest-northeast between the East Cesar Chavez Boulevard gateway into the park and Hemisfair Plaza Way (future East Nueva Street). Paved plazas will be at both ends of the promenade, and at its center will be a paved play square. These features will divide the project area into quadrants of unequal size. Much of the southeast quadrant will be devoted to water and sand play 


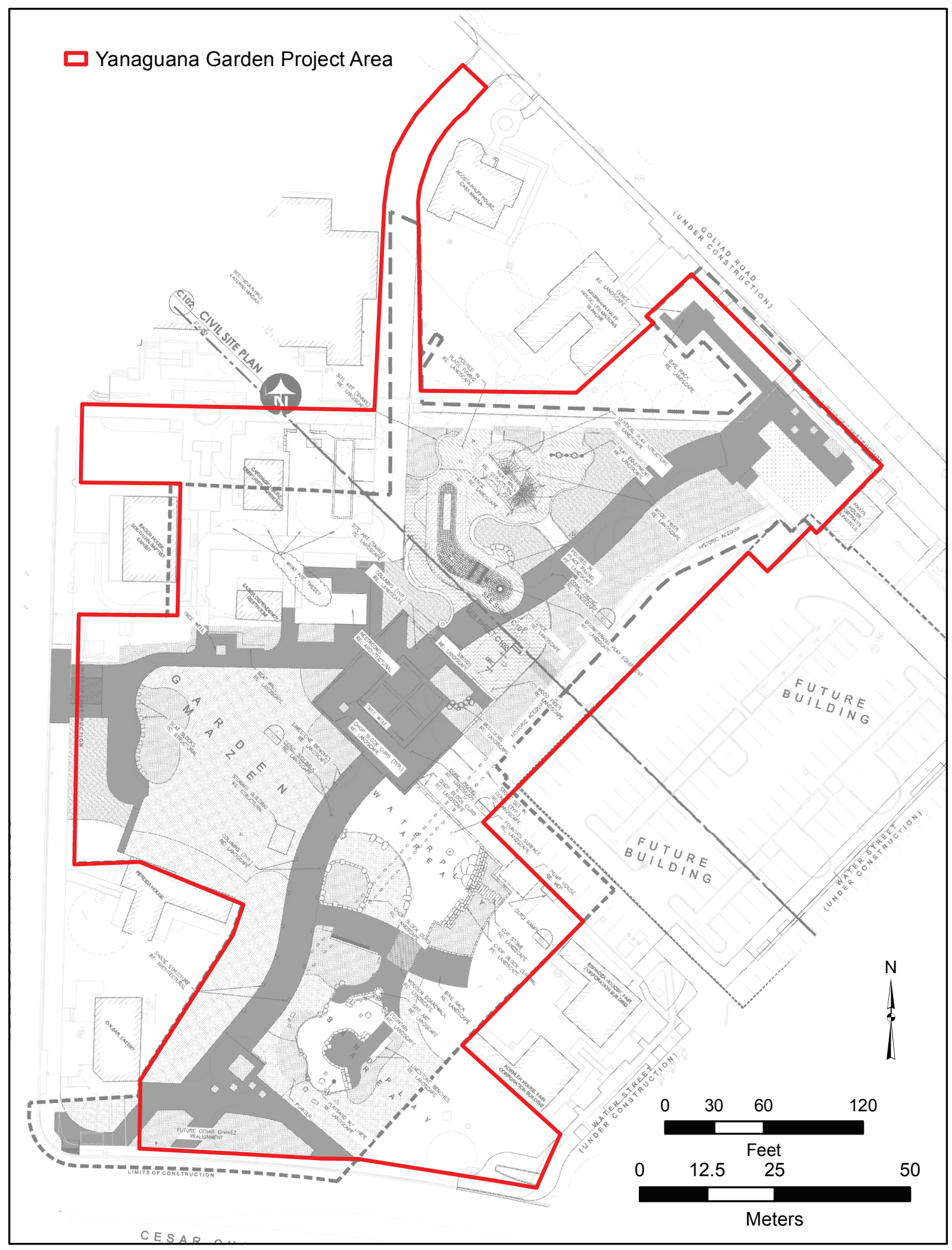

Figure 2. Development plan for Yanaguana Garden. 
areas and adjacent lawns and planted areas; a small pump house will be associated with the water play area. Most of the narrow northeast quadrant will be planted areas or lawn flanking the reconstructed segment of the Acequia Madre, with an area of playground equipment at its southwest corner. The northwest quadrant will contain a new restroom facility and a remodeled restroom, an area for playground equipment, play sculpture, a games porch with concrete play tables (chess, foosball, and table tennis), and planted areas. The southwest quadrant will be mostly a multipurpose play lawn adjacent to the South Alamo Street plaza gateway into the park with some planted areas (called Garden Maze in Figure 2). The total horizontal Area of Potential Effects (APE) will be 3.3 acres. 



\section{CHAPTER 2: BACKGROUND INFORMATION ON YANAGUANA GARDEN}

\section{HISTORICAL BACKGROUND}

The area of Yanaguana Garden, i.e., the southwest corner of HemisFair Park, is sometimes referred to as the historic triangle because so many late-nineteenthcentury and early-twentieth-century buildings were preserved there, most in place but a few moved in or reconstructed, when HemisFair '68 was built. These include the following: the Acosta-Halff and Kampmann-Halff houses on the south side of Goliad Street (now East Nueva Street) at the north edge of Yanaguana Garden and the Amaya, Smith, Solis, and Kusch houses not far to the southeast along that street; Schultze Store and the Sweeney-Tynan, Schultze, and Halff houses in this same area on the north side of Goliad Street; the Espinoza and Koehler houses on the west side of old Water Street at the southeast corner of Yanaguana Garden; and O.K. Bar, the Pereida house, the Eager house and its associated dependency and carriage house, and Beethoven Hall on South Alamo Street at the west edge of Yanaguana Garden (Figure 3). These buildings reflect increased development of this section of downtown San Antonio after the Civil War, with mostly residential buildings and some commercial ones on the triangle of land bounded by South Alamo, Goliad, and Water Streets; the buildings faced these streets, and their lots backed up to the ditch known as the Acequia Madre.

The Acequia Madre is an important part of the early history of the area. It supplied water for irrigating agricultural fields and for consumption by people and livestock to the lower fields (labor de abajo) of Mission San Antonio de Valero, starting in the 1720s and continuing until 1793, when the mission was secularized (Fox and Cox 1990:1). The main north-south road connecting Mission Valero the Alamo) just north of the project area with Mission Concepcion to the south during the Spanish colonial period followed today's South Alamo Street, and La Bahia Road (the road to Presidio La Bahia near Goliad) ran southeast from South Alamo Street following what later would become Goliad Street. Both roads are evident on early maps of the area.

The area probably continued to be used for agricultural purposes after it was subdivided for settlement in 1793, and it appears that it was occupied intensively only after it was subdivided again in the latter decades of the 1800s. Historic maps attesting to this increase in development include the 1873 and 1886 Augustus Koch bird's-eye views of San Antonio (Koch 1873, 1886) and Sanborn maps dating to 1892, 1896, and 1912 (Sanborn Fire Insurance Company 1892, 1896, 1912). By 1912 , all of the lots in the historic triangle had buildings on them, mostly dwellings 


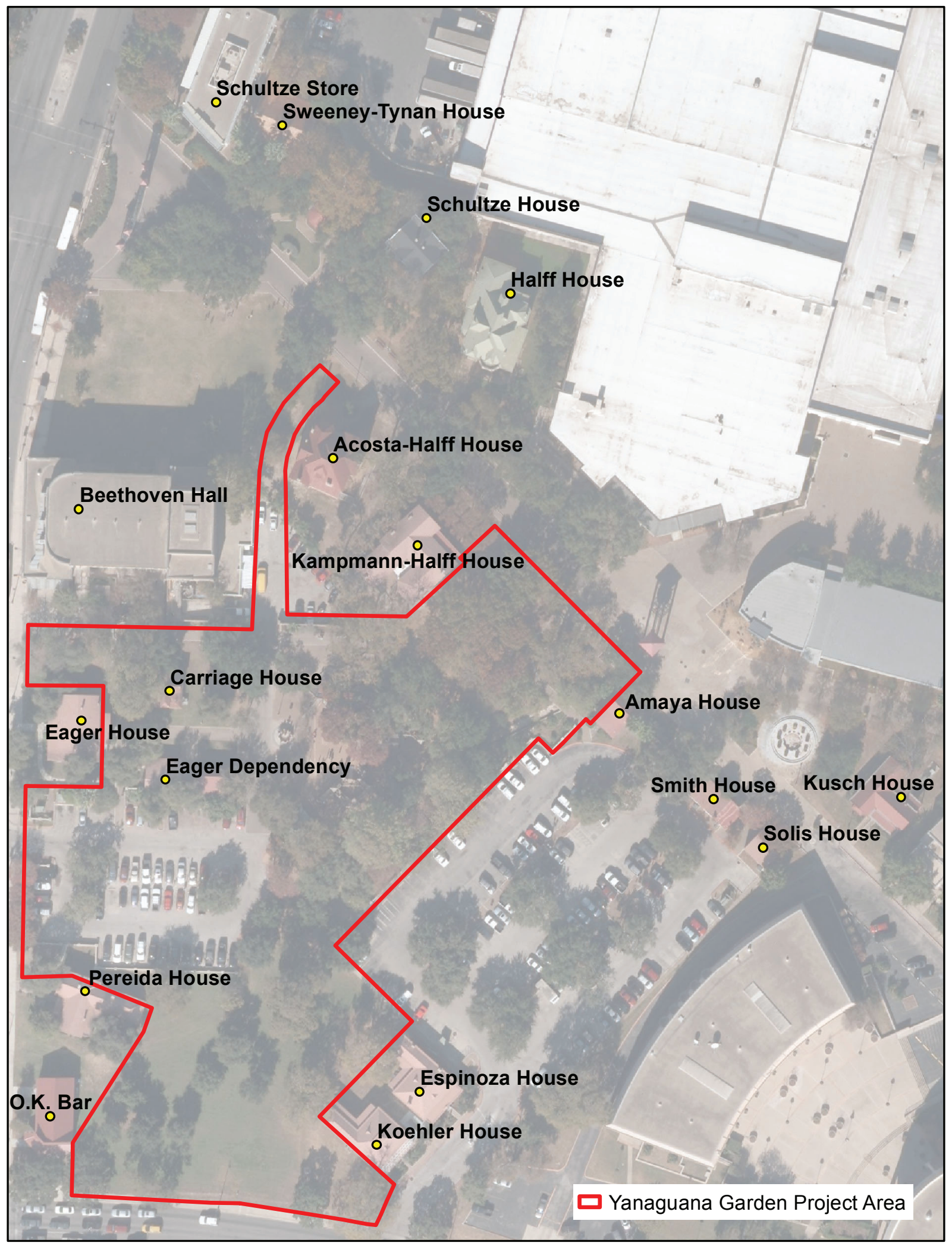

Figure 3. Plan of the Yanaguana Garden area showing nearby historic buildings. 
and nearby outbuildings but also a few commercial establishments and Beethoven Hall (German singing-society concert hall) and associated bowling alley (Figure 4).

The Acequia Madre ditch also is evident on these late-nineteenth- and earlytwentieth-century maps, indicating that it endured even after its agricultural function ceased. It continued to supply water and helped with drainage, but maintenance difficulties, sanitation problems posed by standing water, and construction of new city water works (1878) and sewer (1890s) systems eventually led to abandonment of the ditch (Cox 2005:60,67). In 1905, the city concluded that the Acequia Madre should be filled (Cox 2005:69-70), although both map and archeological evidence indicate that the section of it in the Yanaguana Garden project area remained open for some time after this.

Over the first half of the twentieth century, the character of this part of downtown San Antonio changed. Fewer houses were occupied by owners, and properties became run down, making the area ripe for redevelopment and urban renewal (Fox and Cox 1990:1). This led to choice of the site for HemisFair '68, construction of which started in 1966.

\section{KNOWN SITES AND PREVIOUS ARCHEOLOGICAL INVESTIGATIONS}

Prior to the work reported here, there were three recorded archeological sites within the Yanaguana Garden project area: 41BX8 (Acequia Madre), 41BX982 (Heubaum house foundation), and 41BX588 (relocated historic carriage house behind the Eager House). Seven other sites are immediately adjacent (Figure 5). Six of these are historic houses: 41BX585 (Acosta-Halff house), 41BX586 (KampmannHalff house), 41BX587 (Eager house), 41BX591 (Pereida house), 41BX592 (Koehler house), and 41BX593 (Espinoza house). And one, 41BX584, is historic Beethoven Hall. Only 41BX8 and 41BX982 have been investigated archeologically. The other eight sites were given trinomial designations based solely on the presence of extant historic buildings.

Site 41BX8 was investigated first in 1966, when Schuetz (1970) exposed and removed the fill from a 95-ft-long, partially preserved, rock-lined segment of the Acequia Madre so that it could be integrated into one of the HemisFair '68 pavilions; this was along the northeast edge of the project area (see Figure 5). This excavation determined that the rock walls of the acequia, the tops of which were visible at the surface in places, extended $5.2 \mathrm{ft}$ below the ground surface and that the ditch was $6.3 \mathrm{ft}$ wide. The limestone block walls were 10-14 inches thick, originally consisted of five courses of stone set in dry sandy mortar, and sat on $0.6 \mathrm{ft}$ of compacted mud, which rested on caliche; the mud and caliche were interpreted as in situ natural deposits (Schuetz 1970:4,5). The rock walls reportedly were not part of the original ditch construction, but were added in the mid-nineteenth century (Fox and Cox 1990:1). The bottom of the acequia was not lined with rocks. The ditch generally had three zones of fill. Below "several feet of recent fill," Zone 1 ( $0.6 \mathrm{ft}$ thick) consisted of "loose, black soil mixed with artifacts," and Zone 2 ( $1 \mathrm{ft}$ thick) was "characterized by gravels, often containing corroded iron objects, and a concentration of turn-of- 


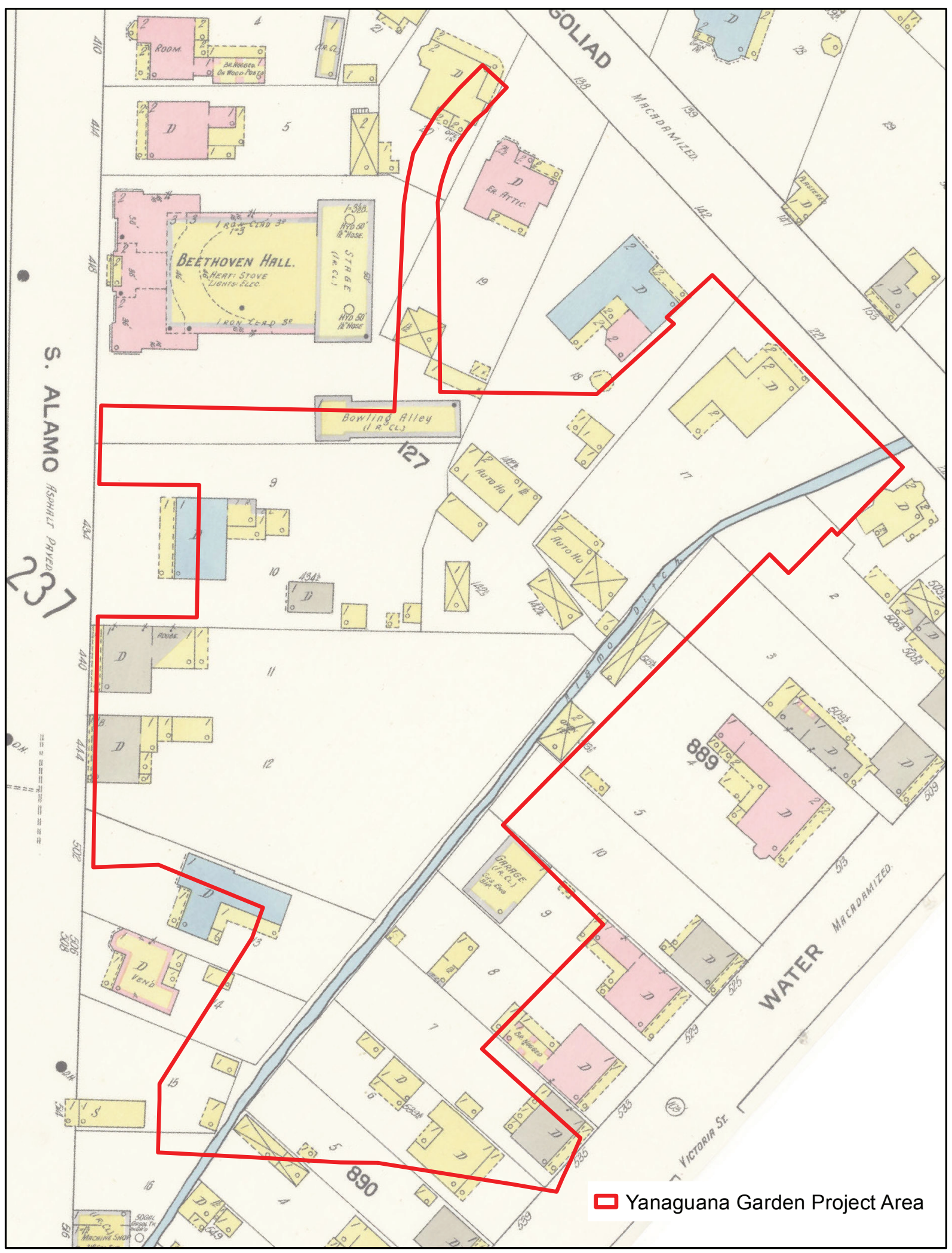

Figure 4. 1912 Sanborn Fire Insurance Company map of the Yanaguana Garden area. 


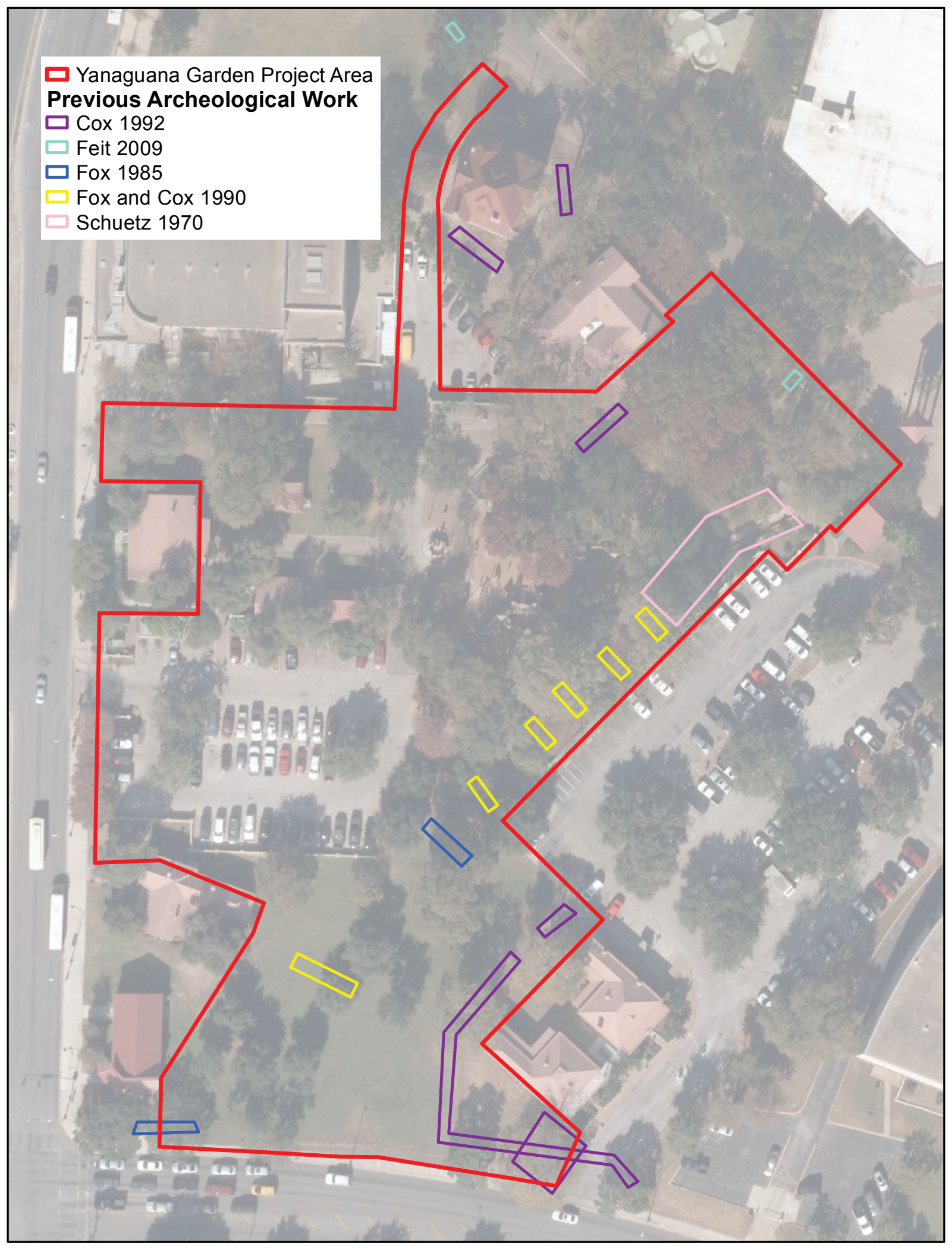

Figure 5. Plan showing known archeological sites and investigations prior to 2014. Site locations are not shown in report copies for public distribution. 
the-century artifacts" (Schuetz 1970:3-4). The excavations yielded a large collection of ceramic, glass, metal, and other artifacts, almost all of which appeared to date between the 1890s and 1930s. The only exceptions were 16 Spanish colonial artifacts, which were found in both Zones 1 and 2 (Schuetz 1970:7-13). Schuetz (1970:7) concluded that the "irrigation system was kept scrupulously clean during the Spanish colonial period, and was filled only after the Acequia was closed in the early 1890s."

In 1983-1984, Fox (1985) dug two trenches in a search for the Acequia Madre south of the segment documented by Schuetz (see Figure 5). One trench was in the proposed water play area at Yanaguana Garden. It identified the east rock wall of the acequia at $2.7 \mathrm{ft}$ below the surface beneath introduced fill and, about $6 \mathrm{ft}$ to the west, limestone rubble perhaps from the largely destroyed west wall at $3.2 \mathrm{ft}$. The ditch contained introduced caliche fill above dark brown clay with artifacts dating to the late-nineteenth and early-twentieth centuries to a depth of at least $5 \mathrm{ft}$ (Fox 1985:7, 10). The other trench was at the south edge of the Yanaguana Garden project area near where the East Cesar Chavez Boulevard gateway will be; the acequia was not positively identified there.

In 1989, Fox and Cox (1990) expanded on Fox's earlier work by excavating six trenches to look for the portion of the acequia south of the segment documented by Schuetz (see Figure 5). All six trenches exposed the filled acequia beneath 1.0-2.5 ft of introduced fill, with five of them containing the remains of the east wall of the ditch at 1.1-2.6 ft and one having limestone rubble perhaps marking the location of the west wall $6.5 \mathrm{ft}$ from east wall (Fox and Cox 1990:4-10). One trench also exposed two cedar posts probably representing an outbuilding outside the ditch alignment. Artifacts recovered from the ditch fill all represented early-twentiethcentury household goods (Fox and Cox 1990:10). The five trenches closest to Schuetz's excavations were used, along with the results of the 1966 work, to reconstruct the segment of the acequia that is there today. The other trench was southwest of there.

Site 41BX982, at the southeast corner of the project area, was identified in 1992 during monitoring by I. Waynne Cox of the excavation of approximately $270 \mathrm{ft}$ of utility trenches that ran along the south side of the Koehler (41BX592) house and just west of the Koehler and Espinoza (41BX593) houses (see Figure 5). A limestone foundation about $2 \mathrm{ft}$ high was found at a depth of $1 \mathrm{ft}$, below introduced fill, next to the southeast corner of the Koehler house, and apparently enough of this foundation was exposed to determine that it related to a house that measured $36 \times 28 \mathrm{ft}$, with porches adding another $6 \mathrm{ft}$ to the front and back. Archival research identified this as the 1859-1864 Heubaum house, which reportedly was demolished in 1964. Details of the excavations and findings are scant, though, because they were never fully written up, with sources on the work limited to the site form at the Texas Archeological Research Laboratory and a description and map reported by Johnson and Cox (1995:10-11).

Other than the investigations at 41BX8 and 41BX982, only two archeological studies had been done at Yanaguana Garden prior to the work reported here (see Figure 5). One, in 1992, consisted of archeological monitoring by Cox of trench excavation for the installation of buried utilities at three locations around the 
Acosta-Halff and Kampmann-Halff houses (41BX585 and 41BX586) at the north edge of the project area (Johnson and Cox 1995:10-11). In 2008, two trenches were excavated near the north edge of the project area in connection with the placement of a condensate line (Feit 2009). No intact archeological remains were found in either investigation.

\section{DISTURBANCE FACTORS}

At the outset of this project, it was recognized that most or all of Yanaguana Garden had been disturbed to some extent by demolition and land leveling for HemisFair '68, construction and subsequent removal of fair buildings, post-fair construction and landscaping, and placement of a variety of buried utilities. This was evident from the results of the archeological investigations described above, which showed ubiquitous disturbance to a depth of $1 \mathrm{ft}$ and common disturbance to $2-3 \mathrm{ft}$. It also was documented by analysis during an early stage of the project of the logs of 24 recent geotechnical boreholes placed throughout HemisFair Park. Two of these, in the far eastern part of the area near Interstate Highway 37, had extreme amounts of fill (10 and $13 \mathrm{ft}$ ), while 3 others, also in the eastern part, had no fill. Fill in the other 19, 12 of which were within or near Yanaguana Garden, ranged from 1.0 to $7.5 \mathrm{ft}$, averaging $2.7 \mathrm{ft}$.

The southwest corner of HemisFair Park did not suffer as much dramatic disturbance from the 1968 fair as did other parts of the park, such as areas impacted deeply by construction of water features and elevated ramps and large buildings such as the Tower of the Americas, convention center and theater, Texas Pavilion (now the Institute of Texan Cultures), U.S. Pavilion (now the John H. Wood Federal Courthouse and Adrian Spears Judicial Training Center), and Mexico Pavilion (now the Mexican Cultural Institute). But all of the area was cleared and leveled, except where historic buildings were left in place, and numerous temporary fair buildings were constructed (Figures 6-8). All or parts of seven buildings, covering 45,110 $\mathrm{ft}^{2}$, overlapped the project area. All of these were temporary buildings that probably had concrete slab foundations at or near grade (see Figure 6), but land modification for their erection, placement of utility lines to serve them, and their removal after 1968 certainly resulted in extensive disturbance.

The Yanaguana Garden construction plans showed numerous buried utilities crisscrossing the area. These included water lines, storm drains, communications lines, electrical lines, sewer lines, gas lines, and irrigation lines. As it turned out, many of these were inaccurately mapped, and many additional unmapped lines were found during archeological trenching and construction. Many of these lines were shallow ( $1 \mathrm{ft}$ or less) and thus were restricted to introduced fill, but others extended deeply into in situ deposits beneath the fill. Other disturbances obvious prior to the archeological work included construction of two parking lots (one between the Eager and Pereida houses and the other behind Beethoven Hall), a large playground feature, and various landscaping features such as tree wells, sidewalks, and curbs. 


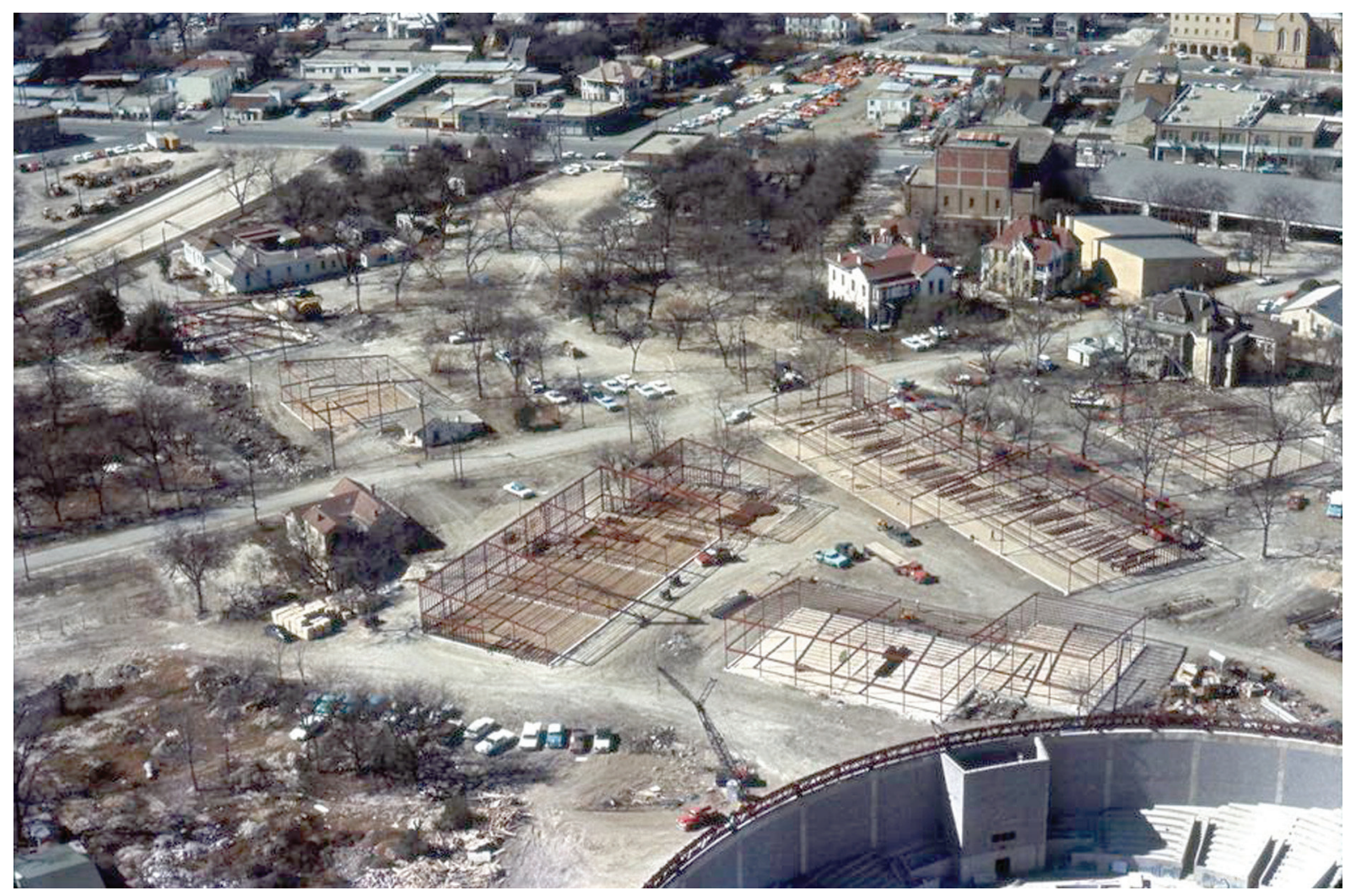

Figure 6. Aerial view to the southwest of the project area and adjoining areas showing the level of disturbance associated with construction of HemisFair '68. Intersection of South Alamo Street and East Cesar Chavez Boulevard (under construction) is at upper left, and Goliad Street runs left-right through the center. The rear of Beethoven Hall and adjacent Acosta-Halff and Kampmann-Halff houses are at upper right. Numerous temporary buildings and HemisFair Arena are under construction.

\section{OVERALL RECOMMENDATIONS}

The work done under the first four work orders for this contract allowed four main conclusions to be drawn about the archeological record of the Yanaguana Garden project area. First, almost all of the area was thoroughly disturbed to a depth of 2-5 ft or more as a result of filling of the Acequia Madre in the early twentieth century, demolition of buildings and land leveling in preparation for HemisFair '68, construction and subsequent demolition of temporary buildings for HemisFair '68, and landscape modifications made after removal of the temporary buildings. Second, this pervasive disturbance removed most or all traces of human activities performed on or near the original ground surface (including any remains relating to Native American occupation), with the only intact remains being the lower parts of historic features that extended well below that surface, such as the acequia ditch. Third, the lower parts of segments of the acequia are preserved in places at depths of 2-3 ft and more, but these segments are the stone-lined version that dates to the mid-nineteenth century or later; none of the documented segments contained intact deposits associated with use during the Spanish colonial era. Fourth, the vast majority of the archeological remains present relate to use during the latter part 


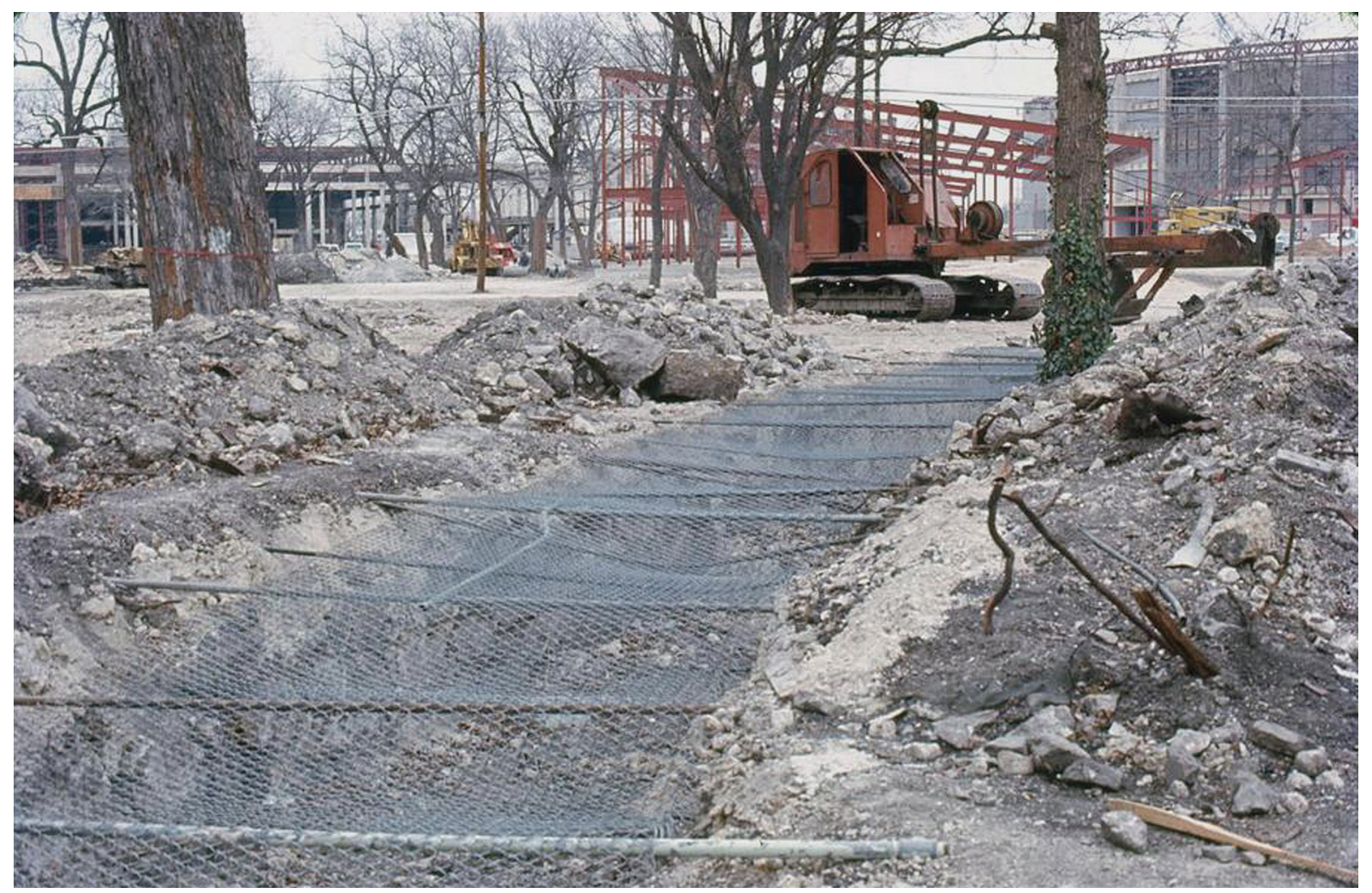

Figure 7. Ground-level view of disturbance associated with HemisFair '68 construction. Location and view direction are unknown, but the building on the upper right is the now-demolished HemisFair Arena, indicating that this is a generally northerly view in the Yanaguana Garden area. The trench may have been excavated to hold subsurface utilities. The metal-frame supports for a temporary building are visible behind the excavating equipment.

of the nineteenth century and the twentieth century; only a very small number of earlier Spanish colonial-era artifacts, and no intact features, had been documented, all in disturbed contexts.

These conclusions and review of the design plans led to two primary recommendations for treatment of archeological resources during development of Yanaguana Garden. First, where development would result in impacts extending deeper than 2-3 ft along the route of the acequia, development of a plan to avoid impacts or determine appropriate mitigation measures was recommended. Based on the plans, it appeared that the activities most likely to cause such disturbance were the following: (1) construction of the water play area, and in particular the splash pad; (2) removal of three trees in and adjacent to the water play area; (3) removal of an existing sanitary sewer line; (4) placement of two new sewer lines at depths of ca. $12 \mathrm{ft}$; (5) possible lowering of an existing gas line; (6) construction of a new emergency service drive that would cross the acequia alignment immediately south of its reconstructed segment; and (7) placement of some of the steel pipes to support the pergola above the south part of the promenade. Second, development of a plan to perform archeological monitoring where construction activities would extend 


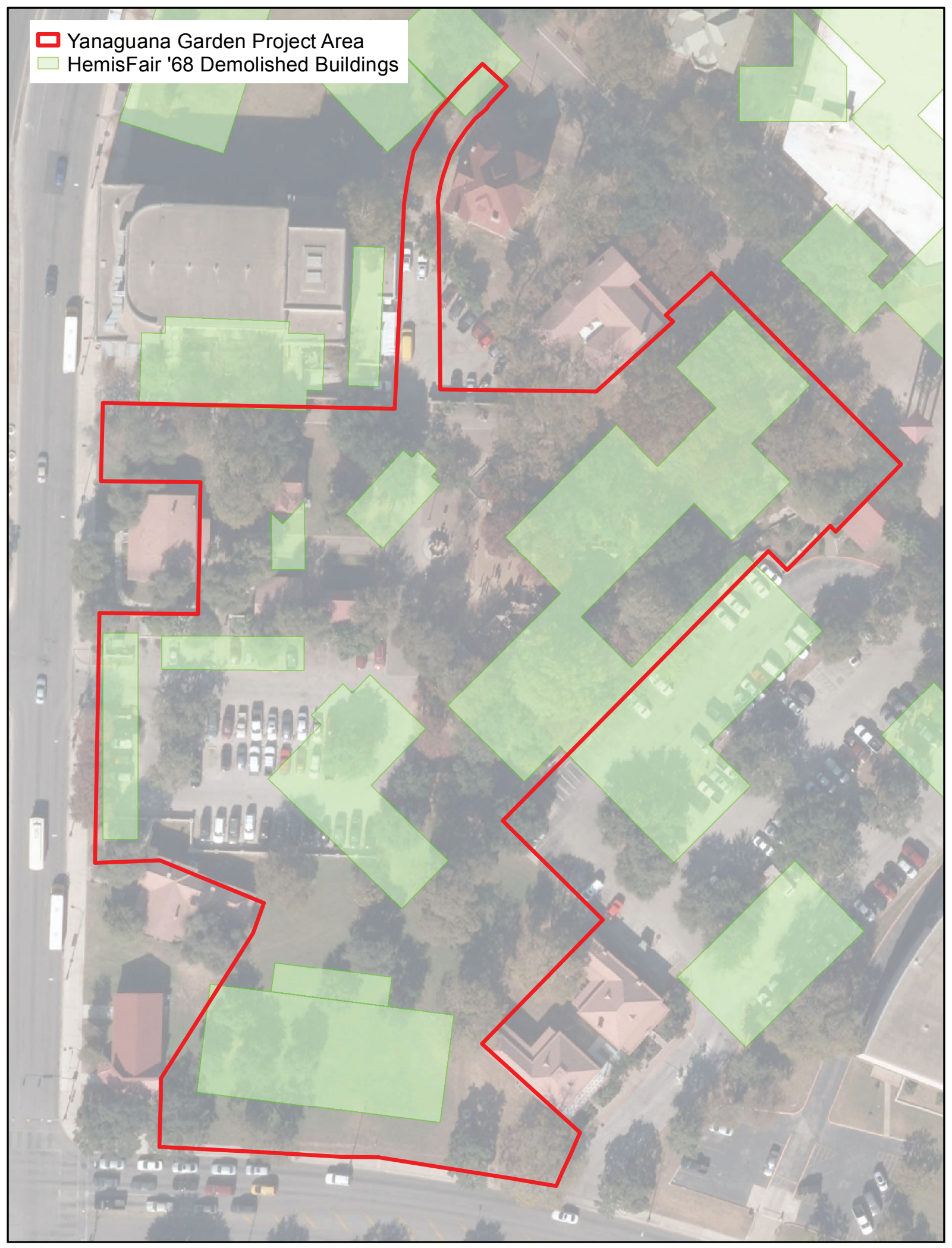

Figure 8. Plan showing locations of now-demolished HemisFair '68 temporary buildings in and adjacent to the Yanaguana Garden project area. 
to a depth of more than $2 \mathrm{ft}$ in parts of the project area other than the location of the acequia alignment was recommended to ensure that other intact deep features would not be inadvertently destroyed. The specifics of that plan would depend on the number, size, and spacing of deep impacts and their order in the construction sequence. The plans were not specific about where such deep impacts could occur, but likely places included the west-central part where new bathrooms would be constructed, the part of the water play area outside the acequia corridor, the sand play area, and the areas of three bioswales (one in the north part and two in the south) that would serve as drains for water runoff.

Subsequent to presentation of these recommendations, CoSA-TCI, in consultation with THC, determined that full-time archeological monitoring of all demolition and construction at Yanaguana Garden was warranted. This determination led to the sixth work order. 



\section{CHAPTER 3: WORK ACCOMPLISHED}

The archeological file search, literature review, and data compilation began during the 2012 planning project and continued under two subsequent work orders that are part of the project reported here. These tasks had two primary objectives: (1) to identify known and as-yet-undiscovered sites that could be, or that could have once been, in the Yanaguana Garden project area; and (2) to assess the likelihood that intact portions of such sites are still present, given the amount of disturbance the area has seen. A variety of sources were consulted to accomplish this, with the most important ones being the Texas Historical Commission's Archeological Sites Atlas; reports on previous archeological investigations at HemisFair Park; an 1835 map entitled "San Antonio and Its Environs" (Foster et al. 2006); the 1873 and 1886 Augustus Koch bird's-eye views of San Antonio (Koch 1873, 1886); an 1883 map entitled "Morrison and Fourmy's Revised Map of City of San Antonio Texas" (Gast 1883); an 1889 City of San Antonio map (Olsen 1889); Sanborn maps dating to 1892, 1896, and 1912 (Sanborn Fire Insurance Company 1892, 1896, 1912); a 1939 aerial photograph (Tobin International, Ltd. 1939); the 1953 San Antonio East USGS quadrangle; maps of pre-HemisFair buildings presented by Dupont et al. (2011) in the UTSA Center for Cultural Sustainability's inventory of historic resources at HemisFair Park; maps showing the locations of HemisFair '68 temporary buildings; photographs taken during construction of HemisFair '68 obtained from the University of North Texas Libraries, Portal to Texas History; reports on recent geotechnical investigations at HemisFair Park; and the Yanaguana Garden construction plans.

Fieldwork at Yanaguana Garden was conducted under four work orders, starting on May 1 and ending on December 24, 2014. These are described below.

\section{WORK ORDER 37-2014-04-24}

This first work order consisted of one day of archeological trenching by two archeologists where a new storm drain was planned (May 1), followed by a half day of monitoring by one archeologist of excavation for placement of an adjacent storm drain junction box (June 11), at the location of a new emergency service drive. This was at the far north edge of the project area, immediately north and west of the Acosta-Halff house (41BX585; Figure 9). Archeological attention to the storm drain component of this project was considered warranted because this was the only area that would see deep disturbance. 


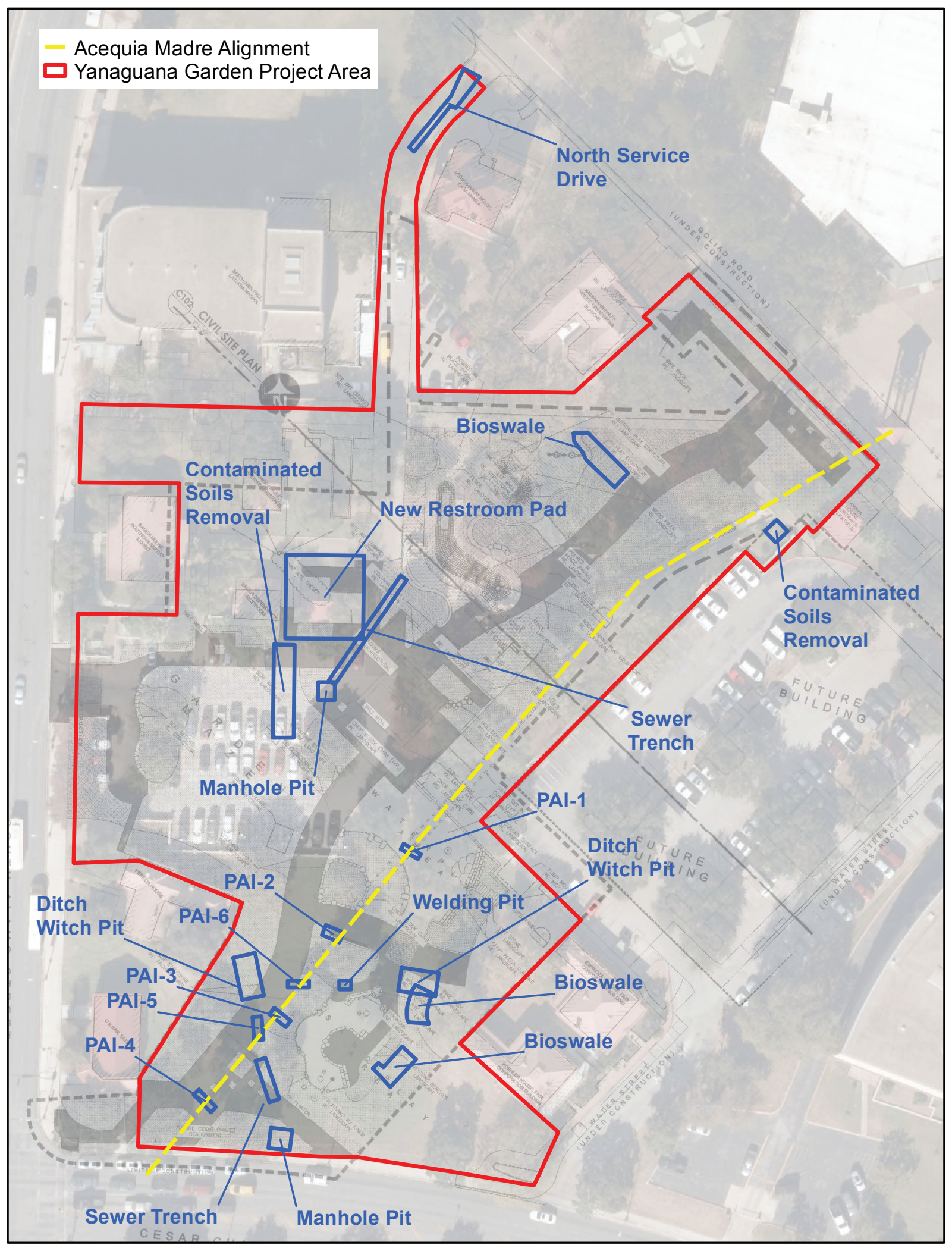

Figure 9. Plan of the project area showing areas investigated archeologically. Site locations are not shown in report copies for public distribution. 
The single trench excavated before construction began was $12 \mathrm{~m}(39 \mathrm{ft})$ long and typically was $1.5 \mathrm{~m}$ (5 ft) deep. No archeological features were observed. The full length of the trench contained disturbed construction fill (rock and concrete rubble, as well as finer-grained deposits) to depths of $0.6-1.5 \mathrm{~m}(2-5 \mathrm{ft})$ below the surface. Beneath the construction fill in parts of the trench were in situ A and B soil horizons on Cretaceous-age bedrock. A few historic-age artifacts (a transferware ceramic sherd, a patinated bottle glass neck, and a horseshoe) were observed within the disturbed fill, but they clearly did not represent in situ archeological deposits. The storm drain junction box excavation that was monitored measured $6.2 \mathrm{~m}(20 \mathrm{ft})$ northeast-southwest by 1.7-3.4 m (6-11 ft) northwest-southeast; it overlapped the north end of the archeological trench and extended a short distance northeast of

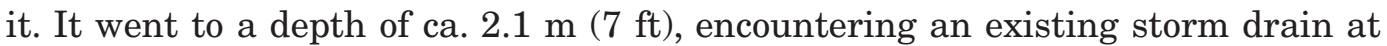
$1.2-1.8 \mathrm{~m}(4-6 \mathrm{ft})$. The walls of this excavation showed $0.5-0.9 \mathrm{~m}(1.5-3.0 \mathrm{ft})$ of brown fine-grained and coarse rubble fill over in situ sediments. The lower part of the fill zone in the southeast wall had numerous jumbled yellow bricks like those used in the Acosta-Halff House just to the south, suggesting that debris from house construction had been placed here. No other historic materials and no historic features were observed.

\section{WORK ORDER 39-2014-07-24}

Monitoring was done over two-and-a-half days (September 29-30 and October 2) in connection with removal of contaminated soils in two areas (see Figure 9). One (AOC-1) was a $65 \times 10$-ft area beneath the parking lot south of the Eager House and Dependency (41BX587), and the other (AOC-2) was a 7x7-ft area immediately east of the north end of the reconstructed segment of the Acequia Madre (41BX8). Sediments were removed to depths of $2.5 \mathrm{ft}$ in AOC-1 and $5 \mathrm{ft}$ in AOC-2. An archeologist observed excavation of these areas by a mini-excavator and placement of the materials removed in the bucket of a skid-steer loader for transport to a roll-off box.

The AOC-1 excavation encountered, in addition to numerous buried utility lines, 1.0-1.5 ft of asphalt and gravelly construction base material across the full area. Below this to $2.5 \mathrm{ft}$ in the southern third of the AOC was construction debris consisting of gravels, concrete chunks, rebar, and churned natural black clayey sediments. The northern third contained mostly filled utility trenches with some areas of churned natural black clay. In the middle third of the AOC, black clay directly underlay the asphalt and gravelly parking lot base, but modern debris within it, including large concrete chunks in the bottom of the excavation, indicated that these natural sediments had been thoroughly disturbed, probably during land leveling for construction of HemisFair '68 and later during removal of HemisFair '68 temporary buildings and parking lot construction. Materials observed in the excavation, in addition to abundant concrete and rebar, included lumber, displaced wood posts, asphalt shingles, machine-made bricks, sewer pipe, ceramic tiles, glass, metal, stove pipe flashing, angle iron, galvanized metal pipe, iron pipe, plastic, and cloth fabric. A single diagnostic bottle was observed; it was a clear glass Sanford's ink bottle manufactured in 1939 . 
The AOC-2 excavation was 2.5-3.5 ft east of the north end of the reconstructed Acequia Madre wall. The general stratigraphy consisted of the following: 0-8 inches, landscape fill; 8-24/34 inches, gravelly fill with sandstone chunks, with the bottom sloping down to the west toward the acequia; 24/34-36/48 inches, black clay with limestone and gravel inclusions (churned natural sediment); 36/48-55 inches, black clay without obvious limestone and gravel inclusions (in situ natural sediment?); 55-60+ inches, limestone bedrock. Hence, this area had 2-3 ft of fill over ca. $1 \mathrm{ft}$ of disturbed natural sediments. No archeological features were observed. Artifacts noted included shaped limestone blocks (maybe from original acequia construction and 1968 reconstruction), animal bones, a metal spike, other metal, glass (clear, amber, green, and aquamarine), plastic, and concrete rubble. Some of the glass was patinated and could date to the late-nineteenth or early-twentieth century but was not otherwise distinctive. These materials were consistent with the kinds of remains that Schuetz (1970) found in excavating the acequia nearby, which largely reflected 1890s-1930s trash disposal.

\section{WORK ORDER 43-2014-08-04}

Two archeologists performed archeological trenching in the south part of the project area over two-and-a-half days (August 20-22) to determine if intact portions of the Acequia Madre (41BX8) are present there. The area investigated extended for a distance of about $60 \mathrm{~m}(200 \mathrm{ft})$, starting ca. $20 \mathrm{~m}(66 \mathrm{ft})$ southwest of the end of the reconstructed segment of the acequia and ending just north of East Cesar Chavez Boulevard (see Figure 9). The four trenches excavated were (PAI 1-4) 22, 48,60 , and $79 \mathrm{~m}(72,157,197$, and $259 \mathrm{ft})$ southwest of the end of the reconstructed segment of the acequia. They were 5.4-5.8 m (17.7-19.0 ft) long, 1.0-2.0 m (3-6 ft) wide, and 1.8-2.5 m (5.9-8.2 ft) deep. All four were oriented northwest-southeast, perpendicular to the route of the acequia.

Details on what was found are included below under Results of Archeological Investigations; the paragraphs here present a summary. Two of the four trenches (PAI-1 and PAI-4) found preserved segments of the rock wall that was built in the mid 1800s to line the east wall of the acequia (Fox and Cox 1990:1). In both cases, the top of what is left of the wall was $0.9-1.0 \mathrm{~m}(3.0-3.3 \mathrm{ft})$ below the modern surface, with the overlying deposits consisting of disturbed fill. Hence, it appears that the preserved segments are the basal portions of the original wall, with the upper 0.9-1.0 m (3.0-3.3 ft) having been removed during land clearing, building construction, and building demolition before, during, and after HemisFair' 68 . A third trench (PAI-3) found what may be the very bottom of the acequia ditch but no intact rock walls. Schuetz's (1970) excavations to the north showed that the acequia was $1.9 \mathrm{~m}(6.2 \mathrm{ft})$ wide, but no traces of the west wall were found in the current project area, which is consistent with the scant evidence for a west wall in the excavations done in this area by Fox (1985) and Fox and Cox (1990). According to Fox and Cox (1990:22), a 1915 map noting "no well defined ditch line" where the west wall would have been suggests that it had been removed by that time.

Schuetz's (1970) excavations showed that the acequia ditch extended $1.6 \mathrm{~m}$ (5.2 ft) below the surface, and Trenches PAI-1 and PAI-4 encountered ditch fill 
extending to the same depth in those locations. Possible ditch fill extended deeper (6.6 ft) in Trench PAI-3, suggesting more modern fill there than elsewhere. None of the trenches found anything that could be related to the 1720s-mid-1800s version of the acequia that existed before it was lined with rock walls, and it is possible that most or all traces of that ditch were obliterated when the walls were built. Likewise, no artifacts associated with early use of the feature were found; all materials observed related to trash discard and intentional ditch filling in the latenineteenth and early-mid-twentieth centuries, or to more-recent accumulations of debris relating to construction and demolition of HemisFair '68 buildings.

\section{WORK ORDER 46-2014-10-06}

A single archeologist performed archeological construction monitoring on 46 work days, starting on October 13 and ending on December 24 (Table 2). The archeologist was present whenever ground-disturbing activities occurred, and frequently when no such activities were under way. Multiple contractors performed the various jobs associated with removal of old improvements and preparation for installation of new ones, employing various pieces of large equipment for demolition, loading, hauling, excavating, and trenching. The archeologist remained nearby when subsurface impacts occurred, taking photographs and notes to record the nature of the subsurface deposits as they were revealed. In addition, whenever construction crew members noticed potential archeological remains, they notified the archeologist, who then examined and evaluated them.

Monitoring was done of various activities that resulted in both shallow and deep subsurface impacts. Shallow impacts are defined as those that reached no more than ca. $2 \mathrm{ft}$ deep and typically were confined to the introduced caliche base or construction fill that is common to nearly all of the project area. Deep subsurface impacts are those that reached beyond $2 \mathrm{ft}$, usually extending through the layers of introduced fill into in situ sediments and sometimes the underlying Austin Chalk bedrock. The deepest impacts reached $13 \mathrm{ft}$ below the ground surface. Many activities, with both shallow and deep impacts, occurred multiple times throughout the course of monitoring, with multiple disturbances often occurring on any given day.

\section{Monitoring of Shallow Impacts}

Shallow subsurface impacts were frequent and pervasive across the project area (and hence their locations are not shown on Figure 9). Discussed here are some of the more-common examples.

Playscape and Gazebo Demolition: The former playscape and gazebo were demolished early in the project (Figure 10a). Much of the work occurred above ground and required little monitoring; however, monitoring did occur when corner posts and uprights were removed from the ground. Some of these posts extended beyond $2 \mathrm{ft}$ deep, but they were confined to introduced fill nonetheless.

Masonry Wall Demolition: Masonry walls enclosed multiple areas around the park. Each of these walls had to be removed and was knocked over using a large 
Table 2. Daily log of archeological construction monitoring activities under Work Order 46-2014-10-66

\begin{tabular}{|c|c|c|c|c|c|}
\hline Date & Activities Monitored & $\begin{array}{l}\text { Location of } \\
\text { Monitoring }\end{array}$ & $\begin{array}{l}\text { Depth of } \\
\text { Impacts }\end{array}$ & $\begin{array}{l}\text { Nature of } \\
\text { Deposits }\end{array}$ & $\begin{array}{l}\text { Archeological } \\
\text { Remains }\end{array}$ \\
\hline \multirow[t]{3}{*}{ 13-Oct 14} & playscape demolition & west of acequia & ca. $3 \mathrm{ft}$ & $\begin{array}{l}\text { construction } \\
\text { fill }\end{array}$ & none \\
\hline & gazebo demolition & gazebo location & surface & $\begin{array}{l}\text { construction } \\
\text { fill }\end{array}$ & none \\
\hline & $\begin{array}{l}\text { concrete wall } \\
\text { demolition }\end{array}$ & $\begin{array}{l}\text { east edge of parking } \\
\text { lot }\end{array}$ & ca. $3 \mathrm{ft}$ & $\begin{array}{l}\text { construction } \\
\text { fill }\end{array}$ & none \\
\hline $14-$ Oct 14 & $\begin{array}{l}\text { playscape pier } \\
\text { removal }\end{array}$ & $\begin{array}{l}\text { north end of } \\
\text { playscape }\end{array}$ & ca. $3 \mathrm{ft}$ & $\begin{array}{l}\text { construction } \\
\text { fill }\end{array}$ & none \\
\hline \multirow[t]{3}{*}{ 15-Oct 14} & $\begin{array}{l}\text { playscape concrete } \\
\text { removal }\end{array}$ & $\begin{array}{l}\text { north end of } \\
\text { playscape }\end{array}$ & ca. $1 \mathrm{ft}$ & $\begin{array}{l}\text { construction } \\
\text { fill }\end{array}$ & ceramic sewer pipe \\
\hline & brick paver removal & north of playscape & $0.5 \mathrm{ft}$ & $\begin{array}{l}\text { construction } \\
\text { fill }\end{array}$ & none \\
\hline & tree removal & north of playscape & $3 \mathrm{ft}$ & $\begin{array}{l}\text { construction } \\
\text { fill }\end{array}$ & $\begin{array}{l}\text { glass; pre-1903 } \\
\text { bottle neck }\end{array}$ \\
\hline \multirow[t]{3}{*}{ 16-Oct 14} & $\begin{array}{l}\text { pavement removal for } \\
\text { construction entrance }\end{array}$ & $\begin{array}{l}\text { construction entrance } \\
\text { off Alamo St. }\end{array}$ & $<1 \mathrm{ft}$ & $\begin{array}{l}\text { construction } \\
\text { fill }\end{array}$ & none \\
\hline & brick paver removal & north of playscape & $0.5 \mathrm{ft}$ & $\begin{array}{l}\text { construction } \\
\text { fill }\end{array}$ & none \\
\hline & tree removal & $\begin{array}{l}\text { around playscape } \\
\text { location }\end{array}$ & $1-2 \mathrm{ft}$ & $\begin{array}{l}\text { construction } \\
\text { fill }\end{array}$ & none \\
\hline \multirow[t]{2}{*}{ 17-Oct 14} & brick paver removal & $\begin{array}{l}\text { northeast corner of } \\
\text { project area }\end{array}$ & $0.5 \mathrm{ft}$ & $\begin{array}{l}\text { construction } \\
\text { fill }\end{array}$ & none \\
\hline & shrub removal & $\begin{array}{l}\text { northeast corner of } \\
\text { project area }\end{array}$ & $1 \mathrm{ft}$ & $\begin{array}{l}\text { construction } \\
\text { fill }\end{array}$ & none \\
\hline $20-$ Oct 14 & tree removal & $\begin{array}{l}\text { around parking lot; } \\
\text { south of acequia; } \\
\text { around women's } \\
\text { restroom }\end{array}$ & $1-3.5 \mathrm{ft}$ & $\begin{array}{l}\text { construction } \\
\text { fill; original } \\
\text { surface }\end{array}$ & $\begin{array}{l}\text { cut nails; glass; } \\
\text { ceramic insulator; } \\
\text { stoneware fragment; } \\
\text { child's tea set saucer } \\
\text { fragment (historic } \\
\text { trash scatter) }\end{array}$ \\
\hline \multirow[t]{2}{*}{$21-$ Oct 14} & rubble/debris hauling & N/A & N/A & N/A & N/A \\
\hline & $\begin{array}{l}\text { concrete wall } \\
\text { demolition }\end{array}$ & $\begin{array}{l}\text { near women's } \\
\text { restroom }\end{array}$ & $1-4 \mathrm{ft}$ & $\begin{array}{l}\text { construction } \\
\text { fill }\end{array}$ & none \\
\hline \multirow[t]{2}{*}{$22-$ Oct 14} & concrete curb removal & $\begin{array}{l}\text { east edge of parking } \\
\text { lot }\end{array}$ & $1-2 \mathrm{ft}$ & $\begin{array}{l}\text { construction } \\
\text { fill }\end{array}$ & none \\
\hline & $\begin{array}{l}\text { light pole pedestal } \\
\text { removal }\end{array}$ & $\begin{array}{l}\text { throughout project } \\
\text { area }\end{array}$ & $3 \mathrm{ft}$ & $\begin{array}{l}\text { construction } \\
\text { fill }\end{array}$ & iron gear \\
\hline \multirow[t]{4}{*}{$23-$ Oct 14} & $\begin{array}{l}\text { concrete wall } \\
\text { demolition }\end{array}$ & $\begin{array}{l}\text { south end of parking } \\
\text { lot }\end{array}$ & $1-2 \mathrm{ft}$ & $\begin{array}{l}\text { construction } \\
\text { fill }\end{array}$ & none \\
\hline & rubble/debris hauling & N/A & N/A & N/A & N/A \\
\hline & $\begin{array}{l}\text { concrete wall } \\
\text { demolition }\end{array}$ & women's restroom & $2-4 \mathrm{ft}$ & $\begin{array}{l}\text { construction } \\
\text { fill }\end{array}$ & none \\
\hline & $\begin{array}{l}\text { playscape pier } \\
\text { removal }\end{array}$ & playscape area & $2 \mathrm{ft}$ & $\begin{array}{l}\text { construction } \\
\text { fill }\end{array}$ & none \\
\hline \multirow[t]{5}{*}{ 24-Oct 14} & $\begin{array}{l}\text { concrete wall } \\
\text { demolition }\end{array}$ & women's restroom & $3-4 \mathrm{ft}$ & $\begin{array}{l}\text { construction } \\
\text { fill }\end{array}$ & none \\
\hline & concrete curb removal & parking lot & $1-2 \mathrm{ft}$ & $\begin{array}{l}\text { construction } \\
\text { fill }\end{array}$ & none \\
\hline & rubble/debris hauling & N/A & N/A & N/A & N/A \\
\hline & concrete curb removal & along acequia & $1-2 \mathrm{ft}$ & $\begin{array}{l}\text { construction } \\
\text { fill }\end{array}$ & none \\
\hline & $\begin{array}{l}\text { light pole pedestal } \\
\text { removal }\end{array}$ & $\begin{array}{l}\text { north end of } \\
\text { playscape }\end{array}$ & $3-5 \mathrm{ft}$ & $\begin{array}{l}\text { construction } \\
\text { fill }\end{array}$ & none \\
\hline
\end{tabular}


Table 2, continued

\begin{tabular}{|c|c|c|c|c|c|}
\hline Date & Activities Monitored & $\begin{array}{l}\text { Location of } \\
\text { Monitoring }\end{array}$ & $\begin{array}{l}\text { Depth of } \\
\text { Impacts }\end{array}$ & $\begin{array}{l}\text { Nature of } \\
\text { Deposits }\end{array}$ & $\begin{array}{c}\text { Archeological } \\
\text { Remains } \\
\end{array}$ \\
\hline \multirow[t]{3}{*}{$27-$ Oct 14} & concrete curb removal & $\begin{array}{l}\text { north end of } \\
\text { playscape }\end{array}$ & $1-2 \mathrm{ft}$ & $\begin{array}{l}\text { construction } \\
\text { fill } \\
\end{array}$ & none \\
\hline & concrete curb removal & $\begin{array}{l}\text { east of Carriage } \\
\text { house }\end{array}$ & $1-2 \mathrm{ft}$ & $\begin{array}{l}\text { natural } \\
\text { surface }\end{array}$ & $\begin{array}{l}\text { glass; chisel; hinge; } \\
\text { brick; wire nails; cut } \\
\text { bone (historic trash } \\
\text { scatter) }\end{array}$ \\
\hline & tree removal & $\begin{array}{l}\text { north end of } \\
\text { playscape }\end{array}$ & $3 \mathrm{ft}$ & $\begin{array}{l}\text { construction } \\
\text { fill }\end{array}$ & none \\
\hline \multirow[t]{3}{*}{ 28-Oct 14} & concrete curb removal & $\begin{array}{l}\text { north end of } \\
\text { playscape }\end{array}$ & $1-2 \mathrm{ft}$ & $\begin{array}{l}\text { construction } \\
\text { fill } \\
\end{array}$ & none \\
\hline & concrete curb removal & $\begin{array}{l}\text { east of Carriage } \\
\text { house }\end{array}$ & $1-2 \mathrm{ft}$ & $\begin{array}{l}\text { natural } \\
\text { surface }\end{array}$ & $\begin{array}{l}\text { marble; cut bone } \\
\text { (historic trash } \\
\text { scatter) }\end{array}$ \\
\hline & $\begin{array}{l}\text { restroom service line } \\
\text { locate }\end{array}$ & women's restroom & $4 \mathrm{ft}$ & $\begin{array}{l}\text { construction } \\
\text { fill } \\
\end{array}$ & none \\
\hline \multirow[t]{3}{*}{$29-$ Oct 14} & pavement remvoal & parking lot & $0.5 \mathrm{ft}$ & $\begin{array}{l}\text { construction } \\
\text { fill } \\
\end{array}$ & none \\
\hline & $\begin{array}{l}\text { pipe and wire } \\
\text { removal }\end{array}$ & $\begin{array}{l}\text { north end of service } \\
\text { drive }\end{array}$ & $1-1.5 \mathrm{ft}$ & $\begin{array}{l}\text { construction } \\
\text { fill } \\
\end{array}$ & none \\
\hline & concrete curb removal & parking lot & $1-2 \mathrm{ft}$ & $\begin{array}{l}\text { construction } \\
\text { fill }\end{array}$ & none \\
\hline \multirow[t]{3}{*}{ 30-Oct 14} & pavement removal & parking lot & $0.5 \mathrm{ft}$ & $\begin{array}{l}\text { construction } \\
\text { fill }\end{array}$ & none \\
\hline & concrete curb removal & parking lot & $1-2 \mathrm{ft}$ & $\begin{array}{l}\text { construction } \\
\text { fill } \\
\end{array}$ & none \\
\hline & rubble/debris hauling & N/A & N/A & N/A & N/A \\
\hline \multirow[t]{2}{*}{ 31-Oct 14} & $\begin{array}{l}\text { concrete wall } \\
\text { demolition }\end{array}$ & along Alamo St. & $1-2 \mathrm{ft}$ & $\begin{array}{l}\text { construction } \\
\text { fill } \\
\end{array}$ & none \\
\hline & $\begin{array}{l}\text { women's restroom } \\
\text { demolition }\end{array}$ & women's restroom & $\begin{array}{l}\text { above } \\
\text { ground }\end{array}$ & N/A & none \\
\hline \multirow[t]{4}{*}{ 3-Nov 14} & $\begin{array}{l}\text { women's restroom } \\
\text { demolition } \\
\end{array}$ & women's restroom & $1-2 \mathrm{ft}$ & $\begin{array}{l}\text { construction } \\
\text { fill }\end{array}$ & none \\
\hline & rubble/debris hauling & N/A & N/A & N/A & N/A \\
\hline & brick paver removal & women's restroom & $1-2 \mathrm{ft}$ & $\begin{array}{l}\text { construction } \\
\text { fill } \\
\end{array}$ & none \\
\hline & concrete curb removal & women's restroom & $1-2 \mathrm{ft}$ & $\begin{array}{l}\text { construction } \\
\text { fill }\end{array}$ & none \\
\hline \multirow[t]{2}{*}{ 4-Nov 14} & leveling of materials & women's restroom & $1-2 \mathrm{ft}$ & $\begin{array}{l}\text { construction } \\
\text { fill }\end{array}$ & none \\
\hline & potholing for utilities & pump house location & $3-4 \mathrm{ft}$ & $\begin{array}{l}\text { construction } \\
\text { fill; original } \\
\text { surface }\end{array}$ & $\begin{array}{l}\text { cut and wire nails; } \\
\text { ceramics; glass; } \\
\text { marble (historic } \\
\text { trash scatter) }\end{array}$ \\
\hline \multirow[t]{2}{*}{ 10-Nov 14} & site grading & $\begin{array}{l}\text { north end of project } \\
\text { area }\end{array}$ & $1 \mathrm{ft}$ & $\begin{array}{l}\text { construction } \\
\text { fill }\end{array}$ & $\begin{array}{l}\text { fragment of flow } \\
\text { blue found in loose } \\
\text { dirt }\end{array}$ \\
\hline & staking & $\begin{array}{l}\text { north end of project } \\
\text { area }\end{array}$ & N/A & $\begin{array}{l}\text { construction } \\
\text { fill }\end{array}$ & none \\
\hline \multirow[t]{2}{*}{ 11-Nov 14} & $\begin{array}{l}\text { excavation of } \\
\text { bioswale pit }\end{array}$ & $\begin{array}{l}\text { north end of project } \\
\text { area }\end{array}$ & $5 \mathrm{ft}$ & $\begin{array}{l}\text { construction } \\
\text { fill; original } \\
\text { surface }\end{array}$ & $\begin{array}{l}\text { glass, bricks, sewer } \\
\text { pipe (mid- } 20 \text { th- } \\
\text { century trash); brick } \\
\text { pier base with post } \\
\text { inside }\end{array}$ \\
\hline & site grading & $\begin{array}{l}\text { north end of project } \\
\text { area }\end{array}$ & $1 \mathrm{ft}$ & $\begin{array}{l}\text { construction } \\
\text { fill }\end{array}$ & none \\
\hline
\end{tabular}


Table 2, continued

\begin{tabular}{|c|c|c|c|c|c|}
\hline Date & Activities Monitored & $\begin{array}{l}\text { Location of } \\
\text { Monitoring }\end{array}$ & $\begin{array}{l}\text { Depth of } \\
\text { Impacts }\end{array}$ & $\begin{array}{l}\text { Nature of } \\
\text { Deposits }\end{array}$ & $\begin{array}{c}\text { Archeological } \\
\text { Remains } \\
\end{array}$ \\
\hline \multirow[t]{2}{*}{ 12-Nov 14} & $\begin{array}{l}\text { trenching for water } \\
\text { line }\end{array}$ & near restroom pad & $4-5 \mathrm{ft}$ & $\begin{array}{l}\text { construction } \\
\text { fill } \\
\end{array}$ & none \\
\hline & site grading & $\begin{array}{l}\text { fire lane and old } \\
\text { parking lot }\end{array}$ & $1 \mathrm{ft}$ & $\begin{array}{l}\text { construction } \\
\text { fill } \\
\end{array}$ & none \\
\hline \multirow[t]{2}{*}{ 17-Nov 14} & site grading & old parking lot & $<1 \mathrm{ft}$ & $\begin{array}{l}\text { construction } \\
\text { fill }\end{array}$ & none \\
\hline & $\begin{array}{l}\text { excavation of } \\
\text { restroom pad }\end{array}$ & site of restroom & $3.5 \mathrm{ft}$ & $\begin{array}{l}\text { construction } \\
\text { fill; original } \\
\text { surface } \\
\end{array}$ & none \\
\hline \multirow[t]{5}{*}{ 18-Nov 14} & $\begin{array}{l}\text { excavation of } \\
\text { restroom pad }\end{array}$ & site of restroom & $3.5 \mathrm{ft}$ & $\begin{array}{l}\text { construction } \\
\text { fill; original } \\
\text { surface } \\
\end{array}$ & $\begin{array}{l}\text { small glass } \\
\text { fragments }\end{array}$ \\
\hline & brick paver removal & fire lane & $<0.5 \mathrm{ft}$ & $\begin{array}{l}\text { construction } \\
\text { fill } \\
\end{array}$ & none \\
\hline & utility searching & $\begin{array}{l}\text { throughout project } \\
\text { area }\end{array}$ & $1-3 \mathrm{ft}$ & $\begin{array}{l}\text { construction } \\
\text { fill } \\
\end{array}$ & none \\
\hline & $\begin{array}{l}\text { trenching for water } \\
\text { line }\end{array}$ & around restroom pad & $1-2 \mathrm{ft}$ & $\begin{array}{l}\text { construction } \\
\text { fill }\end{array}$ & none \\
\hline & site grading & $\begin{array}{l}\text { throughout project } \\
\text { area }\end{array}$ & $<1 \mathrm{ft}$ & $\begin{array}{l}\text { construction } \\
\text { fill }\end{array}$ & none \\
\hline \multirow[t]{4}{*}{ 19-Nov 14} & loading of spoil & N/A & N/A & N/A & N/A \\
\hline & site grading & southern project area & $1 \mathrm{ft}$ & $\begin{array}{l}\text { construction } \\
\text { fill } \\
\end{array}$ & none \\
\hline & $\begin{array}{l}\text { trenching for water } \\
\text { line }\end{array}$ & site of restroom & $1-2 \mathrm{ft}$ & $\begin{array}{l}\text { construction } \\
\text { fill } \\
\end{array}$ & none \\
\hline & $\begin{array}{l}\text { potholing for utilities } \\
\text { (vacuum truck) }\end{array}$ & pump house area & $4-5 \mathrm{ft}$ & $\begin{array}{l}\text { construction } \\
\text { fill; original } \\
\text { surface } \\
\end{array}$ & none \\
\hline \multirow[t]{4}{*}{ 20-Nov 14} & site grading & southern project area & $1 \mathrm{ft}$ & $\begin{array}{l}\text { construction } \\
\text { fill }\end{array}$ & none \\
\hline & $\begin{array}{l}\text { utility searching } \\
\text { (vacuum truck) }\end{array}$ & southern project area & $1-10 \mathrm{ft}$ & $\begin{array}{l}\text { construction } \\
\text { fill; original } \\
\text { surface } \\
\end{array}$ & glass \\
\hline & loading of spoil & N/A & N/A & N/A & N/A \\
\hline & $\begin{array}{l}\text { trenching for sewer } \\
\text { line }\end{array}$ & $\begin{array}{l}\text { southern project } \\
\text { area, across acequia } \\
\text { alignment }\end{array}$ & $5-6 \mathrm{ft}$ & $\begin{array}{l}\text { construction } \\
\text { fill; original } \\
\text { surface }\end{array}$ & $\begin{array}{l}\text { glass, ceramics, axe } \\
\text { head (historic trash } \\
\text { scatter); no evidence } \\
\text { of acequia }\end{array}$ \\
\hline \multirow[t]{3}{*}{ 21-Nov 14} & loading of spoil & N/A & N/A & N/A & N/A \\
\hline & $\begin{array}{l}\text { leveling of restroom } \\
\text { pad }\end{array}$ & N/A & N/A & N/A & N/A \\
\hline & $\begin{array}{l}\text { utility searching } \\
\text { (vacuum truck) }\end{array}$ & southern project area & $3-4 \mathrm{ft}$ & $\begin{array}{l}\text { construction } \\
\text { fill; original } \\
\text { surface } \\
\end{array}$ & none \\
\hline \multirow[t]{3}{*}{ 24-Nov 14} & $\begin{array}{l}\text { excavation of } \\
\text { bioswale pit }\end{array}$ & southern project area & $5 \mathrm{ft}$ & $\begin{array}{l}\text { construction } \\
\text { fill; original } \\
\text { surface } \\
\end{array}$ & $\begin{array}{l}\text { few glass fragments, } \\
\text { sewer pipe }\end{array}$ \\
\hline & site grading & southern project area & $1 \mathrm{ft}$ & $\begin{array}{l}\text { construction } \\
\text { fill } \\
\end{array}$ & none \\
\hline & utility searching & southern project area & $1 \mathrm{ft}$ & $\begin{array}{l}\text { construction } \\
\text { fill } \\
\end{array}$ & none \\
\hline \multirow[t]{2}{*}{ 25-Nov 14} & $\begin{array}{l}\text { leveling of restroom } \\
\text { pad }\end{array}$ & N/A & N/A & N/A & N/A \\
\hline & rubble/debris hauling & N/A & N/A & N/A & N/A \\
\hline
\end{tabular}


Table 2, continued

\begin{tabular}{|c|c|c|c|c|c|}
\hline Date & Activities Monitored & $\begin{array}{l}\text { Location of } \\
\text { Monitoring }\end{array}$ & $\begin{array}{l}\text { Depth of } \\
\text { Impacts }\end{array}$ & $\begin{array}{l}\text { Nature of } \\
\text { Deposits }\end{array}$ & $\begin{array}{c}\text { Archeological } \\
\text { Remains }\end{array}$ \\
\hline 26-Nov 14 & $\begin{array}{l}\text { no ground } \\
\text { disturbance }\end{array}$ & N/A & N/A & N/A & N/A \\
\hline \multirow[t]{2}{*}{ 1-Dec 14} & $\begin{array}{l}\text { demolition of Solis } \\
\text { house }\end{array}$ & Solis house & N/A & N/A & N/A \\
\hline & $\begin{array}{l}\text { leveling of restroom } \\
\text { pad }\end{array}$ & N/A & N/A & N/A & N/A \\
\hline \multirow[t]{2}{*}{ 2-Dec 14} & $\begin{array}{l}\text { demolition of Solis } \\
\text { house }\end{array}$ & Solis house & $1 \mathrm{ft}$ & $\begin{array}{l}\text { construction } \\
\text { fill } \\
\end{array}$ & N/A \\
\hline & site grading & southern project area & $1 \mathrm{ft}$ & $\begin{array}{l}\text { construction } \\
\text { fill }\end{array}$ & none \\
\hline \multirow[t]{3}{*}{ 3-Dec 14} & site grading & southern project area & $1 \mathrm{ft}$ & $\begin{array}{l}\text { construction } \\
\text { fill }\end{array}$ & none \\
\hline & $\begin{array}{l}\text { excavation of } \\
\text { bioswale pit }\end{array}$ & southern project area & $5 \mathrm{ft}$ & $\begin{array}{l}\text { construction } \\
\text { fill; original } \\
\text { surface } \\
\end{array}$ & $\begin{array}{l}\text { glass, brick, sewer } \\
\text { pipe }\end{array}$ \\
\hline & $\begin{array}{l}\text { demolition of Solis } \\
\text { house }\end{array}$ & Solis house & $1-2 \mathrm{ft}$ & \begin{tabular}{|l} 
construction \\
fill \\
\end{tabular} & none \\
\hline \multirow[t]{3}{*}{ 4-Dec 14} & shallow grading & southern project area & $1 \mathrm{ft}$ & $\begin{array}{l}\text { construction } \\
\text { fill }\end{array}$ & none \\
\hline & $\begin{array}{l}\text { cleaning of two } \\
\text { bioswale pits }\end{array}$ & southern project area & $3-5 \mathrm{ft}$ & $\begin{array}{l}\text { construction } \\
\text { fill; original } \\
\text { surface } \\
\end{array}$ & $\begin{array}{l}\text { D’hanis brick, yellow } \\
\text { brick }\end{array}$ \\
\hline & $\begin{array}{l}\text { excavation of Ditch } \\
\text { Witch bore pit }\end{array}$ & southern project area & $8-10 \mathrm{ft}$ & $\begin{array}{l}\text { construction } \\
\text { fill; original } \\
\text { surface }\end{array}$ & $\begin{array}{l}\text { D'hanis brick, yellow } \\
\text { brick }\end{array}$ \\
\hline 5-Dec 14 & $\begin{array}{l}\text { excavation of Ditch } \\
\text { Witch bore pit }\end{array}$ & southern project area & $8-10 \mathrm{ft}$ & $\begin{array}{l}\text { construction } \\
\text { fill; original } \\
\text { surface }\end{array}$ & $\begin{array}{l}\text { D’hanis brick, yellow } \\
\text { brick }\end{array}$ \\
\hline \multirow[t]{2}{*}{ 8-Dec 14} & $\begin{array}{l}\text { excavation of } \\
\text { manhole }\end{array}$ & southern project area & $8-10 \mathrm{ft}$ & $\begin{array}{l}\text { construction } \\
\text { fill; original } \\
\text { surface }\end{array}$ & none \\
\hline & $\begin{array}{l}\text { excavation of storm } \\
\text { sewer trench }\end{array}$ & southern project area & $12 \mathrm{ft}$ & $\begin{array}{l}\text { construction } \\
\text { fill; original } \\
\text { surface }\end{array}$ & none \\
\hline \multirow[t]{2}{*}{ 9-Dec 14} & $\begin{array}{l}\text { excavation of pit } \\
\text { along sewer line }\end{array}$ & southern project area & $13 \mathrm{ft}$ & $\begin{array}{l}\text { construction } \\
\text { fill; original } \\
\text { surface }\end{array}$ & none \\
\hline & $\begin{array}{l}\text { excavation of } \\
\text { manhole }\end{array}$ & old parking lot & $13 \mathrm{ft}$ & $\begin{array}{l}\text { construction } \\
\text { fill; original } \\
\text { surface } \\
\end{array}$ & brick fragments \\
\hline \multirow[t]{3}{*}{ 10-Dec 14} & $\begin{array}{l}\text { excavation of } \\
\text { manhole }\end{array}$ & old parking lot & $13 \mathrm{ft}$ & $\begin{array}{l}\text { construction } \\
\text { fill; original } \\
\text { surface }\end{array}$ & none \\
\hline & $\begin{array}{l}\text { excavation of welding } \\
\text { pit for line casing }\end{array}$ & east of acequia & $13 \mathrm{ft}$ & $\begin{array}{l}\text { construction } \\
\text { fill; original } \\
\text { surface } \\
\end{array}$ & none \\
\hline & $\begin{array}{l}\text { excavation of } \\
\text { bioswale pit } \\
\end{array}$ & $\begin{array}{l}\text { north end of project } \\
\text { area }\end{array}$ & $5 \mathrm{ft}$ & $\begin{array}{l}\text { numerous } \\
\text { layers of fill }\end{array}$ & red bricks \\
\hline \multirow[t]{2}{*}{ 11-Dec 14} & $\begin{array}{l}\text { clean up of bioswale } \\
\text { pit }\end{array}$ & $\begin{array}{l}\text { north end of project } \\
\text { area }\end{array}$ & $5 \mathrm{ft}$ & $\begin{array}{l}\text { numerous } \\
\text { layers of fill }\end{array}$ & red bricks \\
\hline & $\begin{array}{l}\text { excavation/clean up of } \\
\text { casing pits }\end{array}$ & east of acequia & $13 \mathrm{ft}$ & $\begin{array}{l}\text { construction } \\
\text { fill; original } \\
\text { surface } \\
\end{array}$ & none \\
\hline 12-Dec 14 & $\begin{array}{l}\text { excavation of Ditch } \\
\text { Witch bore pit }\end{array}$ & southern project area & $8 \mathrm{ft}$ & $\begin{array}{l}\text { construction } \\
\text { fill; original } \\
\text { surface } \\
\end{array}$ & none \\
\hline
\end{tabular}


Table 2, continued

\begin{tabular}{|c|c|c|c|c|c|}
\hline Date & Activities Monitored & $\begin{array}{l}\text { Location of } \\
\text { Monitoring }\end{array}$ & $\begin{array}{l}\text { Depth of } \\
\text { Impacts }\end{array}$ & $\begin{array}{c}\text { Nature of } \\
\text { Deposits }\end{array}$ & $\begin{array}{c}\text { Archeological } \\
\text { Remains } \\
\end{array}$ \\
\hline \multirow[t]{2}{*}{ 15-Dec 14} & $\begin{array}{l}\text { trenching for } \\
\text { irrigation sleeves }\end{array}$ & $\begin{array}{l}\text { throughout project } \\
\text { area }\end{array}$ & $1.5 \mathrm{ft}$ & $\begin{array}{l}\text { construction } \\
\text { fill } \\
\end{array}$ & none \\
\hline & excavation of bore pit & southern project area & $8 \mathrm{ft}$ & $\begin{array}{l}\text { construction } \\
\text { fill; original } \\
\text { surface }\end{array}$ & none \\
\hline \multirow[t]{4}{*}{ 16-Dec 14} & $\begin{array}{l}\text { excavation of } \\
\text { sewer line bore pit } \\
\text { extension }\end{array}$ & southern project area & $8 \mathrm{ft}$ & $\begin{array}{l}\text { construction } \\
\text { fill; original } \\
\text { surface } \\
\end{array}$ & none \\
\hline & removal of curb & near Carriage house & $1.5 \mathrm{ft}$ & $\begin{array}{l}\text { construction } \\
\text { fill } \\
\end{array}$ & none \\
\hline & $\begin{array}{l}\text { excavation of Feature } \\
1 \text { at } 41 \mathrm{BX} 2068\end{array}$ & southern project area & $2-4 \mathrm{ft}$ & cultural fill & $\begin{array}{l}\text { various types of } \\
\text { household refuse }\end{array}$ \\
\hline & $\begin{array}{l}\text { excavation around } \\
\text { oak tree to be } \\
\text { transplanted } \\
\end{array}$ & police parking lot & $5 \mathrm{ft}$ & $\begin{array}{l}\text { construction } \\
\text { fill }\end{array}$ & none \\
\hline \multirow[t]{3}{*}{ 17-Dec 14} & $\begin{array}{l}\text { excavation of small } \\
\text { trench for water line }\end{array}$ & southern project area & $1-4 \mathrm{ft}$ & $\begin{array}{l}\text { construction } \\
\text { fill; original } \\
\text { surface }\end{array}$ & none \\
\hline & $\begin{array}{l}\text { excavation of Feature } \\
1 \text { at } 41 \mathrm{BX} 2068\end{array}$ & southern project area & $2-4 \mathrm{ft}$ & cultural fill & $\begin{array}{l}\text { various types of } \\
\text { household refuse }\end{array}$ \\
\hline & $\begin{array}{l}\text { demolition of } \\
\text { manhole }\end{array}$ & north end of acequia & $3 \mathrm{ft}$ & all concrete & none \\
\hline \multirow[t]{2}{*}{ 18-Dec 14} & $\begin{array}{l}\text { excavation of sewer } \\
\text { trench }\end{array}$ & old parking lot & $13 \mathrm{ft}$ & $\begin{array}{l}\text { construction } \\
\text { fill; original } \\
\text { surface }\end{array}$ & none \\
\hline & $\begin{array}{l}\text { removal of brick } \\
\text { pavers }\end{array}$ & $\begin{array}{l}\text { between Carriage } \\
\text { house and Eager } \\
\text { Dependency }\end{array}$ & $1 \mathrm{ft}$ & $\begin{array}{l}\text { construction } \\
\text { fill }\end{array}$ & none \\
\hline 19-Dec 14 & \begin{tabular}{|l} 
no ground \\
disturbance
\end{tabular} & N/A & N/A & N/A & N/A \\
\hline 22-Dec 14 & $\begin{array}{l}\text { extension of sewer } \\
\text { trench }\end{array}$ & old parking lot & $13 \mathrm{ft}$ & $\begin{array}{l}\text { construction } \\
\text { fill; original } \\
\text { surface }\end{array}$ & none \\
\hline 23-Dec 14 & $\begin{array}{l}\text { no ground } \\
\text { disturbance }\end{array}$ & N/A & N/A & N/A & N/A \\
\hline 24-Dec 14 & $\begin{array}{l}\text { removal of brick } \\
\text { pavers and curb }\end{array}$ & $\begin{array}{l}\text { north and east of } \\
\text { Eager house }\end{array}$ & $1.5 \mathrm{ft}$ & $\begin{array}{l}\text { construction } \\
\text { fill }\end{array}$ & none \\
\hline
\end{tabular}

excavator (Figure 10b). The footings left behind were removed as well, resulting in 1-2 ft of fill being disturbed.

Brick Paver Removal: The many walkways throughout the park were built of brick pavers. Pavers were laid in an interlocking pattern between concrete curbs. Each brick measured 3 inches thick and sat upon a layer of leveling sand. These impacts were very shallow, generally no deeper than $0.5 \mathrm{ft}$. Bricks were removed using a small excavator and Bobcat.

Concrete Curb Removal: Hundreds of linear feet of concrete curbs snaked throughout the park (see Figure 10b). Curbs typically reached ca. $1.5 \mathrm{ft}$ deep and were removed using the excavator. A narrow bucket with teeth was used to pull out cut segments of curbs. 
Shrub Removal: Many small shrubs throughout the park had to be removed, and this was accomplished using an excavator and backhoe. Many of the root systems did not reach beyond $1 \mathrm{ft}$ deep and were easily pulled out with the machine.

Pavement Removal: Using Bobcats, a backhoe, and an excavator, the parking lot between the Eager and Pereida houses was peeled away (Figure 11a). The pavement measured ca. 3-4 inches thick and sat on top of a layer of caliche base.

Restroom Building and Foundation Demolition:The former women's restroom within the park was demolished using a large excavator. There were no subsurface impacts as the building was destroyed, but the 2 -ft-thick concrete foundation had to be broken up with a jackhammer and removed by machine.

Site Grading: Site grading was accomplished by using a team of Bobcats to cut away the upper few inches of introduced fill to bring the ground surface to its new elevation (Figure 11b; Figure 12). Impacts from grading were typically less than $1 \mathrm{ft}$.

Irrigation Sleeve Trenching: Irrigation sleeves (PVC pipes 4-6 inches in diameter) were laid in the ground to encase irrigation lines where they will pass under roadways and sidewalks. These trenches were excavated using a Ditch Witch trencher and went to depths of ca. $1.5 \mathrm{ft}$.

\section{Monitoring of Deep Impacts}

Deep subsurface impacts were less pervasive but often occurred on a larger scale and removed hundreds of cubic yards of material. The primary deep impacts are listed below, and the locations of the more-prominent ones are shown on Figure 9.

Tree Removal: Twenty-three trees were selected for removal within Yanaguana Garden. They ranged in size from small crepe myrtles ca. 5 inches in diameter up to a large pecan tree 34 inches in diameter. These trees were removed by an excavator, which toppled the trees once the large branches were removed. In most instances, impacts from removing a tree's root ball reached between 3 and $6 \mathrm{ft}$ deep and were 3-8 $\mathrm{ft}$ in diameter. In all cases, removing the root ball disturbed the ground to the extent that no informative subsurface profiles could be observed or recorded.

Light Pole Base Removal: Large light poles scattered throughout the park were removed. The cast-iron poles were easily dismantled, leaving behind 1.5 -ftdiameter concrete bases that reached $3-5 \mathrm{ft}$ below the surface. These concrete columns were removed using an excavator, sometimes leaving a perfectly circular hole, except where additional excavation was needed to extract the base. In general, the resulting holes did not provide informative subsurface profiles because they were too small or too disturbed by machinery in the excavation process.

Utility Potholing: Potholing was conducted to try and find some of the many unmarked buried utility lines throughout the project area. A vacuum truck and attached high-pressure blower hose were used to excavate and remove sediments to depths of 4-12 ft in a few areas. The holes were too small in diameter to allow observing and recording subsurface profiles. On occasion when unmarked utilities 


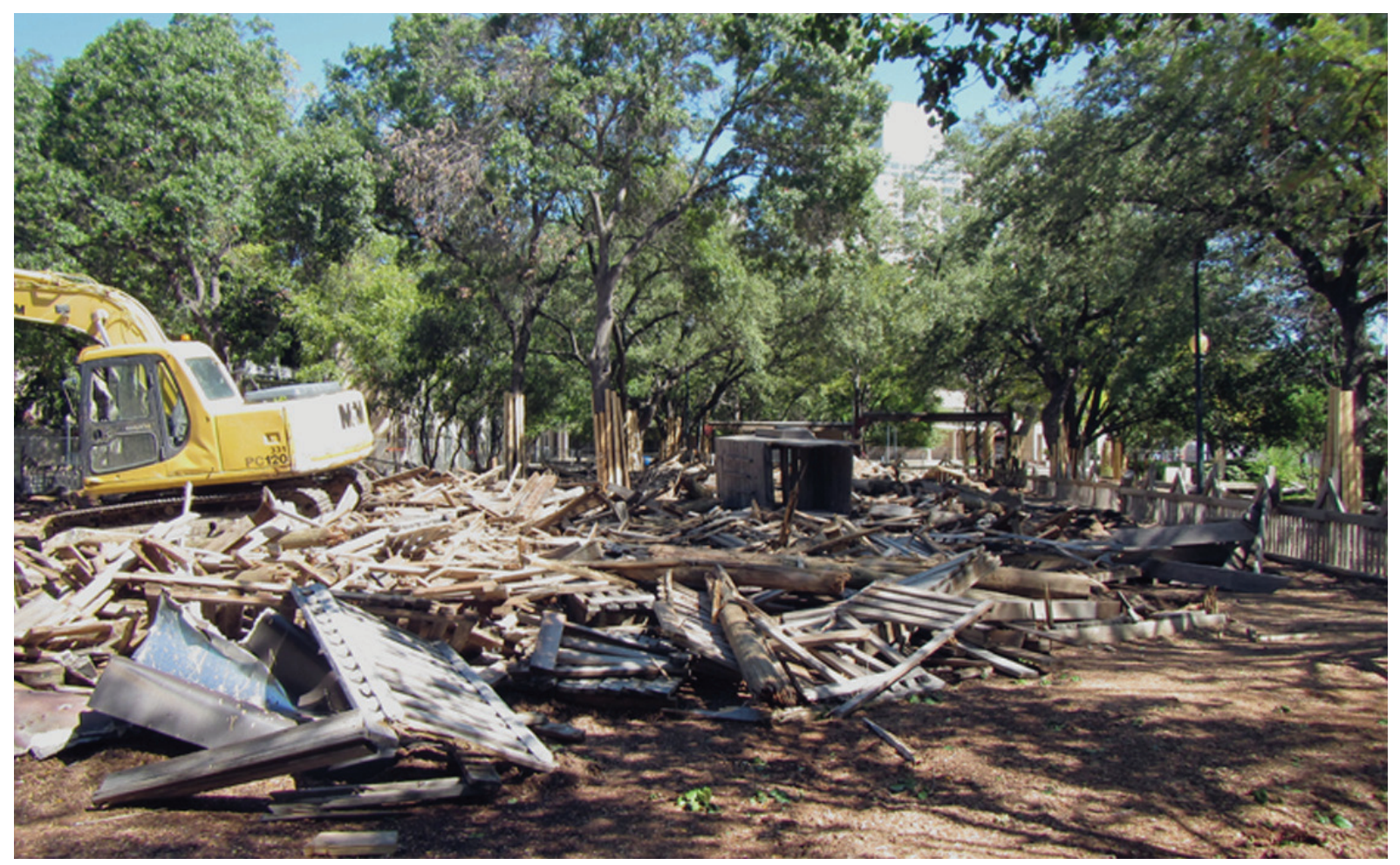

a

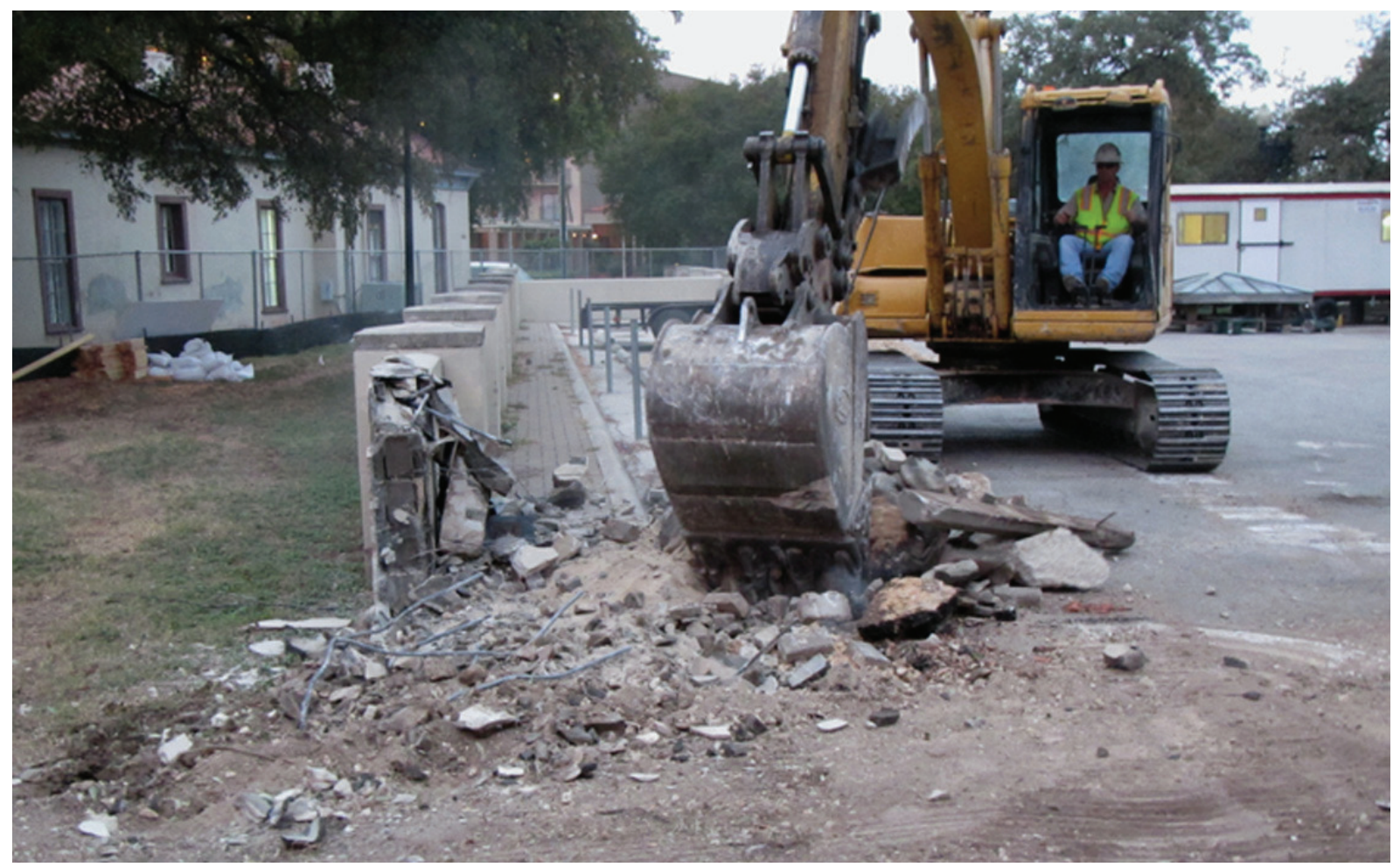

b

Figure 10. Examples of construction activities that caused shallow subsurface impacts. (a) View to the northeast of demolition of the playscape in the central part of the project area; (b) view to the west of an excavator removing a masonry wall, brick paver sidewalk, and concrete curb at the south edge of the parking lot between the Eager and Pereida houses. 


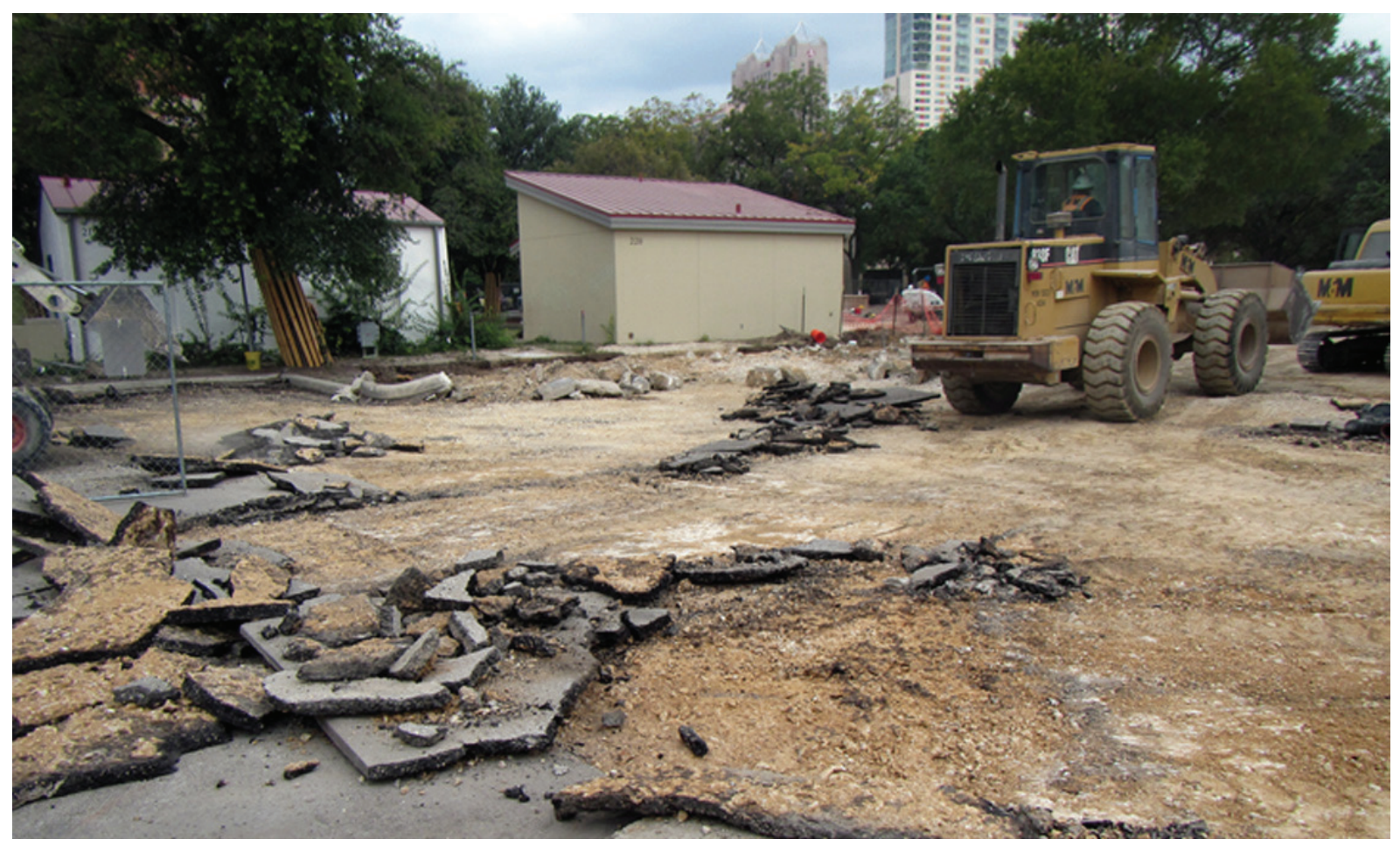

a

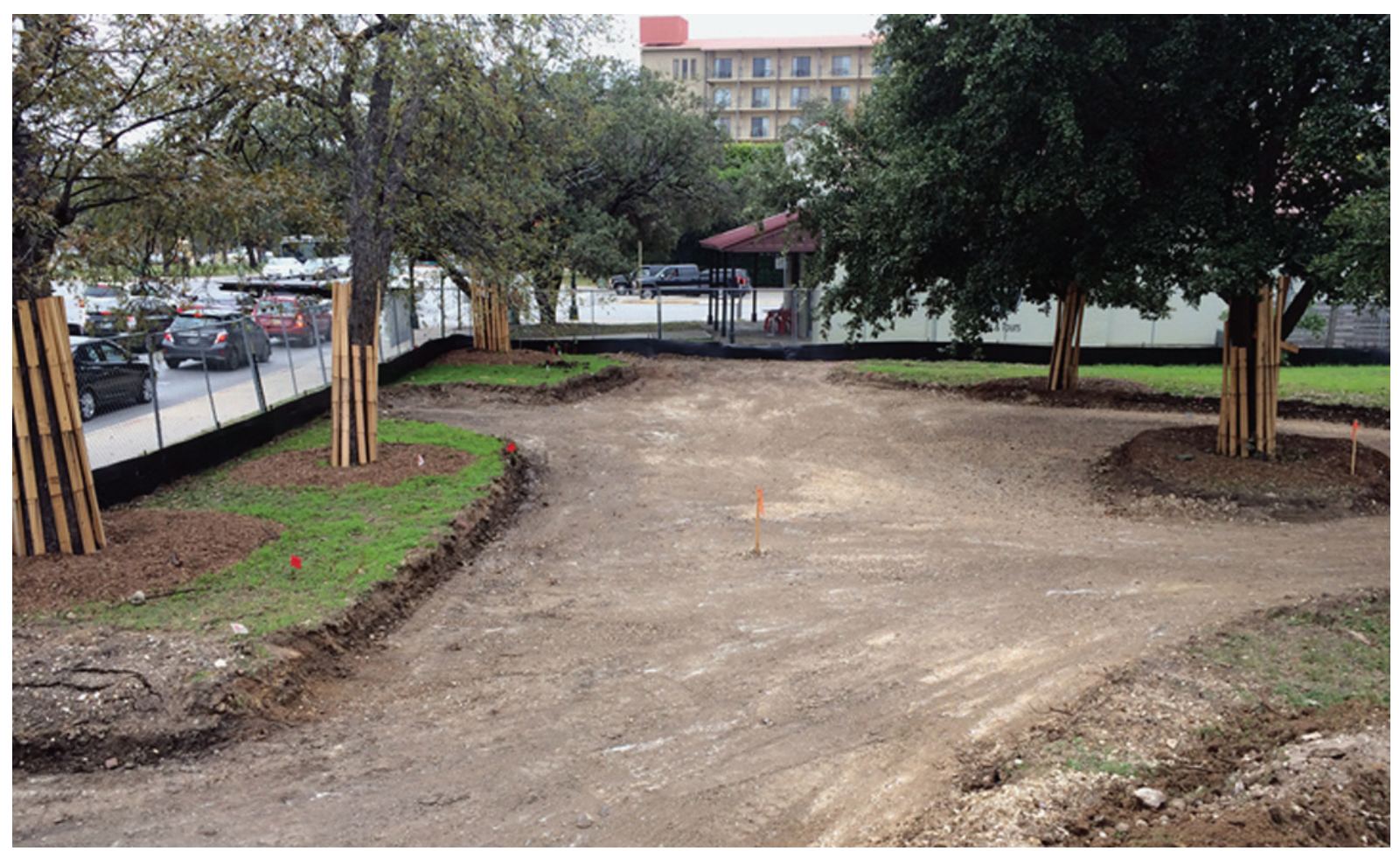

$\mathrm{b}$

Figure 11. Examples of construction activities that caused shallow subsurface impacts. (a) View to the northeast of a loader and excavator removing the pavement from the parking lot between the Eager and Pereida houses (note caliche base just beneath pavement); (b) view to the west of shallow grading for future walkways at the south edge of Yanaguana Garden. 


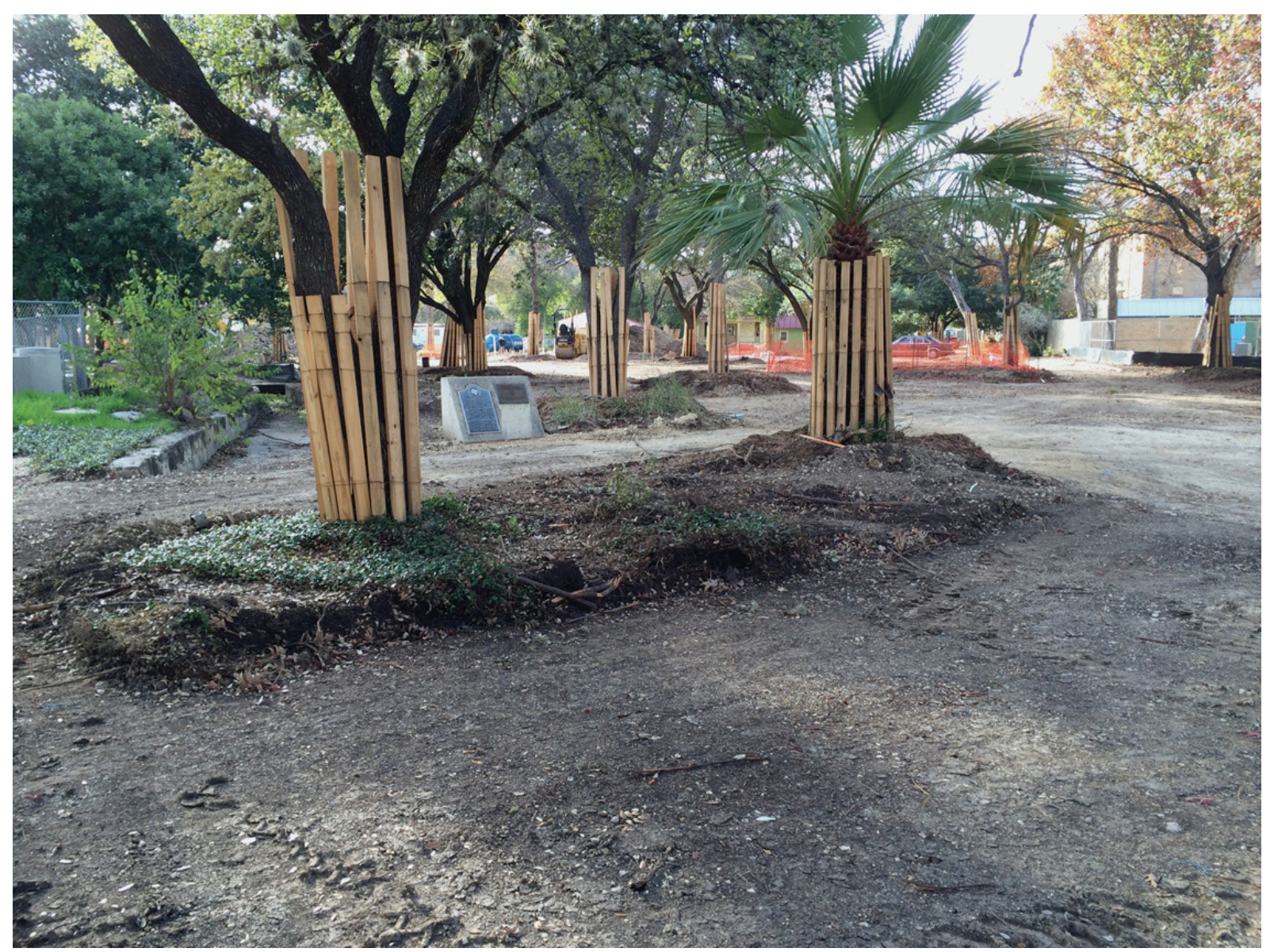

Figure 12. View to the southwest of shallow impacts caused by grading for walkways at the north end of the project area. The north end of the reconstructed Acequia Madre is just beyond the nearest tree, and the historical marker for the acequia is to the right of it.

were identified, workers dug by hand or with a Bobcat to expose additional segments of the utility line. These utilities were close to the surface, often in introduced fill.

New Restroom Pad Excavation: A new restroom was to be built directly over the site of the old, demolished restroom, thus limiting impacts to an area that had already been disturbed. A large pit measuring $45 \times 45 \mathrm{ft}$ and $4 \mathrm{ft}$ deep was excavated so that it could be packed with caliche base and serve as the pad for the new foundation (Figure 13). This pit was dug by an excavator and a team of Bobcats. One unexpected feature encountered was a large concrete pier measuring at least 10-12 ft tall and 1-2 ft in diameter with a bell-shaped base measuring 2-7 ft across (Figure 14). At least five other such large piers were unearthed during monitoring: one just southeast of the new restroom, one at the manhole pit within the former parking lot, and three during bore pit and sewer line excavation in the southern portion of the project area. It is unclear what these massive concrete columns were associated with, but they probably related to HemisFair '68 construction; four of them were beneath or adjacent to temporary HemisFair '68 buildings. 


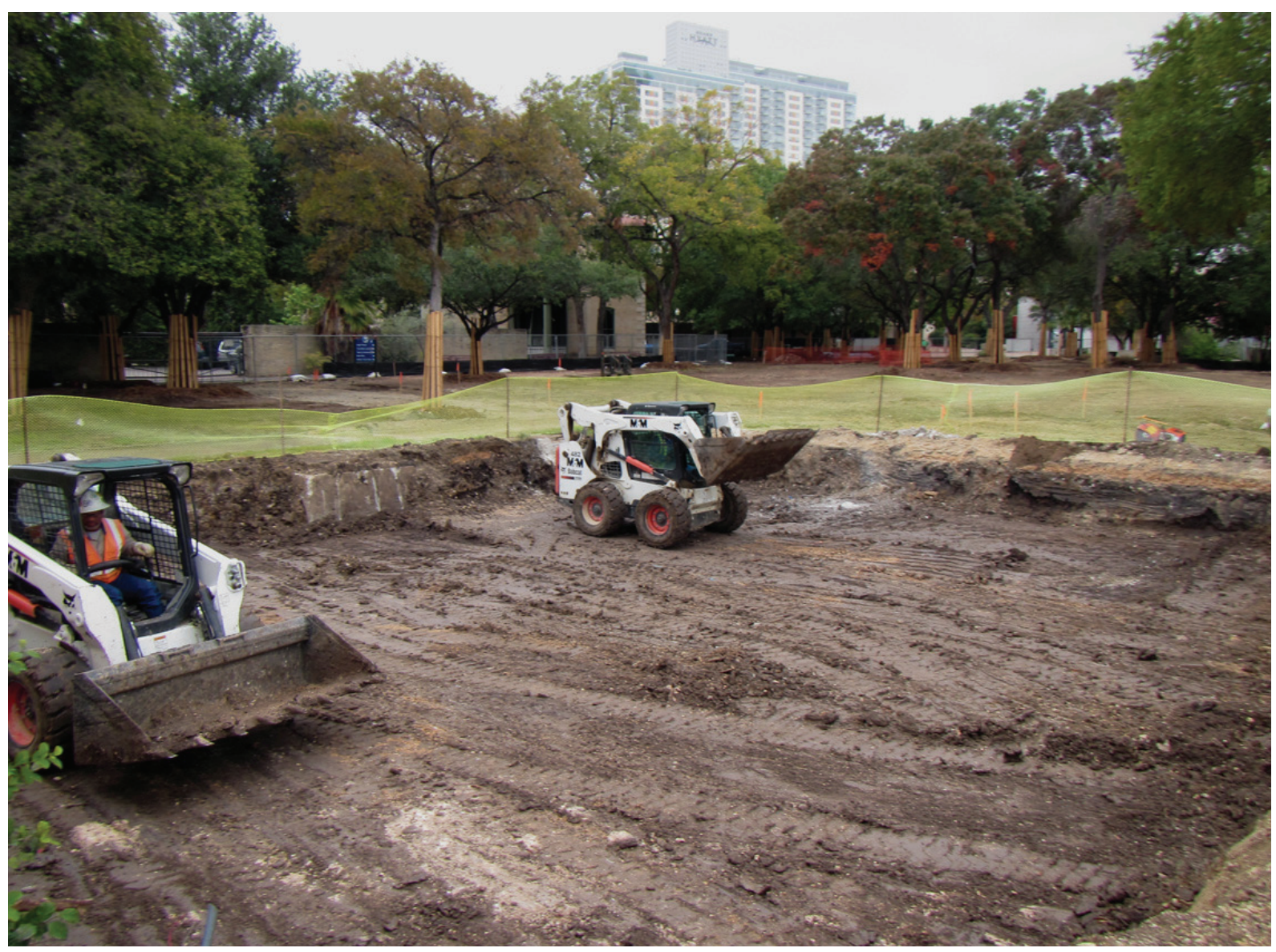

Figure 13. View to the northeast of Bobcats excavating 4-ft-deep new restroom pad.

Bioswale Pit Excavation: Three large bioswale pits were excavated, one in the north part of the project area and two nearby in the south part (Figure 15a). The north pit measured ca. $25 \mathrm{ft}$ long by $10 \mathrm{ft}$ wide, and the two south pits were similar in size measuring ca. $25 \mathrm{ft}$ long by $8-10 \mathrm{ft}$ wide. Each pit was excavated to a depth of $4-5 \mathrm{ft}$ below the surface. A large excavator and team of Bobcats were used to accomplish the task.

Sanitary Sewer Trench Excavation: The deepest and most extensive impacts monitored occurred in connection with trenching for new sanitary sewer lines. Approximately 575 linear feet of new sanitary sewer lines were planned for the project. At the completion of our monitoring, about 25 percent of these trenches had been dug. Trenches were excavated using a large excavator to set the pipes. Sewer line trenches measured ca. $4 \mathrm{ft}$ wide and 12-14 ft deep. One segment monitored was partially within the old parking lot and south and east of the new restroom building, while other segments were in the southern portion of the project area. Accompanying the sewer line trenching was excavation of two manhole pits using a large excavator. These manholes serve as access points along the sanitary sewer system. Each pit measured ca. $8 \times 8 \mathrm{ft}$ and reached depths of 12-13 ft. 
Two segments of planned sanitary sewer lines in the southern portion of the project area intersected the alignment of the Acequia Madre. Although trenching had determined that most of the acequia in this area had been disturbed or destroyed, CoSA decided that the lines should be bored beneath the alignment to ensure that no remaining traces of the acequia would be impacted. This necessitated excavating two large pits (12x25 ft and $8 \mathrm{ft}$ deep) with a large excavator to accommodate a Ditch Witch machine (Figure 15b). This machine then bored two 30-inch-diameter holes so that 24-inch iron casings could be placed to house the 8-inch sewer pipes (Figure 16; only one of these casings was installed during our monitoring). An 8x8-ft welding pit was dug in one location so that the bored head could be retrieved and to allow the casing to be welded in place.

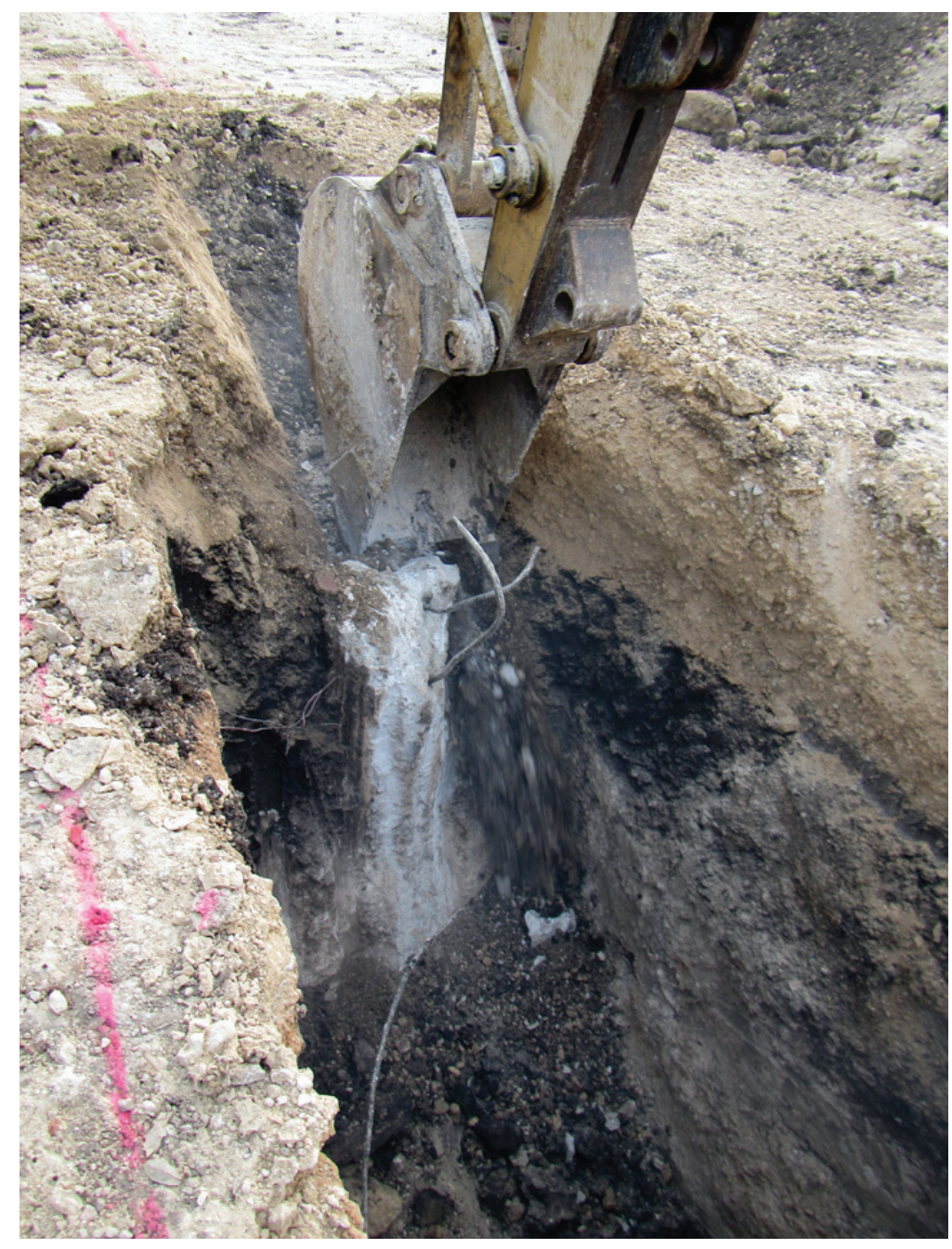

Figure 14. Photograph of excavator removing the upper part of one of six deep (10-12 ft) concrete piers. The pier extended beneath introduced fill into in situ dark sediments and Austin Chalk bedrock beneath. 


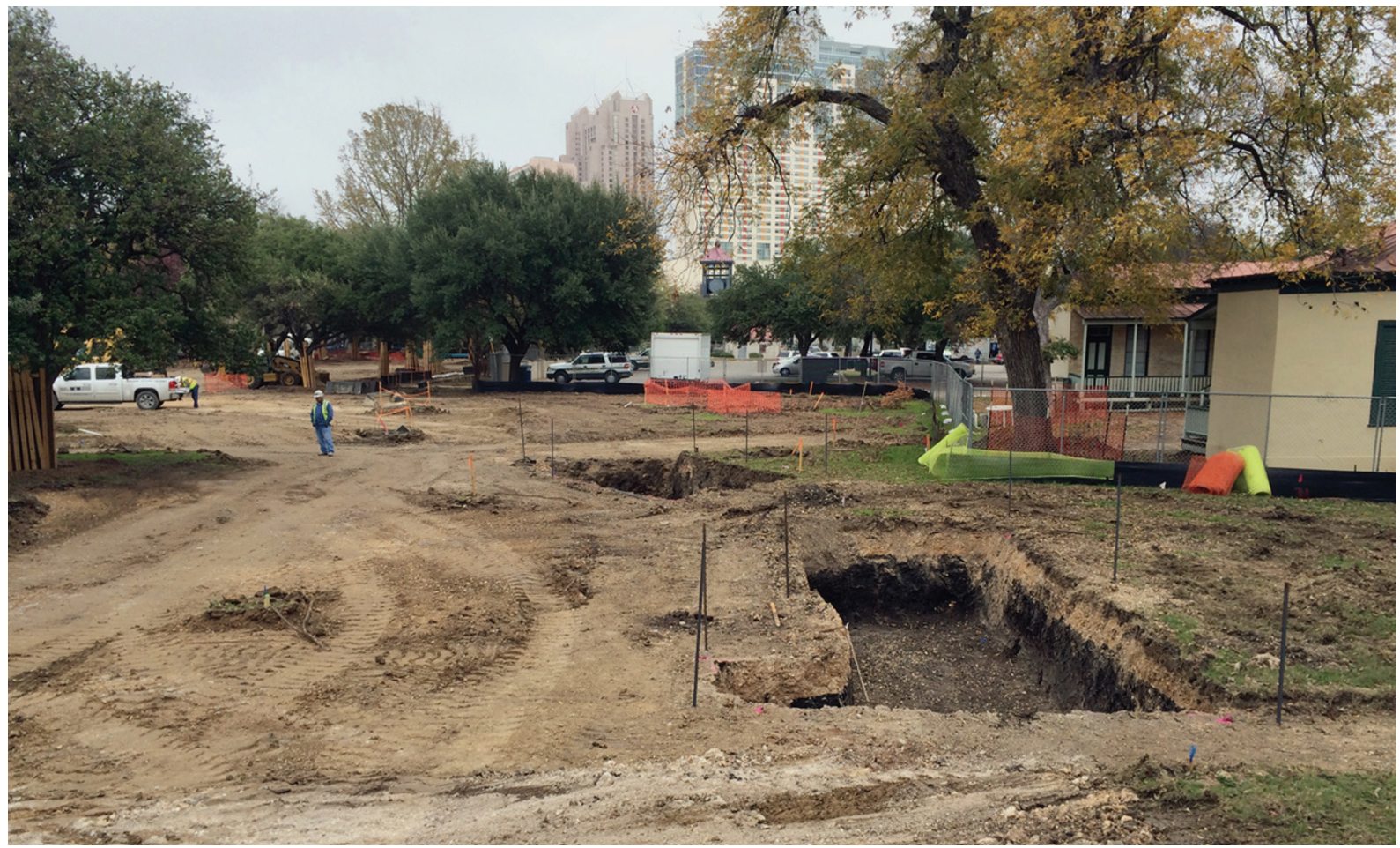

a

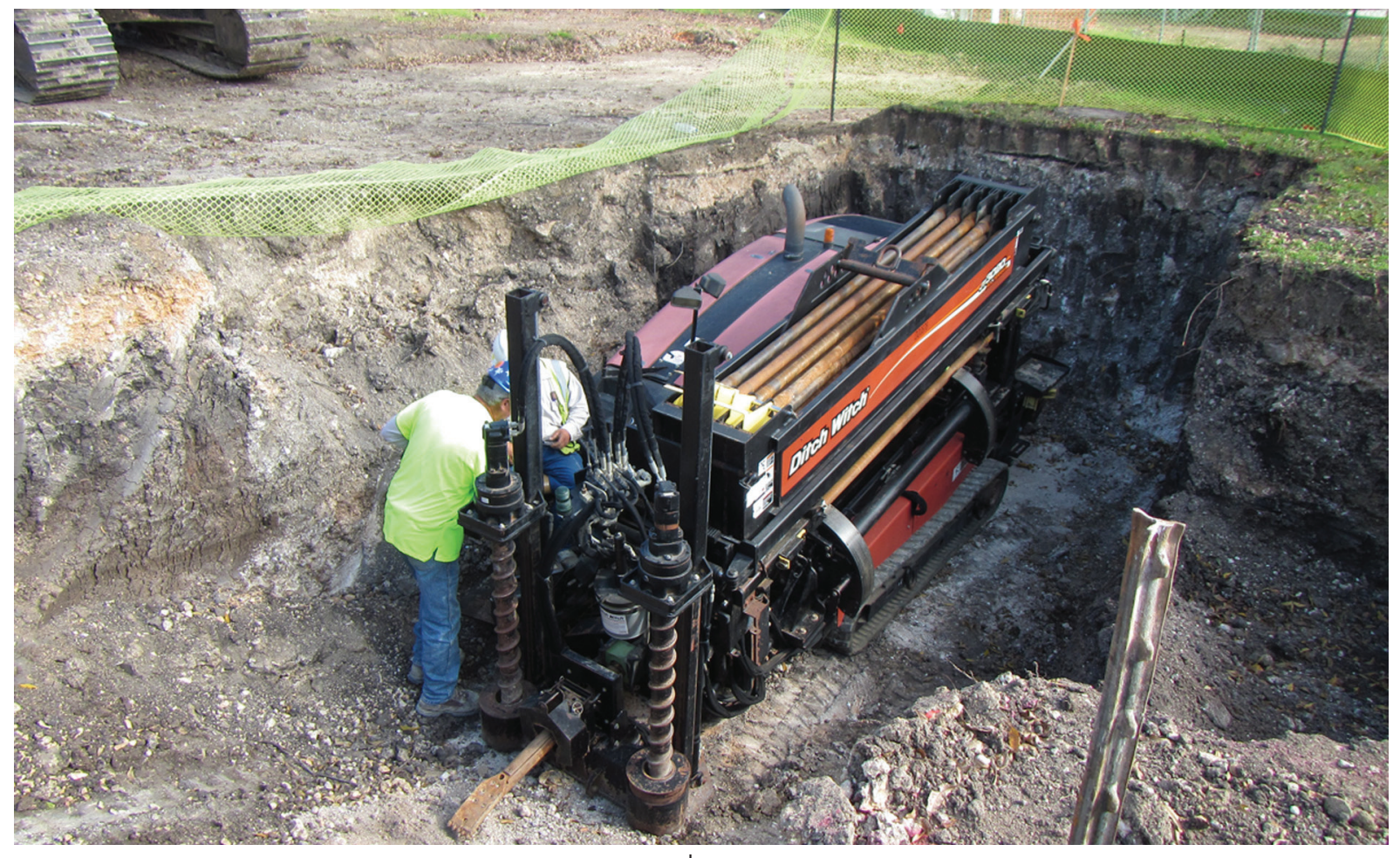

b

Figure 15. Examples of construction activities that caused deep subsurface impacts. (a) View to the northeast of two 5-ft-deep southern bioswale excavations behind the Koehler house (note shallow impacts from site grading nearby); (b) view to the east of 8-ft-deep bore pit excavation for Ditch Witch machine behind the Koehler house (this pit transected one of the southern bioswale pits, visible to the right). 


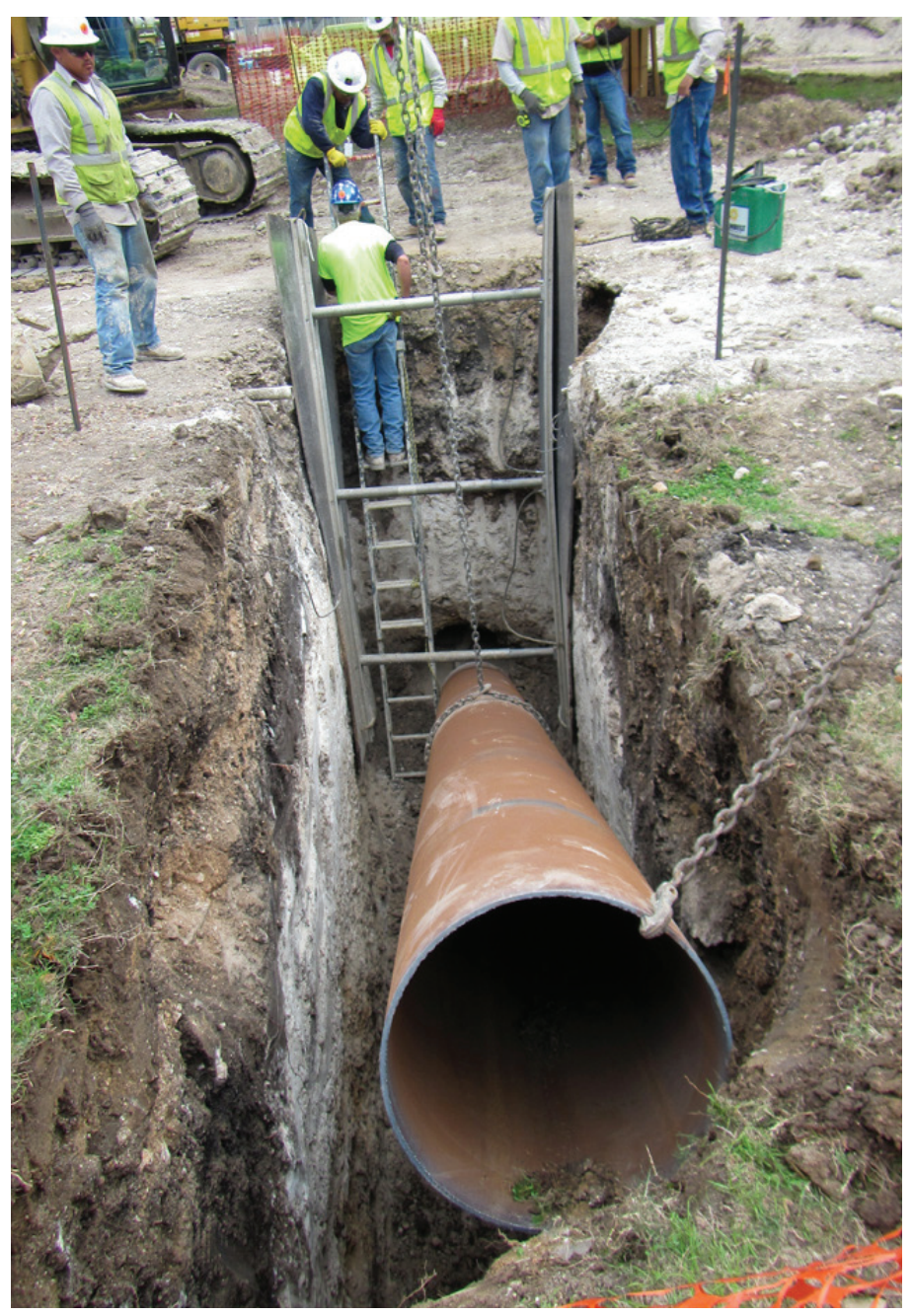

Figure 16. View of deep excavation required for placement of casing to hold sewer line that was bored beneath the acequia alignment in the south part of the project area. 


\section{CHAPTER 4: RESULTS OF ARCHEOLOGICAL INVESTIGATIONS}

Introduced fill is ubiquitous across the project area, and much of the monitoring was for activities that impacted only this fill. Modern construction debris and rubble, such as brick and concrete fragments and rebar, and modern trash in the form of plastic cups, bags, and glass bottles were observed frequently in this fill. On occasion, nonmodern artifacts were observed, but only where impacts reached beneath the fill layers. These consisted of items like glass bottle fragments, ceramic sherds, nails, animal bones, ceramic sewer pipe fragments, bricks, and rusted metal. Except for a few rare instances (see 41BX8 and 41BX2068 below), these artifacts were in disturbed contexts and likely represent a general trash scatter relating to latenineteenth- and early-to-mid-twentieth-century occupation of the area; undoubtedly, these materials had been displaced by the multiple episodes of earthmoving before and after HemisFair '68. The only substantive archeological results came from trenching in search of the Acequia Madre (41BX8) and excavation of a nineteenthcentury trash pit (41BX2068) identified during monitoring.

\section{OVERALL STRATIGRAPHY}

The deeper subsurface profiles traced the history of modification of this corner of HemisFair Park. Table 3 summarizes the more-informative of these profiles, including their locations, the stratigraphy observed (including depth of disturbance), and archeological remains observed. Figure 9 shows the locations of these profiles.

As the background research revealed, the area has seen extensive, and in some areas quite deep, disturbance. All 15 of the profiles listed in Table 3 contain introduced fill in their upper parts, ranging in thickness from 1.0 to $5.9 \mathrm{ft}$ and averaging $2-4 \mathrm{ft}$. This fill varies from sediment and mulch used in landscaping, to caliche and gravel base material, to construction debris (concrete, rebar, etc.) mixed with dark clayey sediments (Figures 17 and 18). It contains many buried utility lines. Common modern artifacts and occasional historic artifacts were observed in this fill, with the latter always being out of context.

Below the fill in almost all of the profiles are natural clayey sediments classified as Houston Black clay, which are upland soils that are dark gray to black and calcareous, often reaching 3-4 ft deep (Taylor et al. 1991:18-19). In many profiles, these sediments have been disturbed by numerous buried utilities and the addition of construction debris. This undoubtedly reflects the many episodes of earthmoving and landscape modification associated mostly with HemisFair '68 construction and demolition. In some cases, disturbance is limited to the upper part of the profile, 
Table 3. Summary of deeper sediment profiles at Yanaguana Garden

\begin{tabular}{|c|c|c|}
\hline Location & Profile Description & Archeological Remains \\
\hline $\begin{array}{l}\text { North Emergency } \\
\text { Service Drive }\end{array}$ & $\begin{array}{l}\text { introduced fill to } 2.0-4.6 \mathrm{ft} \text {; in situ Houston Black clay } \\
\text { below the fill, with Austin Chalk bedrock at } 4.3 \mathrm{ft}\end{array}$ & $\begin{array}{l}\text { transferware, glass bottle neck, horseshoe, } \\
\text { yellow bricks (all out of context) }\end{array}$ \\
\hline $\begin{array}{l}\text { Removal of } \\
\text { Contaminated Soils- } \\
\text { Area } 1\end{array}$ & $\begin{array}{l}\text { asphalt and gravel fill to } 1.0-1.5 \mathrm{ft} \text {; disturbed } \\
\text { Houston Black clay with construction debris to } 2.5 \mathrm{ft}\end{array}$ & glass ink bottle \\
\hline $\begin{array}{l}\text { Removal of } \\
\text { Contaminated Soils- } \\
\text { Area } 2\end{array}$ & $\begin{array}{l}\text { introduced fill to } 2.0-2.8 \mathrm{ft} \text {; disturbed Houston Black } \\
\text { clay to } 3.0-4.0 \mathrm{ft} \text {; in situ Houston Black clay at } 4.0- \\
4.6 \mathrm{ft} \text {; Austin Chalk bedrock at } 4.6 \mathrm{ft} \\
\end{array}$ & $\begin{array}{l}\text { shaped limestone, animal bones, metal, } \\
\text { glass bottle fragments }\end{array}$ \\
\hline $\begin{array}{l}\text { Acequia Trenching- } \\
\text { Trench PAI-1 }\end{array}$ & $\begin{array}{l}\text { introduced fill to } 2.3-4.3 \mathrm{ft} \text {;in situ Houston Black clay } \\
\text { below } 2.3 \mathrm{ft} \text { east of acequia; dark clayey acequia ditch } \\
\text { fill at } 4.3-5.4 \mathrm{ft} \text {; Austin Chalk bedrock at } 5.4 \mathrm{ft}\end{array}$ & $\begin{array}{l}\text { rock wall of Acequia Madre starting at } \\
3.0-3.3 \mathrm{ft} \text { below the surface; metal, glass } \\
\text { bottle fragments, ceramics in acequia } \\
\text { ditch fill }\end{array}$ \\
\hline $\begin{array}{l}\text { Acequia Trenching- } \\
\text { Trench PAI-2 }\end{array}$ & $\begin{array}{l}\text { disturbed fill to } 5.2 \mathrm{ft} \text {; Austin Chalk bedrock below } \\
5.2 \mathrm{ft}\end{array}$ & none \\
\hline $\begin{array}{l}\text { Acequia Trenching- } \\
\text { Trench PAI-3 }\end{array}$ & $\begin{array}{l}\text { introduced fill to } 4.6 \mathrm{ft} \text {; probable acequia ditch fill at } \\
4.6-6.6 \mathrm{ft} \text {, inset into in situ Houston Black clay on } \\
\text { the east side at } 4.6 \mathrm{ft} \text { and caliche bedrock on the west } \\
\text { side at } 5.2 \mathrm{ft}\end{array}$ & $\begin{array}{l}\text { metal and glass in probable acequia ditch } \\
\text { fill }\end{array}$ \\
\hline $\begin{array}{l}\text { Acequia Trenching- } \\
\text { Trench PAI-4 }\end{array}$ & $\begin{array}{l}\text { introduced fill to } 3.3 \mathrm{ft} \text {; dark clayey acequia ditch } \\
\text { fill with construction rubble to } 5.2 \mathrm{ft} \text {; Austin Chalk } \\
\text { bedrock below } 5.2 \mathrm{ft}\end{array}$ & $\begin{array}{l}\text { rock wall of Acequia Madre starting at } \\
3.0-3.3 \mathrm{ft} \text { below the surface; glass bottle } \\
\text { fragments, ceramics, metal, animal bones, } \\
\text { bricks in acequia ditch fill }\end{array}$ \\
\hline $\begin{array}{l}\text { Acequia Trenching- } \\
\text { Trench PAI-5 }\end{array}$ & $5.9 \mathrm{ft}$ of fill and/or heavily disturbed soils & none \\
\hline $\begin{array}{l}\text { Acequia Trenching- } \\
\text { Trench PAI-6 }\end{array}$ & $5.9 \mathrm{ft}$ of fill and/or heavily disturbed soils & $\begin{array}{l}\text { glass bottle fragments, ceramic, brick, axe } \\
\text { head }\end{array}$ \\
\hline Bioswale Pit 1 & $\begin{array}{l}\text { introduced fill to } 3.6 \mathrm{ft} \text { (at least nine fill episodes); in } \\
\text { situ Houston Black clay at } 3.6 \mathrm{ft}\end{array}$ & bricks \\
\hline Restroom Pad & $\begin{array}{l}\text { introduced fill (two layers) to } 3.3 \mathrm{ft} \text {; in situ Houston } \\
\text { Black clay at ca. } 3-4 \mathrm{ft}\end{array}$ & none \\
\hline $\begin{array}{l}\text { Manhole Pit within } \\
\text { Parking Lot }\end{array}$ & $\begin{array}{l}\text { introduced fill to ca. } 2-3 \mathrm{ft} \text {; in situ Houston Black } \\
\text { clay to } 5 \mathrm{ft} \text {; Austin Chalk bedrock below } 5 \mathrm{ft}\end{array}$ & none \\
\hline $\begin{array}{l}\text { Segment of Welding/ } \\
\text { Casing Pit for Sewer } \\
\text { Line Boring }\end{array}$ & $\begin{array}{l}\text { introduced fill to } 2-4 \mathrm{ft} \text {; in situ Houston Black clay to } \\
4-6 \mathrm{ft} \text {; Austin Chalk bedrock below } 4-6 \mathrm{ft}\end{array}$ & bricks \\
\hline Bioswale Pit 2 & $\begin{array}{l}\text { introduced fill to } 1.0-1.5 \mathrm{ft} \text {; in situ Houston Black to } \\
\text { ca. } 4.5 \mathrm{ft} \text {; Austin Chalk bedrock below ca. } 4.5 \mathrm{ft}\end{array}$ & bricks \\
\hline $\begin{array}{l}\text { Bioswale Pit } 3 \text { and } \\
\text { Ditch Witch Pit }\end{array}$ & $\begin{array}{l}\text { introduced fill to } 1.0-1.5 \mathrm{ft} \text {; disturbed Houston Black } \\
\text { to ca. } 6-7 \mathrm{ft} \text {; Austin Chalk bedrock below ca. } 7 \mathrm{ft}\end{array}$ & bricks \\
\hline
\end{tabular}

but in others none of the remaining sediments are in situ. In some profiles, natural Austin Chalk deposits, the parent material in which Houston Black clay forms, were exposed at the bottom of the excavations. The only intact archeological remains found during the project were intrusive into Houston Black clay or Austin Chalk where they had not been disturbed by modern construction.

One important thing that Table 3 and the discussion above do not capture is the fact that there are areas immediately adjacent to the project, namely the environs of the various historic buildings that face onto South Alamo Street and the former courses of Goliad and Water Streets, that have escaped extensive disturbance. Although some minor leveling and filling may have been done when these houses were built, the lands next to them are at grade with the natural late-nineteenthcentury ground surface (based on their positions relative to the buildings). Given 


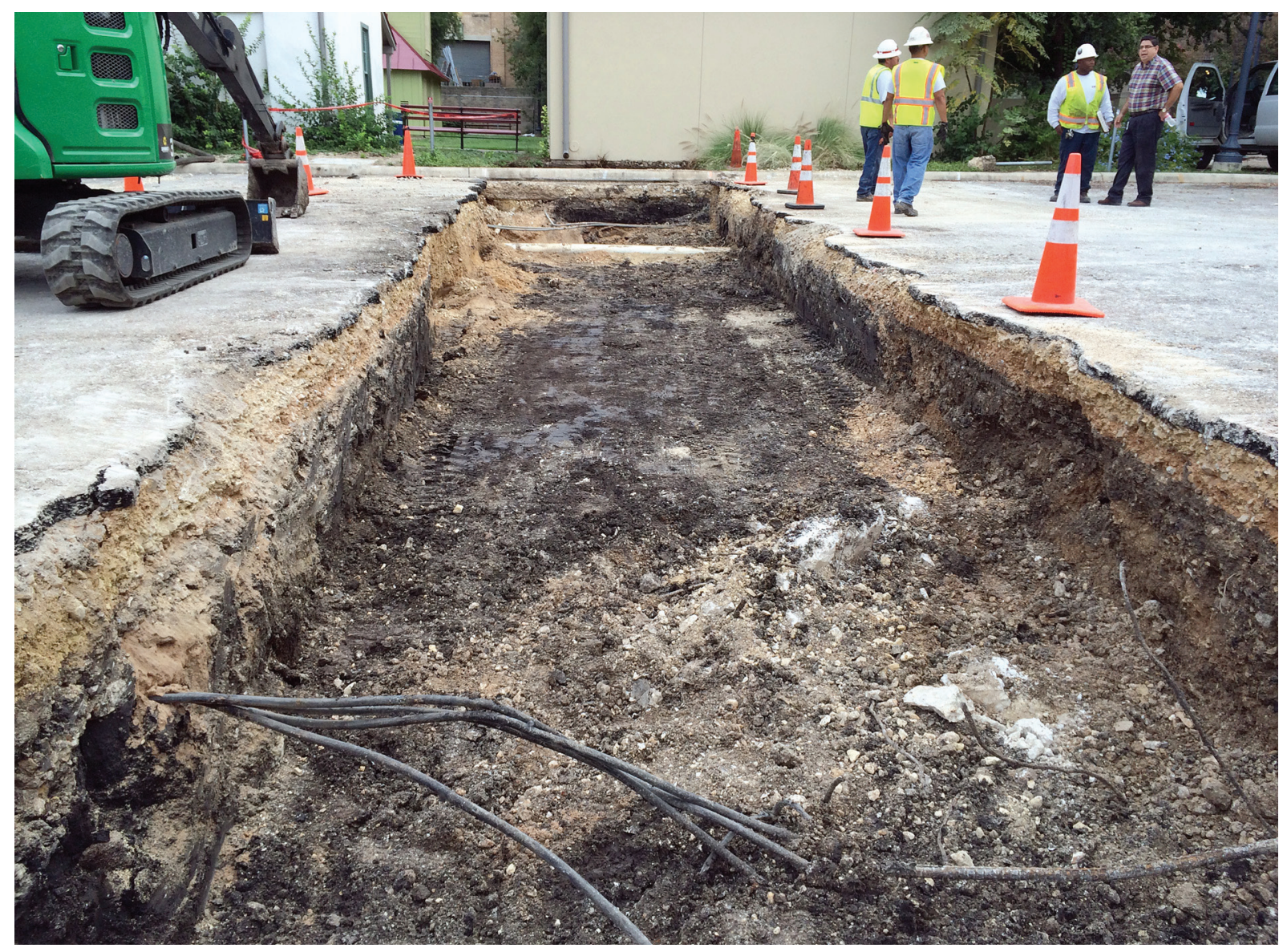

Figure 17. View to the north of the trench dug to remove contaminated soils beneath the parking lot between the Eager and Pereida houses showing subsurface stratigraphy. Parking lot pavement rests on introduced gravel base material (1.0-1.5 ft thick). Below that to $2.5 \mathrm{ft}$ is natural Houston Black clay, which has been disturbed through the addition of construction debris (note obvious concrete rubble and rebar in foreground floor of trench). Conduits for electric lines protrude from the left trench wall in the foreground, and a PVC sewer line and conduits for electric lines cross the far end of the trench.

the absence of topographic breaks between these areas and Yanaguana Garden, this indicates that the fill that is so prevalent across the project area was not deposited atop an unmodified natural surface (i.e., the historic houses are not in "bowls" surrounded by higher areas of mounded fill). Instead, it appears that the project area saw repeated episodes of truncation of the surface and reworking of existing and introduced sediments to create a new surface that approximated the natural late-nineteenth-century one.

How many times this occurred is uncertain, but three major episodes can be projected. The first was when the Acequia Madre (principally the west wall) was demolished, perhaps early in the twentieth century. The second was in the mid 1960s when HemisFair '68 was built. And the third was at various times after 1968 when the HemisFair '68 temporary buildings were demolished and removed and modern improvements were put in place. 


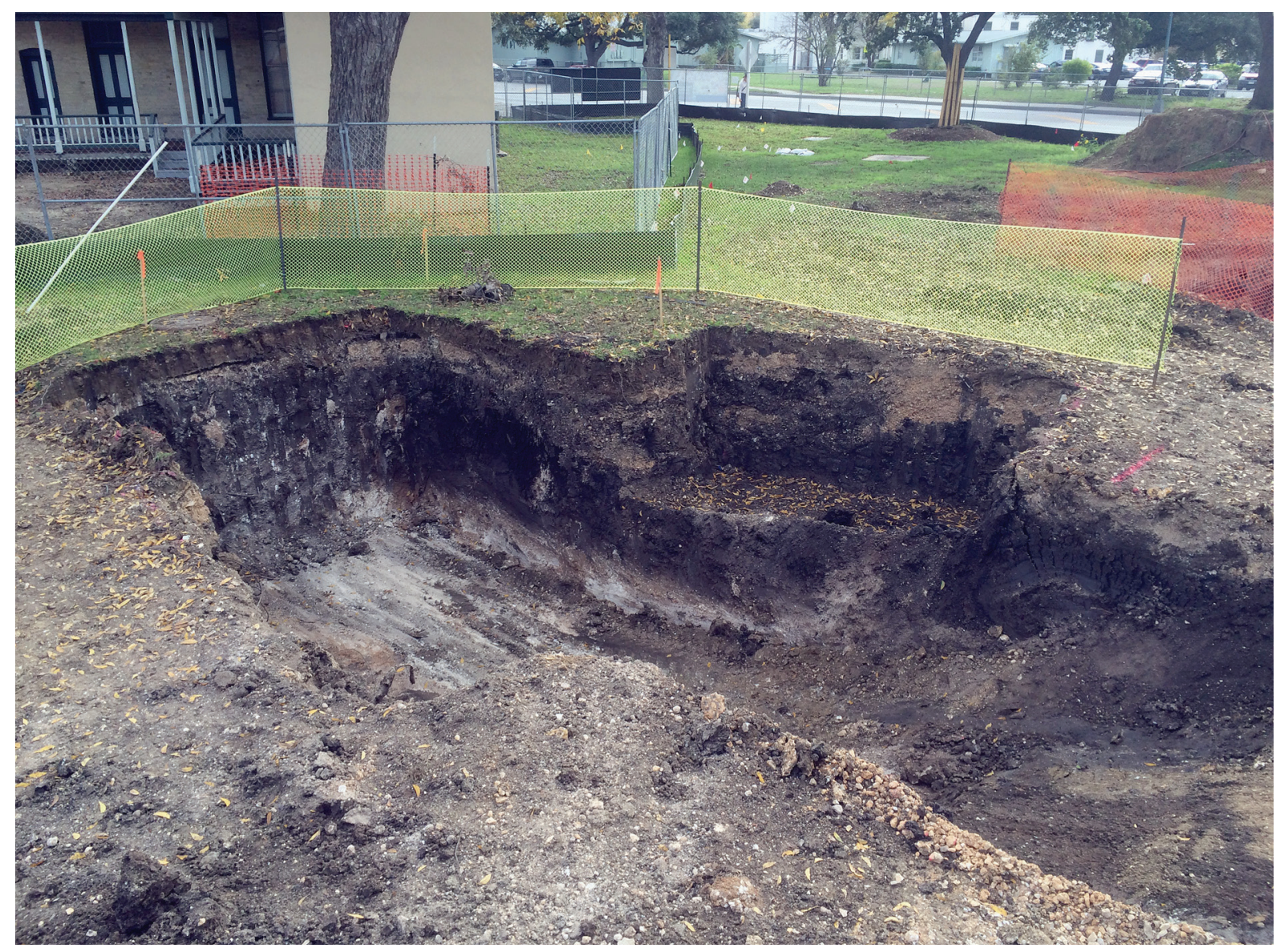

Figure 18. View to the southeast of intersecting sewer line bore pit and Bioswale Pit 3 showing subsurface stratigraphy (rear of the Koehler house is visible behind the excavations). The larger sewer line bore pit was a maximum of $8 \mathrm{ft}$ deep; it transected the shallower ( $5 \mathrm{ft}$ ) Bioswale Pit 3, the remnant of which is visible on the far side of the bore pit. At the left end of the bore pit, 1.5-2.0 ft of introduced fill (brown sediment above gravelly base material) overlies natural Houston Black clay, which has been churned by placement of a sewer line (manhole cover is visible just left of the pit at lath with orange flagging) and other buried utilities. Austin Chalk bedrock was encountered at a depth of about $7 \mathrm{ft}$. Moving right toward Bioswale Pit 3 and in the far wall of that excavation, mixed introduced fill thickens to $4.5-5.0 \mathrm{ft}$ before thinning to 2.0-2.5 ft.

\section{SITE 41BX8, ACEQUIA MADRE}

Four trenches were excavated to search for intact segments of the Acequia Madre before construction began, and evidence for the acequia were found in two or three of them. In the northeasternmost trench (PAI-1), a line of shaped limestone blocks $0.5 \mathrm{ft}$ thick that appeared to be the bottom of the east wall of the acequia was found at a depth of 2.6-2.8 ft (Figure 19). Three stacked rocks that lined up with the rocks in the trench bottom were visible in the south wall, starting at $3.0 \mathrm{ft}$ below the surface. There were no rocks indicating the west acequia wall. Obvious introduced fill (brown clay loam above reddish yellow gravelly clay loam visible in Figure 19) was present to a depth of about $2.3 \mathrm{ft}$ east of the wall remnant, above dark in situ sediment. Just west of the line of limestone blocks, clearly introduced fill (including a thick zone of white caliche containing construction rubble visible 
on the left and right sides of Figure 19) was present to a depth of $4.3 \mathrm{ft}$. Below that was $1.1 \mathrm{ft}$ of dark clayey sediment with abundant artifacts (glass, ceramics, metal); this sat on caliche bedrock at $5.4 \mathrm{ft}$ and appeared to be dirt used to intentionally fill the ditch in the early 1900 s.

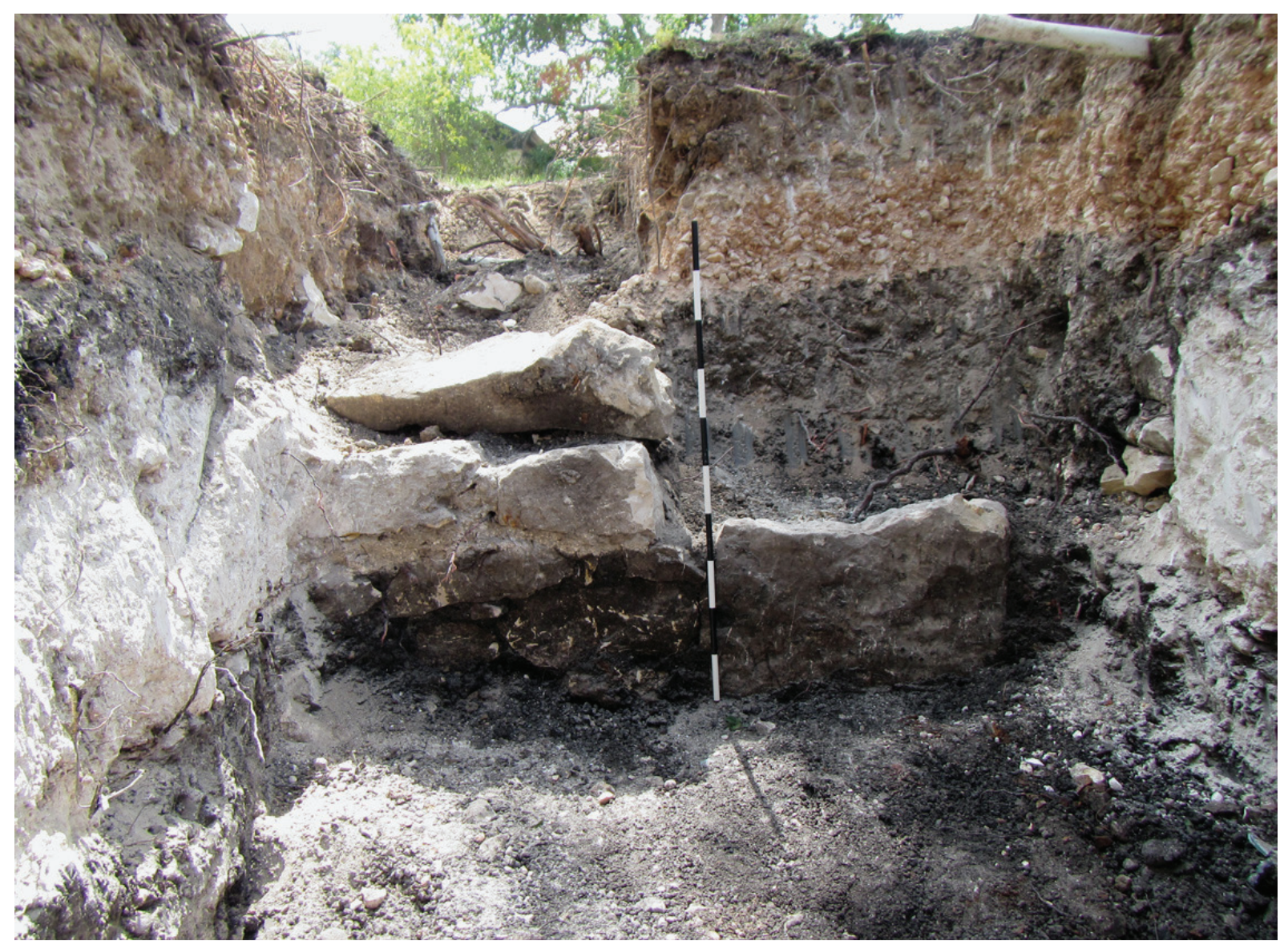

Figure 19. View to the southeast of the west face of the acequia wall segment in Trench PAI-1. The rock to the right of the meter-long photo scale and the two lower ones to the left of it are the intact vertical wall segment. The upper rock on the left is displaced from the wall.

The second trench (PAI-2) exposed thick twentieth-century fill and did not find any acequia walls. It was not recorded in detail because a broken water line caused the trench to partially fill with water, but the stratigraphy consisted of disturbed fill down to about $5.2 \mathrm{ft}$ over the full length of the trench, with possible caliche bedrock below that to at least $8.2 \mathrm{ft}$.

The third trench (PAI-3) had three horizontal layers of introduced modern fill to a depth of $4.6 \mathrm{ft}$ along its full length (Figure 20). Below that were two zones of probable acequia ditch fill at 4.6-6.6 ft inset into in situ dark sediments on the east side (at $4.6 \mathrm{ft}$ ) and what appeared to be caliche bedrock on the west side (at $5.2 \mathrm{ft}$ ). The upper zone of ditch fill (4.6-5.2 ft) clearly was cultural, as it contained historic 
trash such as metal and glass; it appeared as a lens about $6 \mathrm{ft}$ long. The lower zone lacked artifacts but looked like redeposited natural sediments; at its east edge at the bottom of the trench were several limestone rocks that could be the remnant of the east acequia wall, although they did not look like intact courses of rocks.

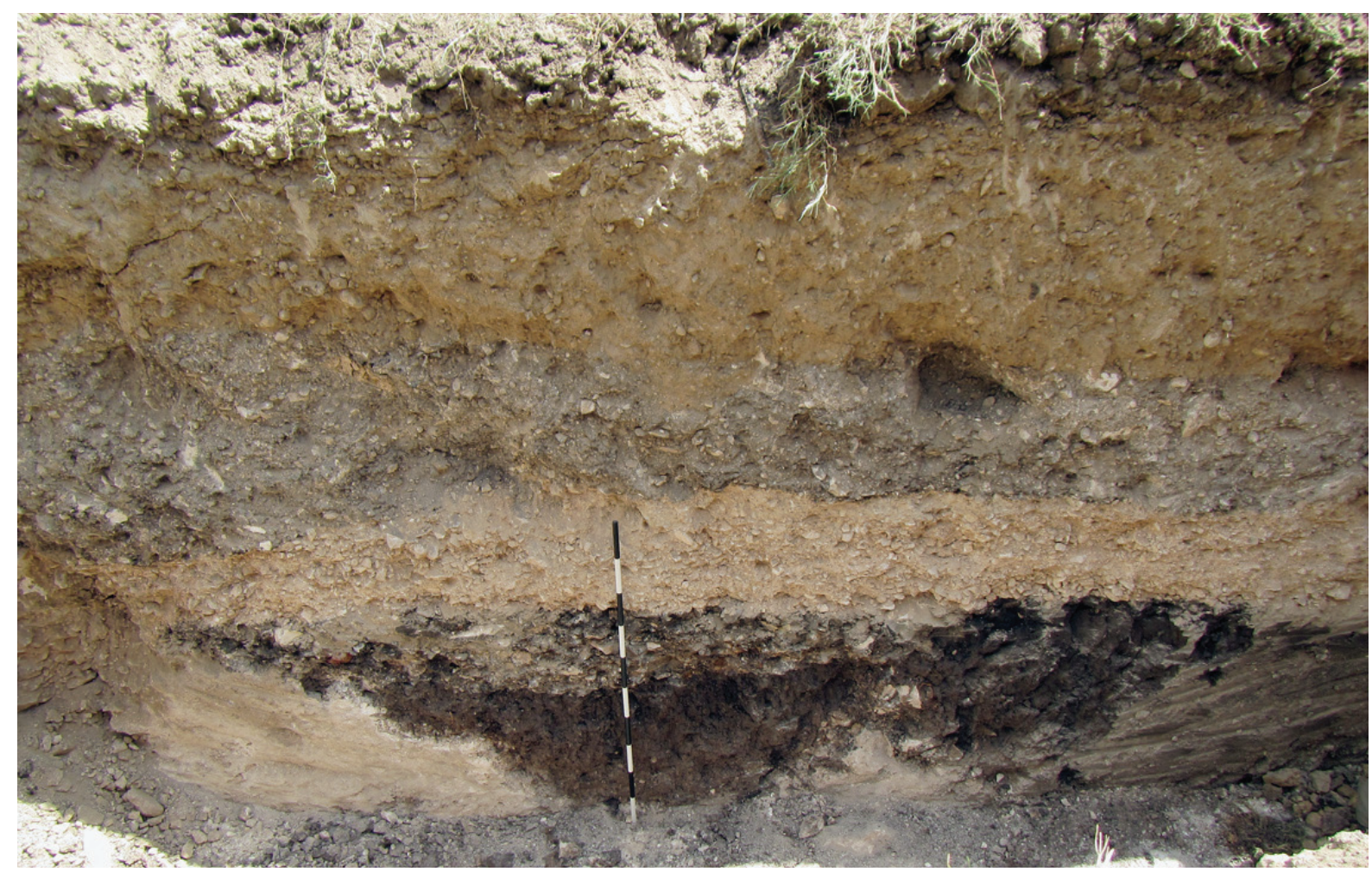

Figure 20. View to the northeast of the north wall of Trench PAI-3. The lower part of the meter-long photo scale is in front of probable lower acequia ditch inset into in situ black clayey sediment to the right and caliche bedrock to the left. The upper part of the photo scale is in front of the lowermost of three zones of introduced fill.

The fourth trench (PAI-4) exposed a segment of intact lower east wall of the acequia (Figure 21). The wall was $1.3 \mathrm{ft}$ thick, and the top of the intact segment was 3.0-3.3 ft below the surface. The west face of the wall was not fully exposed, but the southernmost rock that was exposed extended about $2 \mathrm{ft}$ vertically. Probing suggested that there are no more rocks beneath it, so what remains likely is the lowermost course of rocks. The backhoe removed abundant limestone rubble probably representing displaced pieces of the west wall and the upper east wall. This was especially evident in the south wall of the trench, where rubble was common for a distance of $4.9 \mathrm{ft}$ west of the intact wall segment at depths of 1.0-4.3 ft. Two of the more-intact rubble pieces were from large shaped blocks that were 1.6-2.0 ft across. The north wall of the trench showed two horizontal zones of fill to a depth of 1.3-1.6 ft, a wedge of caliche fill above and flanking the acequia wall to a maximum depth of about $3.3 \mathrm{ft}$, a zone of dark gray clayey fill to a depth of about $5.2 \mathrm{ft}$ where the acequia ditch likely was, and probable caliche bedrock below $5.2 \mathrm{ft}$. The dark gray 
clay contained rock rubble and scattered artifacts throughout, but it had a distinct lens of historic trash (bricks, glass, wire, other metal, etc.) at depths of 3.3-3.6 ft for a distance of ca. $3.6 \mathrm{ft}$ just west of the intact wall segment. As in the other trenches, the dark gray clayey sediments appeared to be mostly natural deposits that were moved around when the west wall of the acequia was taken out and the area filled and leveled (pre-HemisFair '68) and when the area was leveled again in association with HemisFair '68.

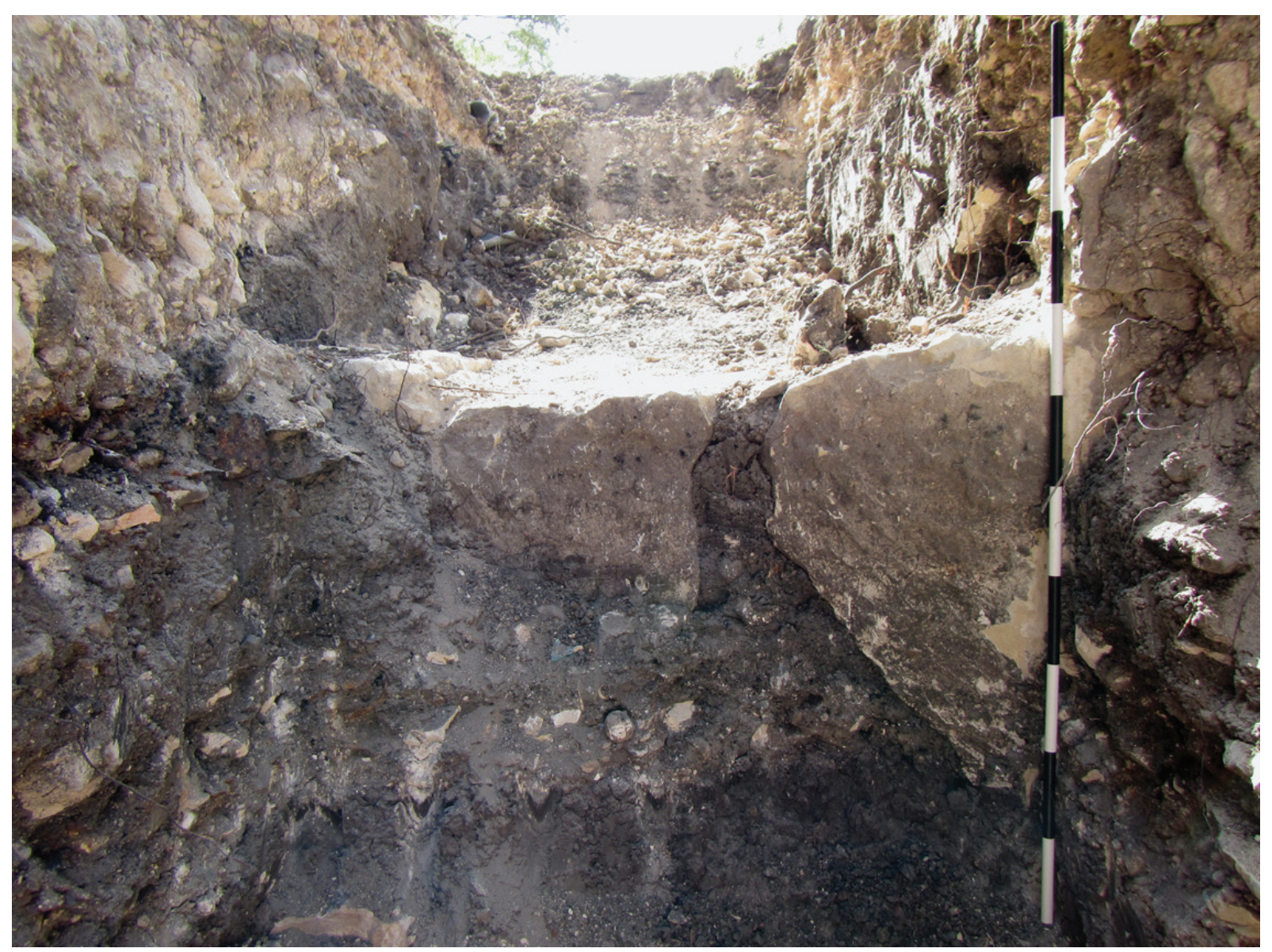

Figure 21. View to the southeast of the west face of the acequia wall segment in Trench PAI-4. Only the rock on the right is fully exposed. The lower portion of the rock on the left (middle of the trench) is obscured by ditch fill. Introduced fill above the vertical rocks is obvious in both trench walls.

Historic and modern artifacts were observed in all four trenches. These mostly consisted of nondiagnostic pieces of glass, metal (e.g., nails, rebar, possible furniture hardware, and flat can fragments or sheet metal), whiteware, stoneware, machine-made bricks, butchered animal bones, ceramic tiles, sewer pipes, and plastic. The more-diagnostic items, found in Trenches PAI-1, PAI-2, and PAI-3, consisted of the following: an annular-decorated ceramic sherd (probably dating ca. 1840-1900); and eight bottles with hand-tooled finishes and mold seams obliterated over the mouth and lip (predating 1903). The latter consist of a small cobalt blue glass bottle; a green wine bottle neck and mouth; two brown beer bottle necks; a clear medicine 
bottle neck and mouth; a large opaque bottle mouth similar to a large milk bottle; a clear glass medicine bottle with an embossed label that reads "Alamo Drug Store, A. Driess, San Antonio, Tex."; and a very small clear glass bottle embossed with "Ayer's Pills, Lowell, Mass.", which likely dates to ca. 1853-1870.

During construction, two additional trenches (PAI-5 and PAI-6) were excavated across the alignment of the acequia where two new storm sewer lines would intersect it. Trench PAI-5 was $9 \mathrm{ft}$ long, $2 \mathrm{ft}$ wide, and $6 \mathrm{ft}$ deep, and Trench PAI- 6 was $12 \mathrm{ft}$ long, $3 \mathrm{ft}$ wide, and $6 \mathrm{ft}$ deep. No evidence of rock walls or the acequia ditch was found, and few artifacts were observed in what was clearly mixed twentieth-century fill. Materials included whiteware fragments, glass bottle fragments, and a single corroded axe head, along with large pieces of concrete rubble, rebar, and plastic, including a Bill Miller BBQ cup at $5 \mathrm{ft}$ below the ground surface. These trenches, between Trenches PAI-2 and PAI-3, supported the conclusion reached during initial trenching that the acequia in this area had been heavily disturbed or destroyed.

Site 41BX8 is a Registered Texas Historic Landmark that is listed in the Historic American Engineering Record and that is shown as eligible for listing in the National Register of Historic Places in the Texas Historical Commission's Archeological Sites Database. The investigations reported here support this eligibility assessment, as well as the conclusion that the site warrants designation as a State Antiquities Landmark.

\section{SITE 41BX2068, TRASH PIT}

\section{Investigation and Description}

While trenching along a new sewer line route in the very southern portion of the project area, a basin-shaped pit containing historic artifacts was encountered and subsequently excavated; this feature was given the trinomial designation 41BX2068 (see Figure 9). Caliche base fill at this location was $2.5 \mathrm{ft}$ thick, and the feature was identified in the south wall of the trench at the contact between the fill and the underlying in situ black clayey sediments (Figure 22). Numerous animal bones and ceramic and glass sherds were observed in this profile, along with two main ash lenses. Based on this profile, the pit appeared to be ca. $4.5 \mathrm{ft}$ in diameter and to extend ca. $2.9 \mathrm{ft}$ below the contact between caliche fill and in situ sediments. Its full original vertical extent is unknown, since the original ground surface had been truncated, but it likely was no more than $5.4 \mathrm{ft}$ given the thickness of the fill above it.

A strategy for investigating this feature was developed in consultation with CoSA's City Archaeologist. It consisted of mechanically removing the introduced fill above the feature in the south wall of the trench and manually excavating a 1x1-m unit in the west part of the feature below that. The unit was excavated to a depth of $1.3 \mathrm{ft}$, at which point the western and southern pit margins became obvious; below that, only the pit fill was excavated to a maximum depth of $2.6 \mathrm{ft}$. The portion of the pit exposed in this excavation measured $3.0 \mathrm{ft}$ north-south by $2.8 \mathrm{ft}$ east-west. This left perhaps as much as $1.7 \mathrm{ft}$ of unexcavated pit to the east, 


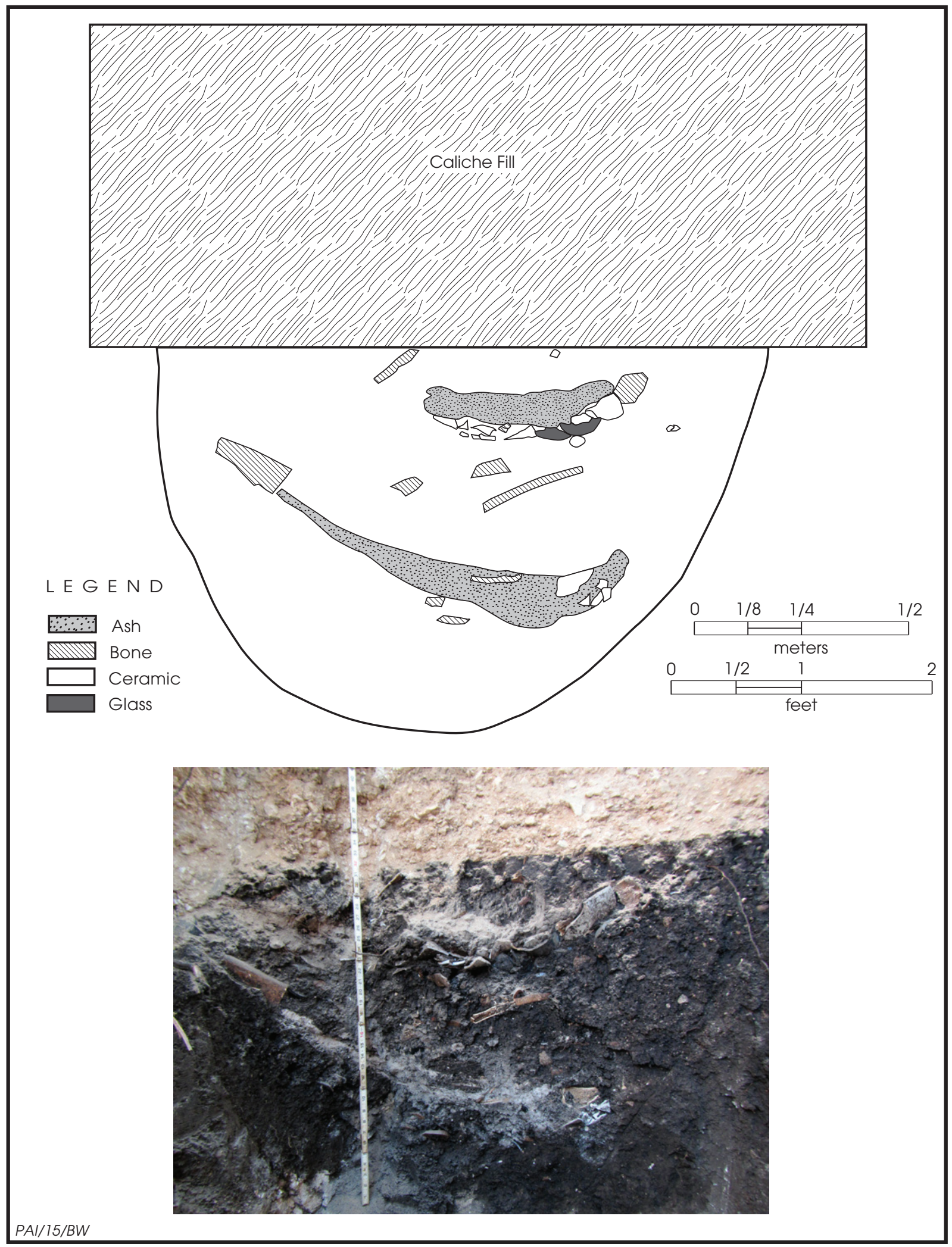

Figure 22. Profile and photograph of the south wall of the storm sewer line trench in which trash pit feature at 41BX2068 was found. 
judging from the original profile. Although the original profile indicated that the pit contained multiple lenses of fill, the excavation was done as a single vertical provenience rather than in levels; this was done because time for excavation was limited and there were no obvious differences in the content of the fill lenses in the pit profile. Another concession to the time shortage was that the clayey pit fill was not screened; the fact that artifacts were very abundant and easily removable by hand as the fill was troweled through also contributed to this decision.

Excavation of the 1x1-m unit, and its east wall profile showing the remaining unexcavated part of the feature (Figure 23), confirmed that the pit contained layers of household refuse, with several artifact layers capped by layers of wood ash and charcoal perhaps from fireplace cleaning. Because the pit was full of broken everyday household items such as glass food and drink bottles, ceramic drink bottles, ceramic dinnerware, drinking glasses, and animal bones, it is clear that this is a domestic refuse assemblage. Supporting this interpretation is the location of the pit along the back of an old city lot just 12-15 ft east of the Alamo Acequia (see Archival Research below). Trash was likely discarded some distance from the house (which faced onto Water Street to the east) to prevent foul smells and keep scavenging animals at bay. There is no way of knowing for certain if the pit was intentionally dug to serve as a trash receptacle, but a case is made below that it was, based on the absence of other better interpretations of the pit's function.

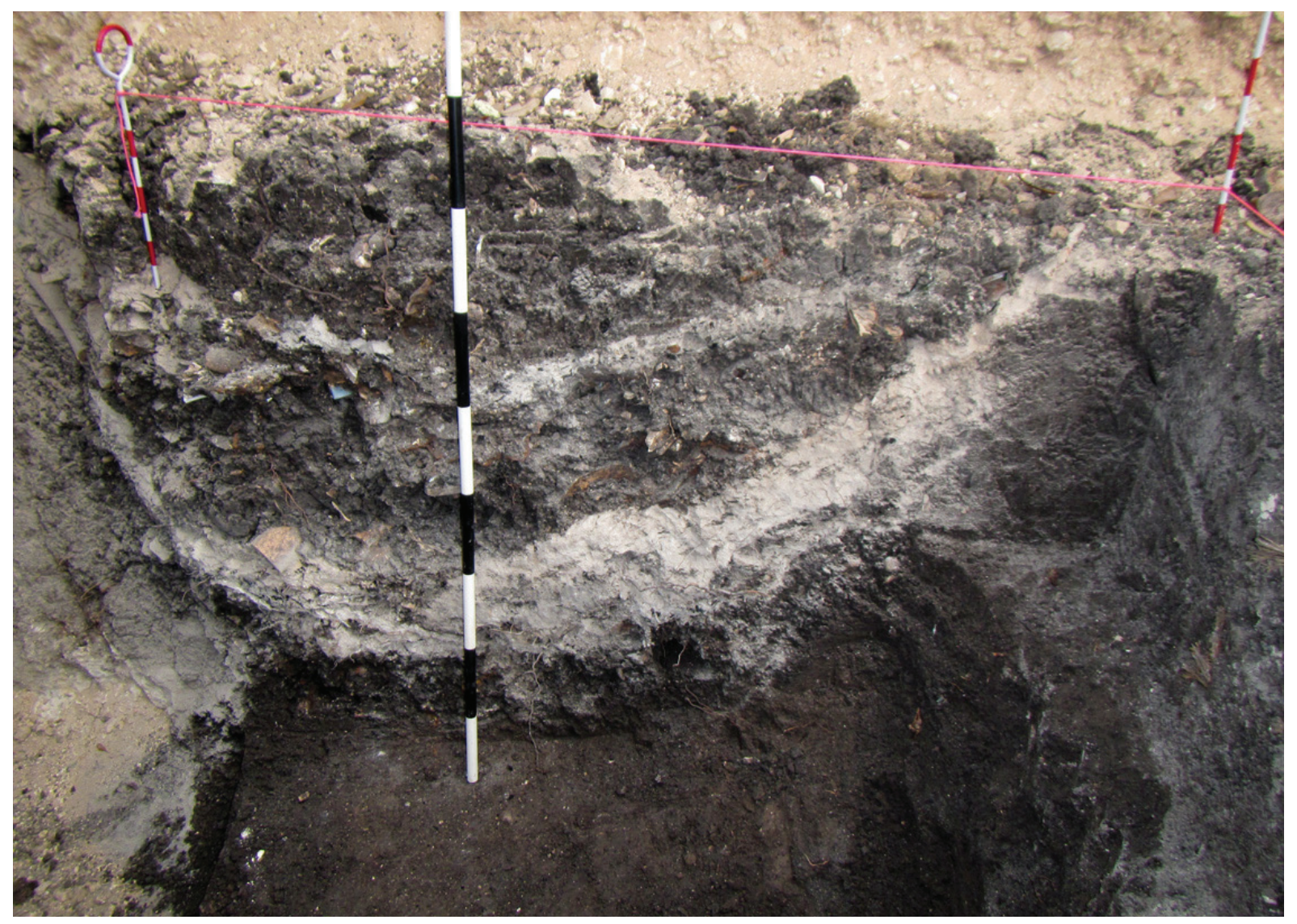

Figure 23. View of the east wall of the 1x1-m unit excavated into the 41BX2068 trash pit. Two main layers of charcoal-rich ashy fill are sandwiched between layers of artifact-rich dark clayey fill. 


\section{Materials Recovered}

Excavation of the pit feature resulted in a collection consisting of 1,369 artifacts. In describing these materials, the functional classification system used by Boyd et al. (2015) is followed. It organizes artifacts based on their function and use rather than their physical attributes. Artifact categories represented are kitchen/ household artifacts, clothing-related items, architectural artifacts, personal items, activities-related artifacts, faunal remains, lithics, and unidentifiable artifacts.

\section{Kitchen/Household Artifacts}

\section{FOOD STORAGE AND PREPARATION}

The artifacts most representative of this category are the many stoneware ginger beer bottles. Twenty-seven bottles can be identified using the bottle necks/ mouths as indicators of individual specimens. There are two types of bottles. One is a Bristol-glazed stoneware with brown slip, and the other is a green, alkalineglazed stoneware. Twenty-two bottles are Bristol glazed, and two bottle types are present: rounded shoulders with rounded body, and cylindrical body with an abrupt break at the shoulders. Bristol is a smooth white glaze that originated in Bristol, England, and became a popular treatment for stoneware during the Victorian period as the public's interest in health and sanitation grew, with white being a sign of cleanliness (Greer 1981:210). Prior to 1920, this glazing technique was used in combination with a brown slip on the upper portions of vessels and was purely decorative (Greer 1981:212). The 22 Bristol-glazed bottles display this two-toned pattern. Only 1 bottle could be completely reconstructed, and it measures 7.25 inches tall and 2.875 inches in diameter at the base (Figure 24a). The approximate volume of this vessel is 20 fluid ounces. The manufacturer marking of Powell Bristol is stamped on its side at the base. William Powell invented the popular Bristol glaze in 1835, and his pottery manufactured ceramics with the impressed mark of Powell Bristol until it was sold to Price, Sons \& Co in 1906 (Godden 1991:509). Therefore, this bottle dates between 1835 and 1906. Another bottle contains a single "W" marking on its side at the bottom, but the manufacturer remains unknown. In addition to the 22 bottles, 59 Bristol-glazed fragments were recovered.

The other five ginger beer bottles are green, alkaline-glazed stoneware. The one that could be reconstructed bears the label "Copeland's Celebrated Ginger Pop" stamped into the surface (Figure 24b). It measures 7.5 inches tall and 3 inches in diameter at the base and is slightly flared at the shoulders. The approximate volume is 24-28 fluid ounces. Stamped label fragments on sherds not belonging to this reconstructed bottle indicate that at least four other Copeland's bottles are represented. No information could be found regarding this product. In addition to the 5 bottles, 22 fragments from Copeland's bottles were recovered.

A single stoneware mineral water bottle was recovered. It could not be reconstructed, nor does it have a maker's mark. It consists of two fragments, the base and the mouth. 


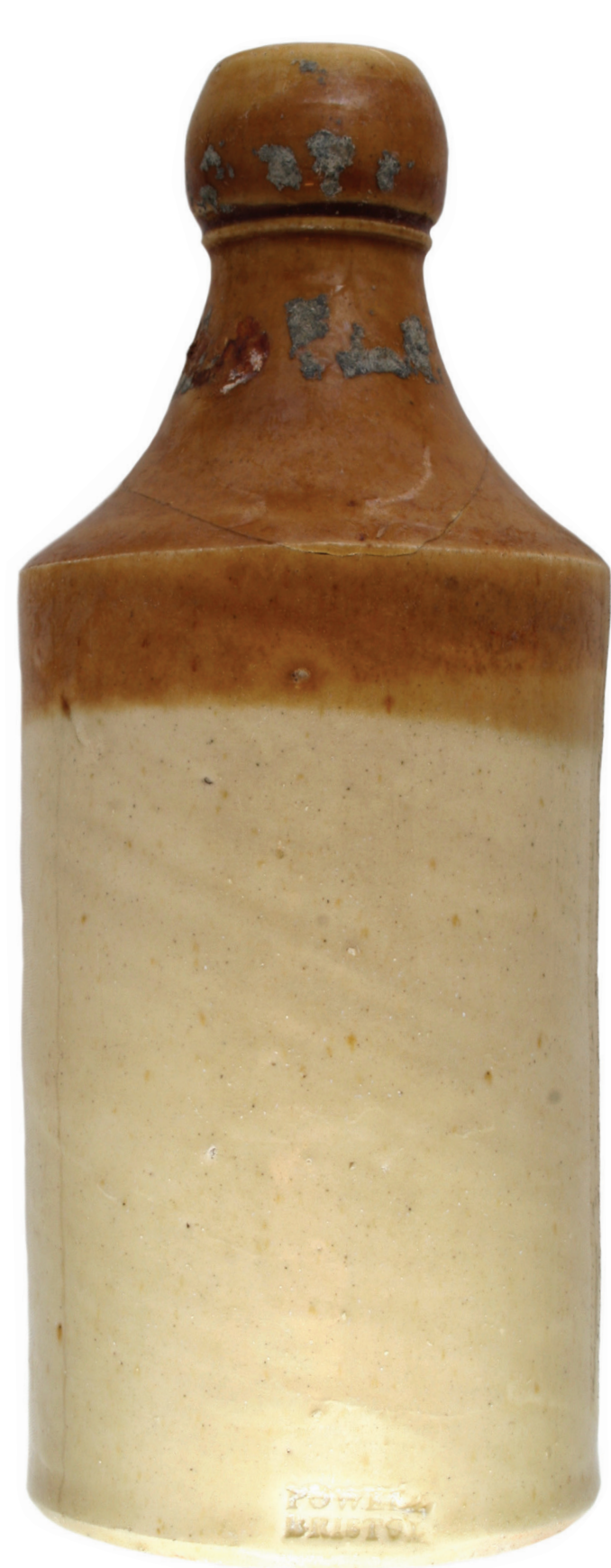

a
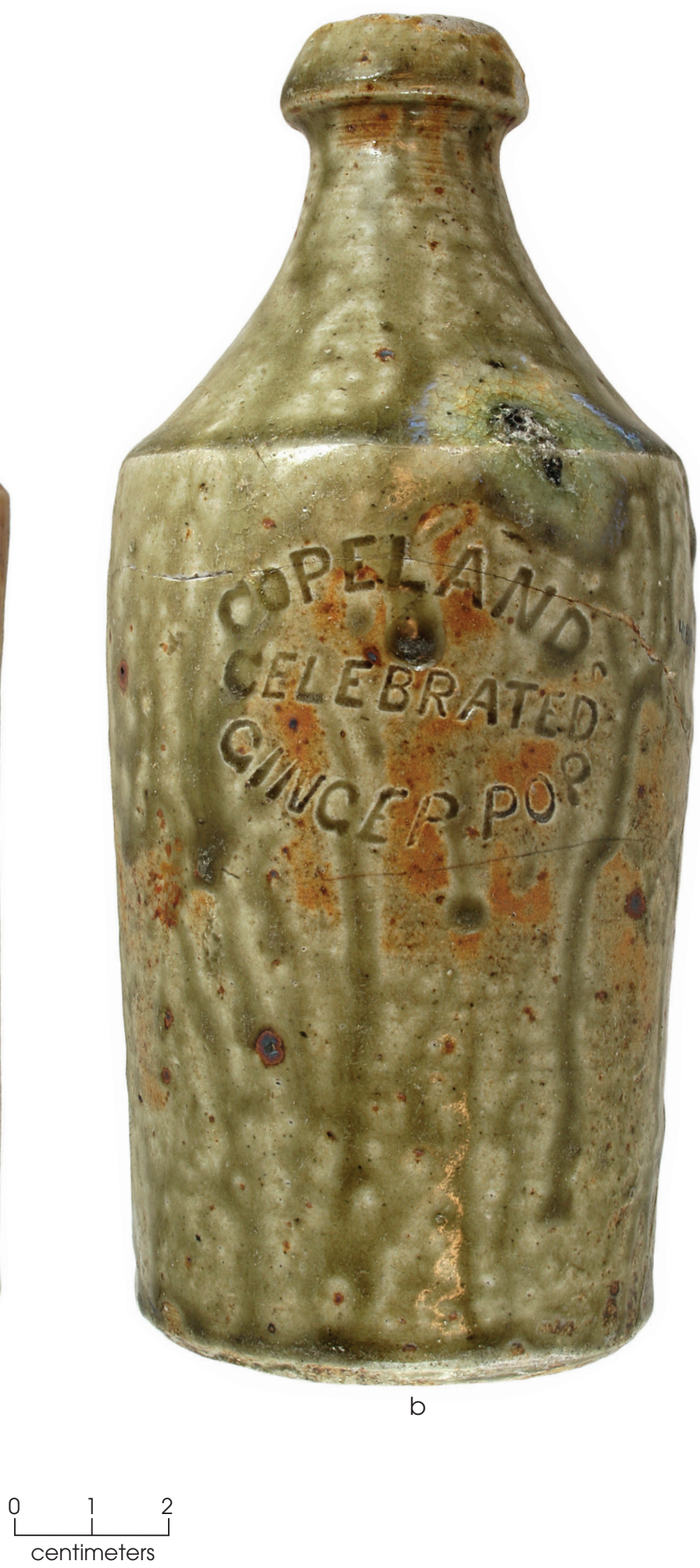

Figure 24. Examples of the two styles of ginger beer bottles recovered from the 41BX2068 trash pit. (a) Powell Bristol-glazed bottle; (b) Copeland's Celebrated Ginger Pop bottle. 
Another artifact in this category is a partially reconstructed clear glass bottle base of Lea \& Perrins Worcestershire sauce. The small glass stopper was also recovered. Only the bottom half of the bottle remains, and the embossed letters of Lea \& Perrins are still visible. The stopper is also labeled the same. Both pieces show heavy patina and no obvious markings that allow this bottle to be dated.

\section{FOOD SERVICE AND CONSUMPTION}

This category of household artifacts is dominated by whiteware sherds. Several different varieties of dinnerware, as well as different makers, are represented in the collection. At least 18 individual whiteware vessels were identified, consisting of 6 saucers, 5 cups, 4 plates, 1 bowl, 1 plate/saucer, and 1 pitcher. Each of these vessels was partially reconstructed. In addition to the vessels, 118 fragments of whiteware were recovered.

Eight makers' marks were observed on the examples of whiteware collected. Of these, five are identifiable in terms of manufacturer, and three can be confidently dated. A reconstructed bowl is marked "Ironstone / J.Wedgwood / China" on the base (Figure 25a); it was manufactured by John Wedge Wood between 1841 and 1860 (not to be confused with the famed Josiah Wedgwood; Godden 1991:656, 687). A saucer base bears the impressed mark of "JAMES EDWARDS / DALEHALL" (Figure 25b); it was manufactured by James Edwards \& Son sometime between 1851 and 1882 (Godden 1991:230, 231). The last datable mark is that of Pinder, Bourne \& Co., and it is found on the base of a whiteware pitcher and the base of a saucer. The design is printed and consists of an eagle with a shield and banner and labeled "IMPERIAL" above the eagle and "WHITE GRANITE" below it (Figure 25c). These ceramics were manufactured between 1862 and 1882 (Godden 1991:495).

All four of the whiteware plates have marks from William Adams \& Sons. This printed design is an eagle with a shield clutching arrows, something very American, and labeled "IMPERIAL" above the eagle and "FRENCH PORCELAIN" below it (Figure 25d). A date of manufacture other than mid-nineteenth century could not be found. Godden (1991:21) states that this mark may have different variations and designs, with the name of the individual pattern also included. Another mark from a single fragment of whiteware was that of W. Baker (Figure 25e). Since other defining attributes of the design are missing, it can only be dated to the broad timeframe of 1839-1932 (Godden 1991:51).

One fragment of whiteware has the common British Royal Arms mark and postdates 1837 (Figure 25f). The arms consisted of an oval shield with a lion on the right and unicorn to the left. In 1837, the shield was divided into four quadrants (Kovel and Kovel 1986:267). This sherd has no other attributes to allow it to be dated more precisely. Two other whiteware sherds have nondiagnostic marks. A plate or saucer fragment has the word "Warranted" stamped into the base (Figure 25g). Another sherd has raised letters that are too small to read (Figure 25h).

At least six other decorative ceramic styles and vessels were recovered. One is a hand-painted teacup with no maker's mark (Figure 26a). Other fragments are also from teacups, one with a black stripe around the rim, and the other with a 

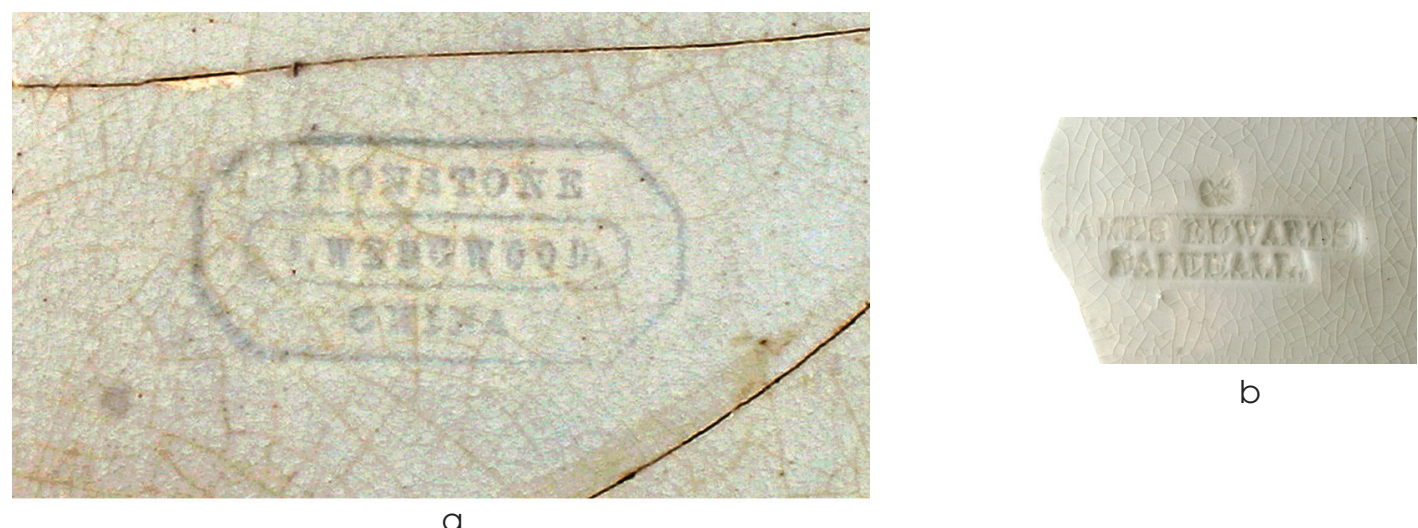

b

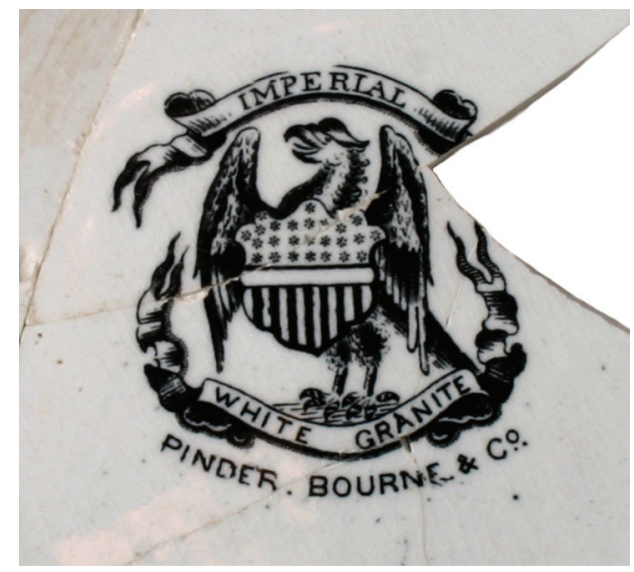

C

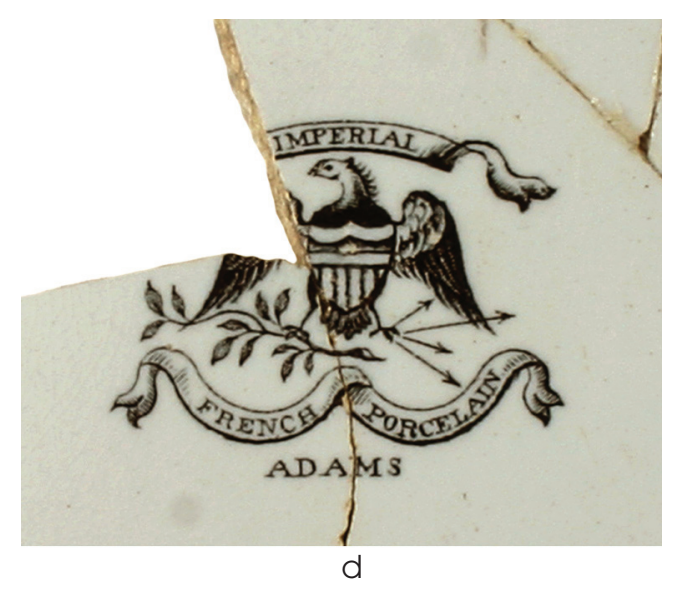

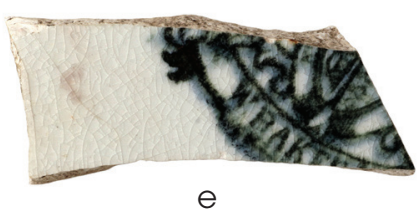

e

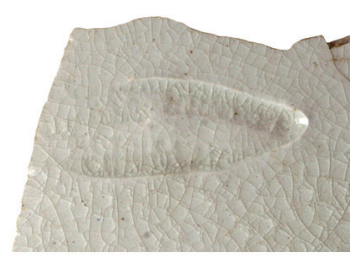

h

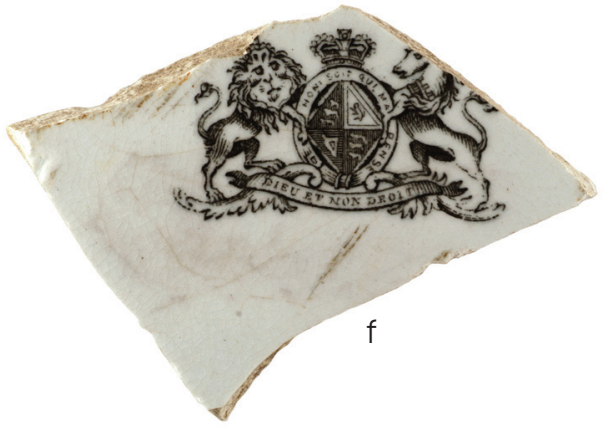

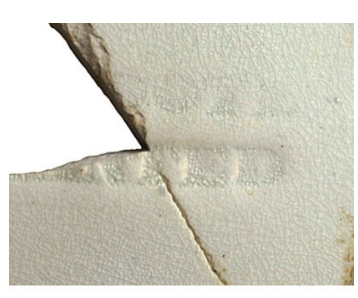

g

Figure 25. Whiteware fragments with makers' marks from the 41BX2068 trash pit. (a) John Wedge Wood maker's mark; (b) James Edwards \& Son maker's mark; (c) Pinder, Bourne \& Co. maker's mark; (d) William Adams \& Sons maker's mark; (e) W. Baker maker's mark; (f) British Royal Arms mark; (g) unidentified maker's mark stamped "WARRANTED"; (h) maker's mark with letters too small to read. 
brown stripe around the rim. A fragment of a teapot lid has thin red and blue lines as well as a thicker black line around it (Figure 26b). A total of nine annular ware fragments are present, primarily with light blue and black banding (Figure 26c-e). Annular wares generally date between 1840 and 1900 in the United States (Ramsay 1939:149). Lastly, the only transfer-printed ceramic is a teacup broken into five pieces. The design is a blue or gray tree/vine pattern, with the word "Fibre" printed across the bottom indicating the "Fibre" pattern (Figure 26f, g). No manufacturer could be identified.

Five partially reconstructed clear glass tumblers are in the food service and consumption category (Figure $26 \mathrm{~h}$ ). Fragments with at least one side intact measure 3-4 inches tall. All of the tumblers are similar in design and size, but they differ slightly in thickness and weight. Ten fragments could not be attributed to any of the five individual vessels.

Two iron knife fragments are in the food service and consumption category. One is a partial blade fragment with a portion of the tang still attached. The tip of the blade has been broken, and what remains is covered in rust. The other fragment is the tang. It is also rusted, but two brass pins are present, and these would have held the handle in place.

\section{FURNISHINGS}

Home-furnishing artifacts consist of just a few items. Two are small, corroded, and fragmentary iron items. One is a small diamond escutcheon, likely from a trunk, dresser, or similar piece of furniture. The fragment measures 2.5 inches long and 1 inch wide. When complete, the whole piece would have measured ca. 4 inches. The other rusted iron artifact is a segment of trunk strap, measuring 5 inches long and 1.25 inches wide. One small hole was observed where it was tacked to the trunk.

Another furnishing item is a heavy rusted fragment of a stove burner plate. It is too rusted to obtain an accurate measurement. The only other furnishing item is a single glass lamp chimney rim. Only the upper quarter inch is present.

\section{Clothing-Related Artifacts}

Most of the few clothing items are buttons (Figure 27). The breakdown of the 10 buttons is as follows: 2 bone, 3 white metal, and 5 white Prosser. All buttons have four holes and range in size from 18 to 30 line. The bone buttons are 24 and 30 line. The white metal buttons, which are identical, are 30 line. The white Prosser buttons are of two sizes: 18 line $(n=2)$ and 29 line $(n=3)$. The only other clothing items are a single brass rivet, possibly from a boot/shoe or pants/overalls, and a leather boot heel. The leather heel is small, indicating that it is from a child's shoe/boot.

\section{Architectural Artifacts}

Architectural artifacts consist of nails, wire, and hinges. A total of 47 nails were recovered from the pit, with 46 being cut nails and only 1 being a wire nail. Six wire fragments were recovered. The 2 hinges represent different styles, with one 

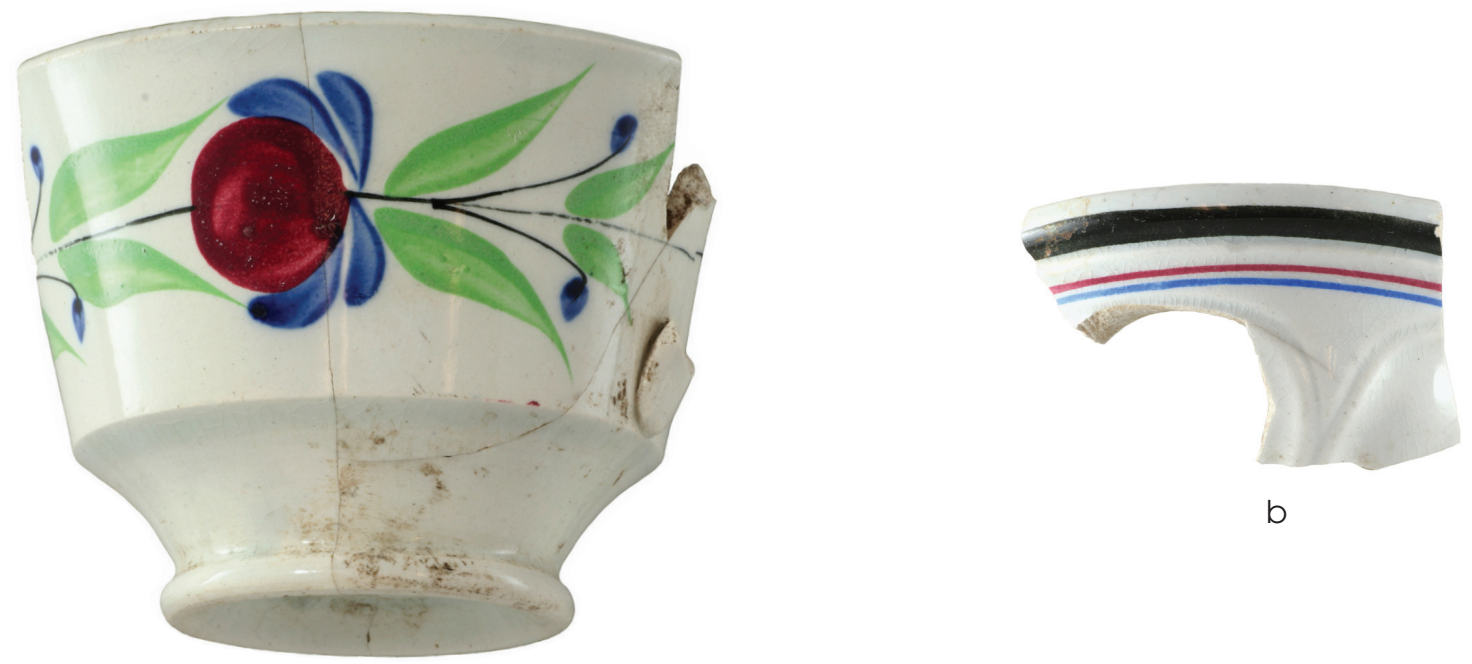

b
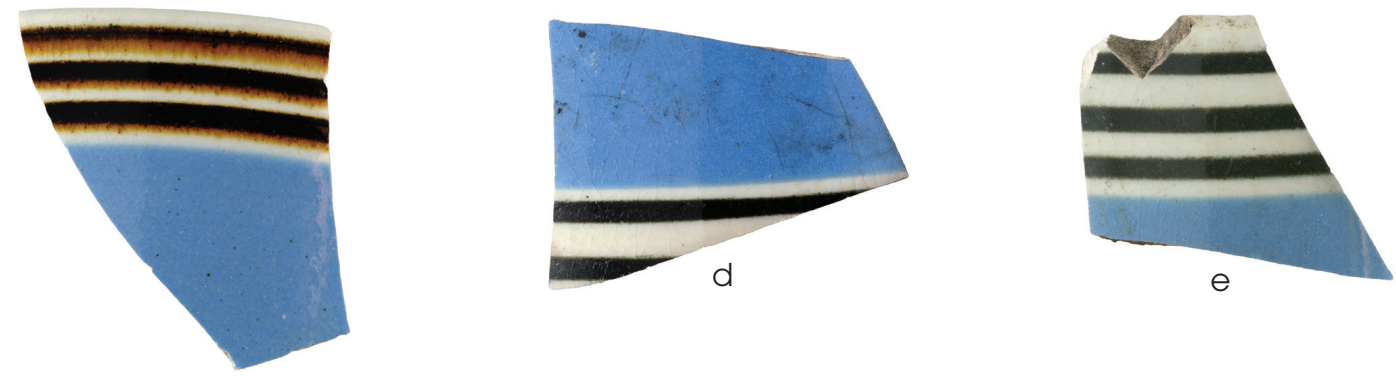

C
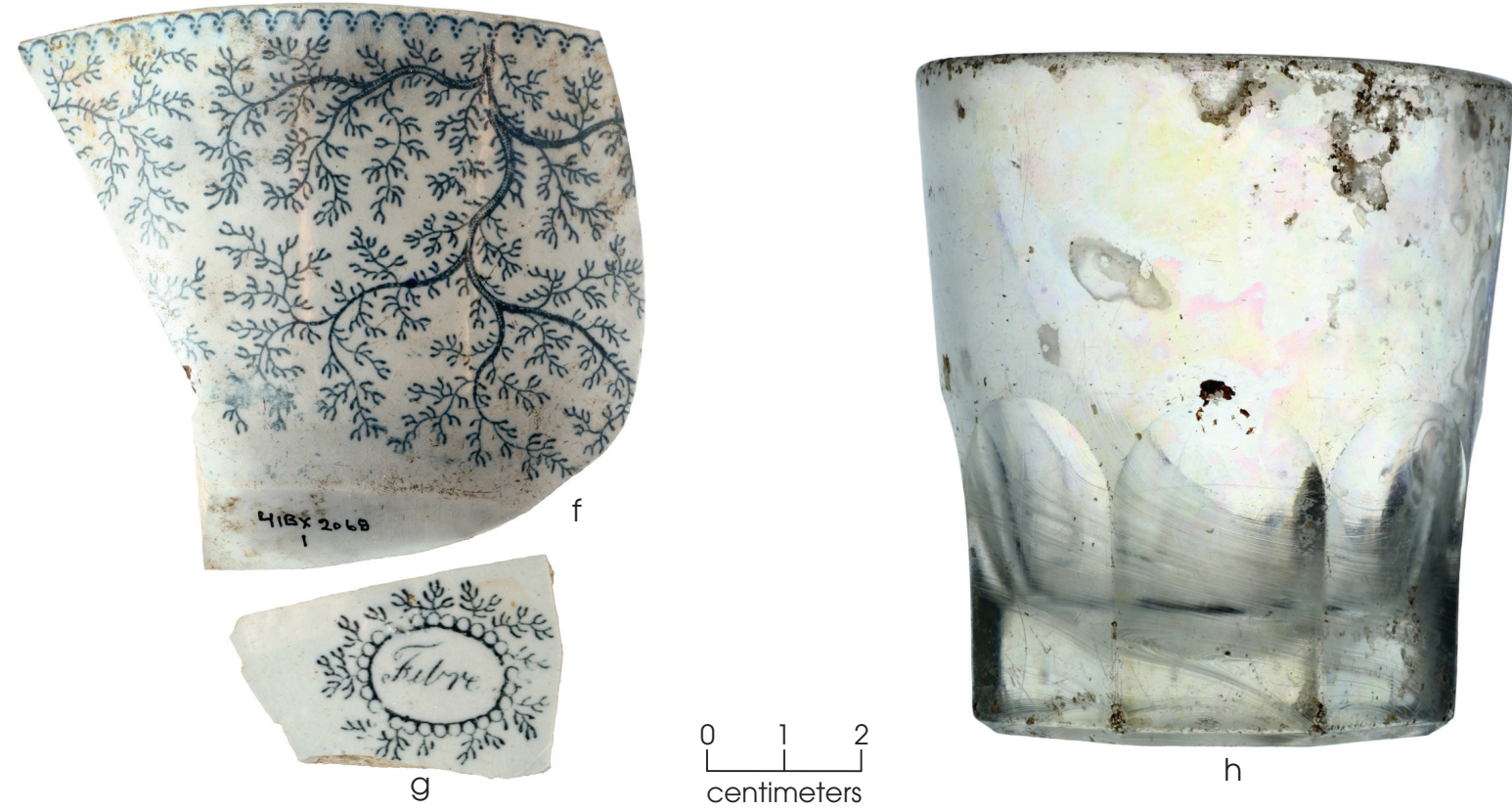

$\mathrm{h}$

Figure 26. Additional decorated ceramic styles and glass tumbler from the 41BX2068 trash pit. (a) Handpainted teacup; (b) striped teapot lid fragment; (c-e) annular ware fragments; (f, g) "Fibre" pattern transferware; (h) glass tumbler. 

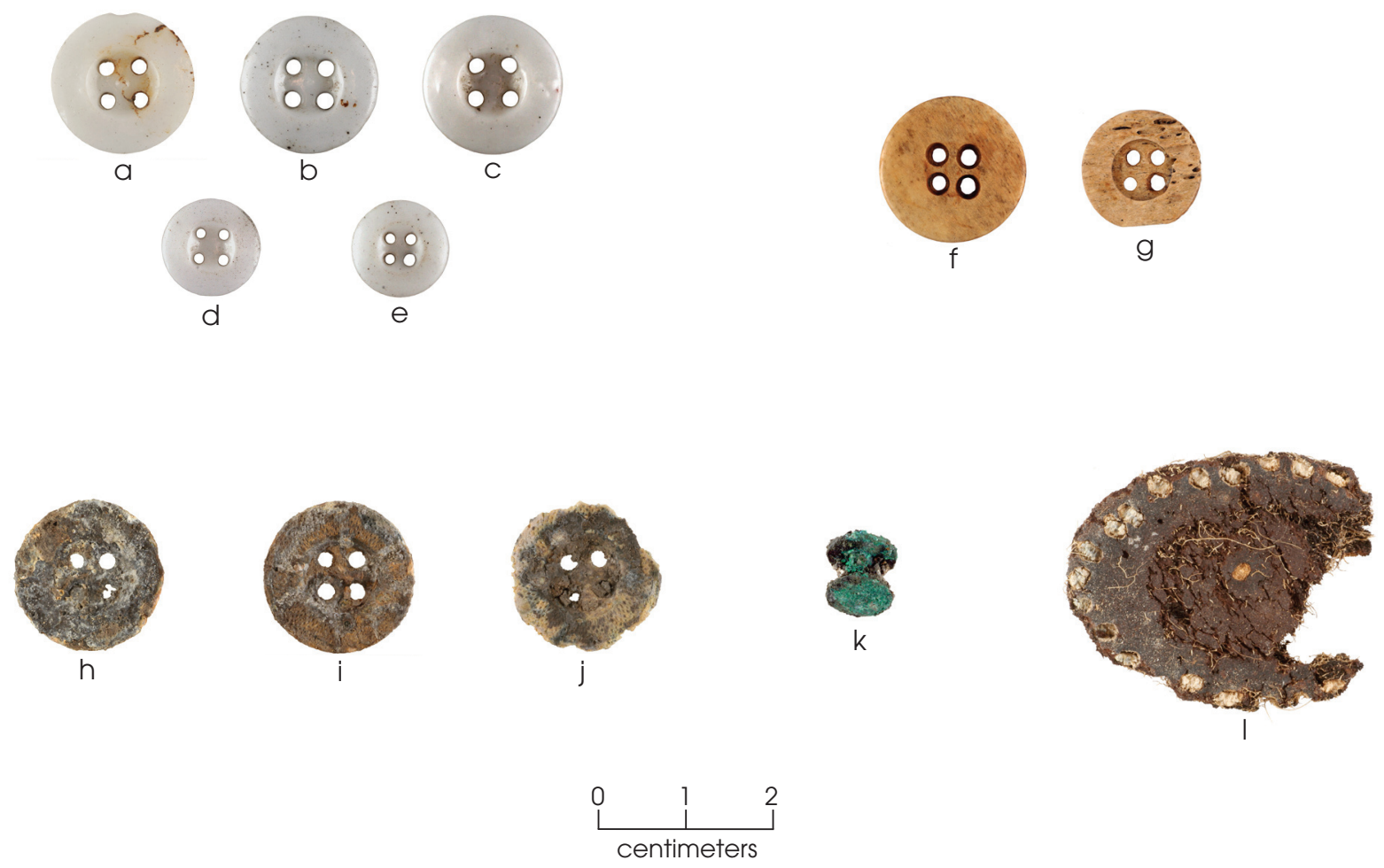

Figure 27. Clothing-related artifacts recovered from the 41BX2068 trash pit. (a-e) White Prosser buttons; (f, g) bone buttons; (h-j) white metal buttons; (k) brass rivet; (l) leather boot/shoe heel.

being a fragment from a strap hinge and the other being a gate hinge. The overall condition of these items is very poor, and they are heavily corroded with rust. Two small twisted segments of copper wire were recovered, but it is unclear what they belonged to.

\section{Personal Artifacts}

Glass bottles related to alcohol and medicine dominate this category, and the only accouterment recovered is a single folding pocketknife. The iron blades have rusted into one piece, but the white metal handles and bolstered ends are partially visible.

Four medicine bottles were identified. Two vessels are based on the presence of two clear bottle mouths and necks with flat/patent finishes. One is very small, and the finish is applied, while the other example is larger with a hand-tooled finish. A green bottle base has embossed lettering on its sides indicating that it held Udolpho Wolfe's Aromatic Schnapps. An advertisement in the Evening Post of Wellington, New Zealand, on June 21, 1877, indicates that this "medical stimulant" was a "curative of debility." The fourth example is a brown square bottle base that was partially reconstructed. Its shape suggests it was likely a panel medicine bottle. The only markings embossed on a side read "KY." 
Most of the glass bottles from the trash pit fill fall into the alcohol category, based on color, general base shape, and mouth/neck finishes. Using the bases alone, at least 19 individual alcohol bottles are in the assemblage. Bottle neck/mouth finishes are used to better describe the types of bottles and the products they held. It is possible that more are represented, but the many glass fragments make it difficult to accurately determine if there are additional individual bottles.

Six green glass bottles have applied champagne-finish necks with a small band of glass applied to the exterior of the neck. These likely held wine or champagne. Five bottles have wine/brandy finish necks, 4 of which are dark green and 1 of which is brown. Three of the dark green ones have applied finishes, while the other green bottle and the brown one have hand-tooled finishes. The specific products within these bottles are unknown, but alcohol is likely. Four examples of ring/oil finish necks were found, with three being green and having applied finishes and one clear one having a hand-tooled finish. Once again, specific contents are unknown, but color and shape suggest alcohol. Two green double oil/mineral neck finishes are represented. Each has an applied finish. Lastly, a very dark green applied flat/patent finish is from a case gin bottle, and a green applied ring/oil finish is from a liquor bottle.

\section{Activities-Related Artifacts}

TOYS

This category is represented by a single ceramic doll head (Figure 28a). It appears to have been discarded when it broke at the neck. The head was that of a small girl doll, hand painted with dark hair, but most of the paint has deteriorated.

\section{WRITING}

Evidence for writing was found in the form of two writing slate fragments and a partial glass ink bottle. The former are plain slate that was likely framed with wood that has rotted away (Figure 28b). Both fragments appear to be corner pieces, with the larger one measuring 6 inches long and 2.5-3.0 inches wide, and the smaller fragment measuring 1 inch by 1.5 inches. The clear glass ink bottle consists of the base measuring 2.375 inches in diameter with a small segment of one side reconstructed. Sides of the bottle are faceted, with 10 total facets, but no markings are present.

\section{SEWING}

A pair of iron scissors is the only sewing-related item (Figure 28c). One half is complete and measures 4.5 inches long, and the other is missing the blade tip and part of one finger/thumb hole; both are heavily corroded.

\section{HORSE TACK AND HARNESS}

The most striking artifact in this category is brass spur fragment (Figure 28d). Measuring just over 2 inches wide across the arms, the spur is small and 


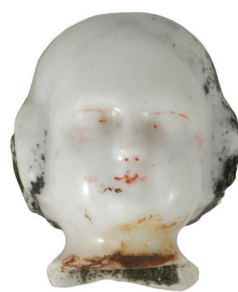

a
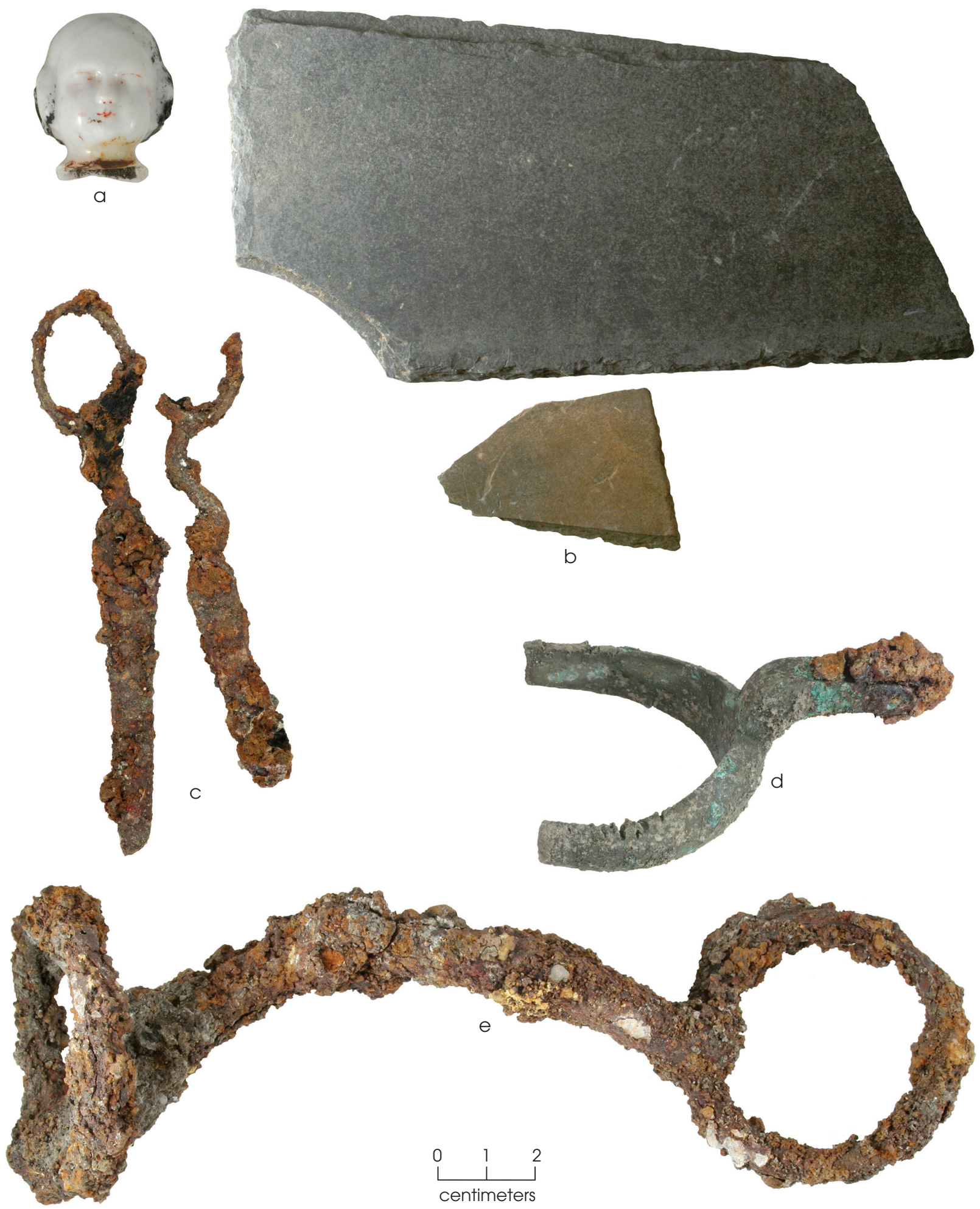

Figure 28. Activities-related artifacts recovered from the 41BX2068 trash pit. (a) Ceramic doll head; (b) writing slate fragments; (c) scissors; (d) child's brass spur; (e) snaffle bit. 
likely belonged to a child. Both of the terminals are broken from the arms, so it is not possible to determine how this spur fastened. Only a portion of the rowel is present. It is iron and much of it has rusted away.

A complete snaffle bit is heavily rusted such that the joint at the center of the bit is fused solid (Figure 28e). The rings on either end are also rusted in place and cannot be moved. Two rusted cinch rings were also found, one 2.25 inches in diameter and the other 2.5 inches in diameter.

The only harness hardware consists of two small iron roller buckles encrusted in a thick layer of rust. The smaller of the two buckles measures $1 \mathrm{inch}$ across and still has the tongue and roller, although they cannot move. All that remains of the other buckle is the 1.5-inch frame.

\section{Faunal Remains}

Appendix A contains a description and analysis of the vertebrate faunal remains recovered from the trash pit. The assemblage consists of 919 bones weighing $13.2 \mathrm{~kg}$. Almost two-thirds $(\mathrm{n}=581)$ are unidentified mammal bones. Most of the remainder are cow or likely cow $(n=270)$, with domestic pig being next most common $(n=31)$. Other identified animals represented by 1-3 bones each are domestic goat, domestic sheep, horse or donkey, white-tailed deer, and turkey.

\section{Lithics}

Recovered from the various layers of feature fill were 20 chert and limestone pebbles and cobbles ranging in size from 1 to 4 inches; many more were observed and not collected. They clearly were introduced into the feature fill, since these types of stones do not occur naturally in this setting. Some had been modified by flaking and appeared to be prehistoric chipped stone tools, but they obviously were in a context unassociated with Native American occupation. Many of the rocks were burned, and it is surmised they were used as heating stones in the fireplace of the house and discarded with ash and charcoal when the fireplace was cleaned out.

\section{Unidentifiable Artifacts}

This category consists of items that are too fragmentary, corroded, or small to be assigned to a specific artifact type. It includes 16 pieces of rusted iron that can be identified as banding fragments but not the actual type of banding (i.e., barrel banding, trunk straps, etc.). Other unidentifiable rusted iron items are 3 heavy fragments and 21 small pieces.

There are 293 glass bottle fragments that cannot be attributed specifically to alcohol, food, medicine, or other bottle types. By color, these consist of the following: green (all shades), $\mathrm{n}=230$; clear/opaque, $\mathrm{n}=55$; amber/brown, $\mathrm{n}=6$; and aqua, $\mathrm{n}=2$. Eleven of the green glass fragments contain embossed lettering, but the only thing that can be read is "New York" on a few fragments. 


\section{Chronology and Associations}

The ceramic artifacts, particularly the whiteware vessels with identifiable makers' marks on their bases, are most useful for determining the age of the feature. As stated earlier, eight different makers' marks are represented, five of which could be identified in terms of manufacturer, and three of which could be dated to when they were made: (1) John Wedge Wood (1841-1860); (2) James Edwards \& Son (1851-1882); and (3) Pinder, Bourne \& Co. (1862-1882). The last one indicates that the pit postdates 1862. Determining the last date of use is more difficult, since people can use ceramic vessels for generations following their period of manufacture, but the 1882 terminal date for two of the marks makes an estimate of 1880s-1890s seem reasonable.

The glass bottles recovered are consistent with the interpretation that the feature dates to the latter part of the nineteenth century, since none display characteristics of bottles made by an automatic bottling machine. Machine-made bottles were first manufactured in 1903 and became increasingly common after 1905 (Lindsey 2015).

Other temporally sensitive artifacts support the idea that the pit dates to the latter part of the nineteenth century, despite not being very diagnostic. The Powell Bristol stoneware bottle must predate 1906. Annular ceramic wares were manufactured earlier but are often found in 1840-1900 contexts. Following the creation of the Prosser process in 1840 (Sprague 2002:111), Prosser buttons flooded the market in the latter half of the nineteenth century, often replacing bone and other materials. The predominance of cut nails also points to the latter part of the nineteenth century, since wire nails were introduced in the 1890s and became the preferred choice only after 1900 (Wells 1998:87), as does the Udolpho Wolfe schnapps bottle with the 1877 newspaper advertisement.

Of course, the actual length of time the pit was used was much shorter than the several decades between 1862 and the end of the nineteenth century. Multiple episodes of dumping were evident in the pit profile, but it was not left open long enough for natural processes to contribute to filling it. Further, artifact densities were consistently high throughout the entire pit, and the various kinds of artifacts were highly mixed. For example, two of the Copeland ginger beer bottles and one of the Bristol-glazed ginger beer bottles were found at the top of the pit, and other examples of these kinds of bottles were commonly recovered throughout the excavation of the pit. Based on this evidence, it appears the pit may have been used for no more than a few months, and certainly for no more than a few years.

The archival research presented below indicates that the feature most likely relates to occupation by the Volpert family, or perhaps less likely, tenants. Minus time away during the Civil War, Marcellus Volpert lived on the property from 1856 till at or near his death in 1888 . He married in 1869 , but his wife died in 1879 , after which their four children attended school and lived elsewhere for at least part of the time. Between 1888 and 1899, the property may have been occupied occasionally by a family member, housed tenants, or been vacant at times. Between 1899 and 1944, when the lot passed out of the hands of Volpert family members, only tenants lived there. 


\section{Discussion}

The 41BX2068 pit is interpreted as a trash pit based on its contents (i.e., household refuse) and its position at the back of the lot away from the house location. To determine if similar kinds of features have been documented in the vicinity of Yanaguana Garden, reports on archeological work done for the Henry B. Gonzales Convention Center and the Alamodome nearby were reviewed. These investigations documented numerous house sites and their related archeological features, many of which were contemporary with 41BX2068 or slightly older or younger. Eight sites provided archeological features for comparative purposes (Table 4).

Table 4. Summary of historic sites investigated during the Alamodome and Henry B. Gonzalez Convention Center projects that were used for comparison to $41 \mathrm{BX2068}$

\begin{tabular}{|c|c|c|c|c|}
\hline Site Number & Dates & Feature Type & Description & Source \\
\hline $41 \mathrm{BX} 883$ & $1880-1890 \mathrm{~s}$ & privy vault & brick-lined vault; square & Wright 1997 \\
\hline $41 \mathrm{BX} 890$ & $1850-1870 \mathrm{~s}$ & privy vault & $\begin{array}{l}\text { cedar post-lined vault; } 63 \text { inches deep, } \\
\text { square }\end{array}$ & Wright 1997 \\
\hline $41 \mathrm{BX} 892$ & $1850-1860 \mathrm{~s}$ & privy vault & $\begin{array}{l}\text { wooden crate-lined privy vault; } 56 \\
\text { inches deep; square }\end{array}$ & Wright 1997 \\
\hline 41BX892 & $1850-1860 \mathrm{~s}$ & trash pit & $3 \mathrm{ft}$ diameter; 14 inches deep & Wright 1997 \\
\hline 41BX892 & post 1877 & privy vault & brick-lined vault; square & Wright 1997 \\
\hline 41BX892 & $1850-1870 \mathrm{~s}$ & trash pit & shallow trash scatter & Wright 1997 \\
\hline $41 \mathrm{BX} 896$ & $1850 \mathrm{~s}$ & $\begin{array}{l}\text { privy vault/ } \\
\text { trash pit }\end{array}$ & $\begin{array}{l}\text { earthen privy vault; } 60 \text { inches deep; } 3-4 \\
\mathrm{ft} \text { square at surface; } 2 \mathrm{ft} \text { square at base }\end{array}$ & Wright 1997 \\
\hline 41BX900 & $1860 \mathrm{~s}$ & privy vault & earthen privy vault; 32 inches deep & Wright 1997 \\
\hline $41 \mathrm{BX} 945$ & $1850-1890 \mathrm{~s}$ & privy vault & $\begin{array}{l}\text { limestone block privy vault; } \\
76 \text { inches deep }\end{array}$ & Wright 1997 \\
\hline 41BX1298 & post 1850 & privy vault & brick-lined privy vault & Tennis and Cox 1998 \\
\hline 41BX1298 & 1879-1901 & privy vault & wood-lined privy vault & Tennis and Cox 1998 \\
\hline 41BX1296 & post 1877 & privy vault & brick-lined privy vault & Tennis and Cox 1998 \\
\hline
\end{tabular}

The focus of this comparison was to assess whether the pit at 41BX2068 originally was dug to serve as something other than a trash receptacle, with the most obvious competing interpretation being that it was a privy pit later reused for refuse discard. No features substantially like the 41BX2068 pit were found in the literature, but based on the sites listed in Table 4, the privy pit interpretation seems highly unlikely. At those sites, all identified privies were deep (5-6 ft), often lined vaults. Earlier privies were square earthen vaults, some of which were lined with wooden crates, cedar posts, or cut limestone blocks for support. Later privy vaults remained square but became very formal constructions lined with brick. Upon arrival of the railroad to San Antonio in 1877, bricks became readily available, so this date is often used as the earliest date for brick-lined privy vaults in this area (Wright 1997:43). The majority of the privies recorded during the Alamodome Project were brick construction, therefore dating to 1877 or later.

Another characteristic common to privies in this area is that they were not densely packed with artifacts. In all cases, privies did contain fragments of lost or 
discarded artifacts, but not in the amounts observed at 41BX2068. Several even showed signs of being cleaned out on occasion.

The pit at 41BX2068 is distinctly different than the privy pits identified at the other sites. It is roughly round, basin shaped, unlined, and densely packed with artifacts. Additionally, no "night soils" were observed. Hence, it seems most likely that it was dug purposefully to hold trash.

\section{Archival Research}

The trash pit at 41BX2068 is in the backyard of a house at 541 Water Street that belonged to the Volpert family from 1856 until 1944. When the neighborhood was platted, probably in the 1850 s, Thomas Jefferson Devine (1820-1890) owned the land. He held a substantial amount of land in Bexar County by 1843 and continued to acquire and sell local property throughout his lifetime (Bexar County, Ad Valorem Tax Records; Bexar County, Deed Records). Born in Halifax, Nova Scotia, Devine launched his career in New York, moved to Tallahasse in 1835, and studied law in Mississippi and Kentucky. He first practiced in La Grange, Texas, where he and Helen Elder married in 1844. The couple moved to San Antonio that year. Devine was appointed city attorney and elected district judge in 1851. After he sold the property on Water Street, Devine served on the state's 1861 Secession Convention as a member of the Committee of Public Safety, and was appointed judge of the Confederate Western District of Texas. Having taken an oath of allegiance to Mexico at the end of the Civil War, Devine was twice indicted for treason, but pardoned (Find a Grave 2009; Russell 2015). Well before that, however, the city surveyor, G. Freisleben, platted Devine's San Antonio town lots (Bexar County, Deed Records).

On October 4, 1856, Devine sold Lot 5, Block 5 of City Block 890 to Marcellus Volpert $^{1}$ for $\$ 175$ (Figure 29) (Bexar County, Deed Record O2:140). The 46.65-ft front of the lot faced Water Street and extended northwesterly-169.8 ft on the southwest side and $170.5 \mathrm{ft}$ on the northeast side-to the Alamo Ditch (City of San Antonio 1964:97). Volpert (1835-1888) immigrated from the Hesse region of Germany, departing from Bremen on November 1, 1852, and arriving in Galveston on January 1, 1853, aboard the J. W. Buddecke (City of San Antonio 1888; Galveston Historical Foundation 1853; U.S. Department of the Interior, Census Office 1870, 1880). From 1858 until 1862, when he served as a private in Company B of the Texas Third Infantry, First Infantry, Luckett's Regiment, Volpert's property was valued at \$200 (Bexar County, Ad Valorem Tax Records; National Archives and Records Administration n.d.). Seeking volunteers at recruiting camps in Austin and San Antonio, Philip N. Luckett initially took 648 men to protect the Rio Grande in October 1862. They marched to the Gulf Coast, arriving in Galveston in July 1863. These troops were reassigned in April 1864 to the Red River campaign front lines (Cutrer 2015). Volpert returned to San Antonio, where he worked as a stonemason, and occupied his stone house at 541 Water Street (Bexar County, Ad Valorem Tax Records).

$\overline{1}$ Sometimes recorded as Volbert. 


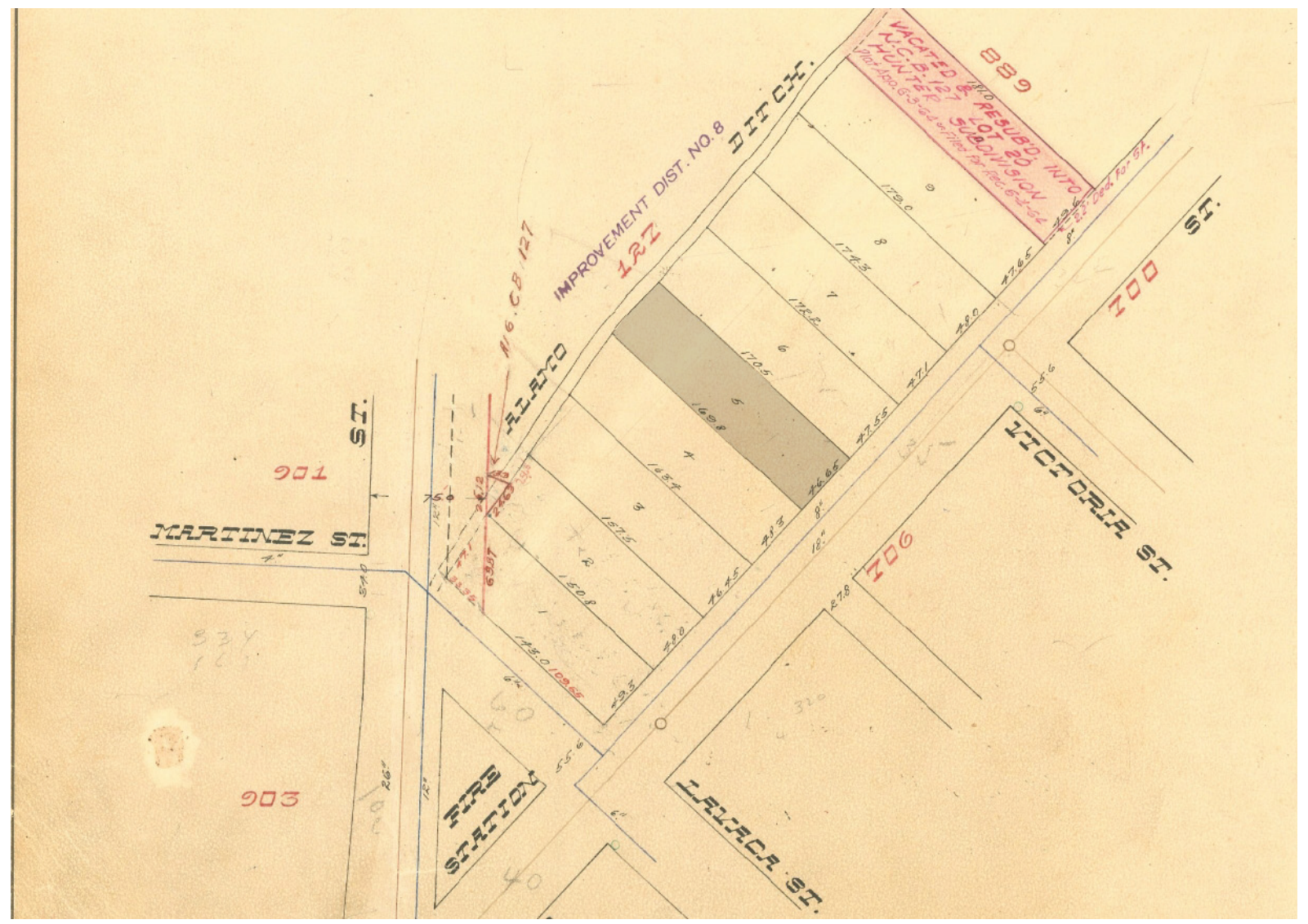

Figure 29. Map showing Lot 5, Block 5 of City Block 890 bounded by Water Street and the Alamo Ditch (City of San Antonio 1964).

Volpert and Cathryn Theis ${ }^{2}$ (1849-1879) married in February 1869. A native of the Nausau region of Germany, she had traveled to San Antonio with her parents in 1853. The family was farming in Bexar County in 1860, and she had three younger siblings at the time-Ferdinand, Margarita, and Paulina—all born in Texas (Bexar County, Marriage Record E:201; City of San Antonio 1879; U.S. Department of the Interior, Census Office 1860, 1870). In 1870, the Volperts had $\$ 800$ in real estate and $\$ 200$ in personal wealth (U.S. Department of the Interior, Census Office 1870). The value of their property grew slightly to $\$ 950$ in 1873 , remained static in 1874 and 1876 , and swelled to $\$ 1,500$ by 1877 (Bexar County, Ad Valorem Tax Records). During the 1870s, the couple had four children in quick succession: twin sons George M. (1871-1950) and Ferdinand (1871-1882), daughter Pauline (1874-1953), and son Charles John (1877-1928) (City of San Antonio 1882:48-49; Find a Grave 2008a, 2008b; San Antonio Express 1950; Texas Department of Health, Bureau of Vital Statistics 1950, 1953). Cathryn Theis Volpert died from heart disease on October 21, 1879, and was buried in the German Catholic cemetery (City of San Antonio 1879:104-105). As a widower, Volpert continued to reside in the 541 Water Street house, but the children attended Catholic schools and lived away from home, at least part of the time, after their mother's death (Bexar County, Ad Valorem Tax Records; Morrison \& Fourmy 1879; San Antonio Daily Light 1886:1; U.S.

\footnotetext{
2 Sometimes recorded as Tyce.
} 
Department of the Interior, Census Office 1880). In 1880, the two older boys lived on College Street with several of their teachers, servants, and other students; it is unclear where the two younger children lived that year (U.S. Department of the Interior, Census Office 1880). At age 12, Ferdinand Volpert succumbed to septicemia on December 5, 1882, and was buried in the German Catholic cemetery (City of San Antonio 1882:48-49). In 1885, Volpert was living at 439 Water Street (Morrison \& Fourmy 1885). Two years later, his youngest son, Charles, resided with his maternal grandmother, Anna Maria Bauldus Theis (1821-1901), the widow of George Theis (1816-1881) (Find a Grave 2008c; Morrison \& Fourmy 1888; U.S. Department of the Interior, Census Office 1860, 1880). By that time, both his older siblings were on their own; a merchant and manufacturer's agent employed Pauline, and George was working as a baker (Morrison \& Fourmy 1888). Volpert's property retained its value in the 1880 s, fluctuating between $\$ 1,200$, and $\$ 1,500$ (Bexar County, Ad Valorem Tax Records). On June 29, 1888, an hour after the onset of sunstroke, Volpert died at age 53, according to physician G. Graham Watts. He was also buried in the German Catholic Cemetery (City of San Antonio 1888:83-84).

Despite having their uncle, Lucas John Sueltenfuss, ${ }^{3}$ serving as a court-assigned guardian for the Volpert orphans-George was 15, Pauline was 14, and Charles was 11 when their father died - the estate struggled with payment of taxes on the family homestead. In July 1888 and May 1891, the Volpert orphans sought relief from payment (San Antonio Daily Light 1888:4, 1891:16). Nevertheless, the property retained its value in the 1890 s, hovering at $\$ 1,200$ and $\$ 1,250$ (Bexar County, Ad Valorem Tax Records). During this decade, the house may have been vacant, occasionally occupied by a family member, or housed tenants, such as A. G. Cotera in 1891 and Horace Hammant Hugman, a coachman for R. Winslow, in 1895 (Appler 1891, 1895). Meanwhile, siblings George and Pauline Volpert worked and lived elsewhere in San Antonio. In 1891, he was a clerk for C. H. Gemble; in 1892, he was a delivery boy for a company on Matagorda Street, and she was a servant for and resided with Mrs. Sallie Hamilton (Appler 1891, 1893). By 1896, the house had been divided into a duplex, and a long porch extended the breadth of the front southeast-facing façade. A small building was directly behind the house, and another was at the rear of the lot (Sanborn Fire Insurance Company 1896:28). In 1897, both George Volpert, who worked at a brick company, and Fred Arlitt, a harness maker for Frank and Company, occupied the house at 541 Water Street. That year, Charles Volpert was a laborer and lived elsewhere (Appler 1898). The following year, Charles Volpert and Emma M. (1879-1904) had married. The couple had moved to St. Louis by 1900 (U.S. Department of the Interior, Census Office 1900a). He lived out his life with his family in the St. Louis area (U.S. Department of Commerce, Bureau of the Census 1920a; U.S. Department of Commerce and Labor, Bureau of the Census 1910a). ${ }^{4}$

Probate matters continued for the Volpert heirs in the 1890s. Horace Hammant Hugman ${ }^{5}$ and Pauline Volpert married in Boerne on September 8, 1894

3 Sueltenfuss was an uncle to the children through his 1883 marriage to Theresa Theis, Cathryn Theis Volpert's youngest sister (Bexar County, Marriage Record H:179).

4 Emma M. Volpert died in 1904 as the result of a pregnancy (City of St. Louis, Health Department 1904). Charles Volpert married Wilhelmina "Minnie" Susanna Eckterhoff (1883-1927) in 1904 (Bexar County, Marriage Record S:546; Find A Grave 2008b). In 1910 the couple lived in St. Louis where he was a janitor for an office building (U.S. Department of Commerce and Labor, Bureau of the Census 1910a). They both died in Illinois (Find a Grave 2008b).

5 Horrace Hammant Hugman, brother to Robert Charles Harold Hugman, was uncle to Robert H. Harvey Hugman, who designed the River Walk. 
(Figure 30) (Bexar County, Marriage Record M:143). Consequently, the estate guardian was discharged in 1895 (San Antonio Daily Light 1895a:5, 1895b:8). However, in March 1897, the three Volpert heirs and Hugman filed suit against Sueltenfuss, as guardian for the minors, and two other men, for $\$ 3,000$ held in bond (San Antonio Daily Light 1897a:5, 1897b:5, 1897c:18). Near the end of the decade, Charles, the youngest of the Volpert heirs, sued his brother, sister, and brother-in-law to partition the land. H. B. Gray, who held a deed of trust on the property to secure a $\$ 160$ debt, was included in the suit. The court granted the partition, and the two older brothers separately conveyed their respective one-third interests to Horace Hammant Hugman between August 1898 and October 1899 for \$210; Pauline Volpert Hugman retained her one-third interest in the property (Bexar County, Deed Records 166:271, 167:380, 174:124, 176:435; San Antonio Daily Light 1898a:6, 1898b:6, 1899). The Hugmans occupied the property at 541 Water Street in 1897 and 1898 but had moved to 112 Peach Street by 1899 (Appler 1898).

Horace Hammant Hugman (1867-1907) was born in Tottenham, Middlesex, England. He immigrated to the United States through Baltimore in 1881 (British Isles Census 1881; National Archives and Records Administration 1881; San Antonio Light 1907:7). Horace and Pauline Volpert Hugman lived in the house they owned

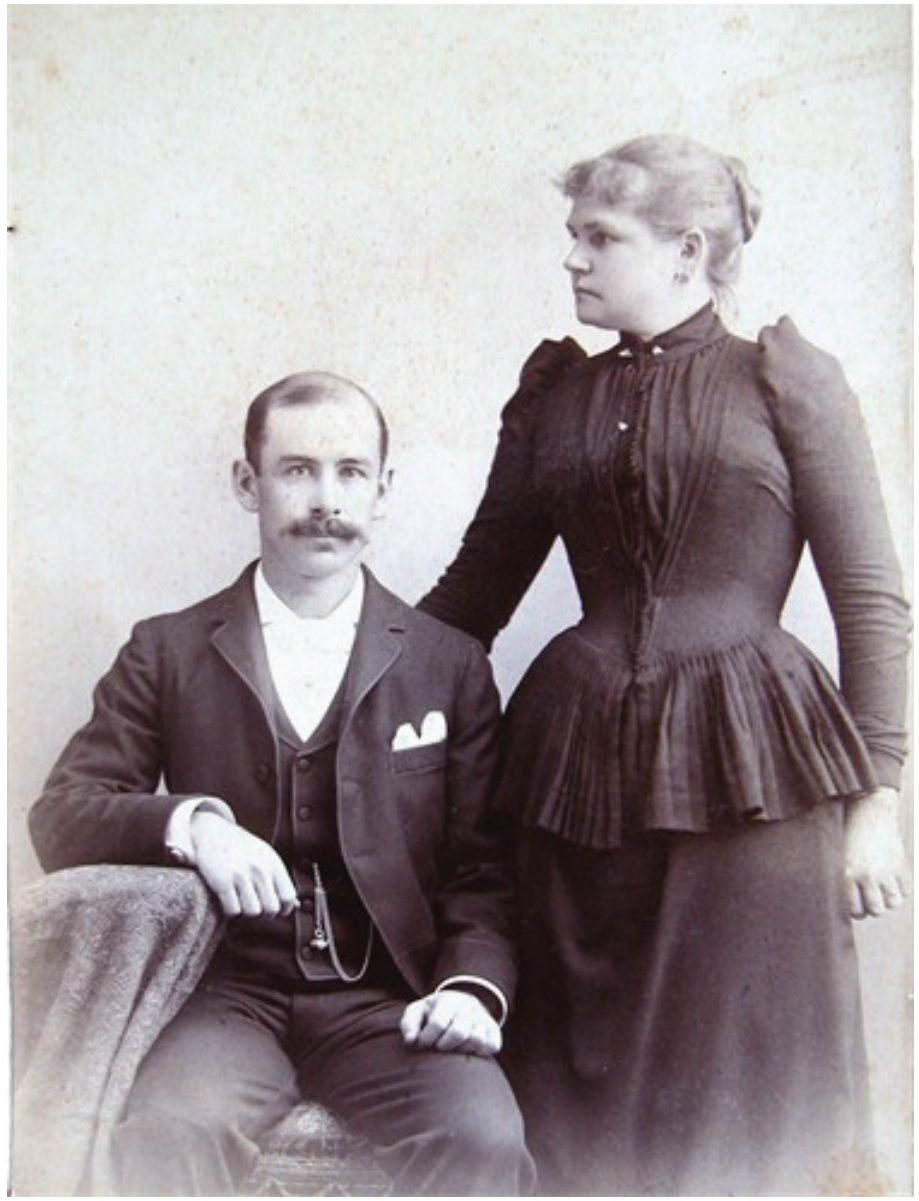

Figure 30. Horace Hammant and Pauline Volpert Hugman (Shaw Family Tree 2012). at 112 Peach Street in San Antonio in 1900 with two young children. He was employed as a hostler at the time (U.S. Department of the Interior, Census Office 1900b). When Hugman died in 1907, his widow inherited his interest in 541 Water Street (San Antonio Light 1907:7). In 1910, the widow lived with her five childrenGeorge, William, Florence, Alice, and Henry-on a farm she owned in Kendall County (U.S. Department of Commerce and Labor, Bureau of the Census 1910b). In 1919, she and William F. Cole (1867-1946) married (Bexar County, Deed Record 2065:328; Find a Grave 2008d; U.S. Department of Commerce, Bureau of the Census 1920a). A second marriage for both, their blended family lived on the Lower Balcones Road, where they owned a farm and ranch (U.S. Department of Commerce, Bureau of the Census 1920b). Ten years later, the couple remained on the property, but the children had moved on (U.S. Department of Commerce, Bureau of the Census 1930a). The Coles divorced in 1935, and she returned 
to her home at 112 Peach Street in San Antonio where she resided until her 1953 death (Bexar County, Deed Record 2065:328; John F. Worley Directory Company 1934-1935, 1948).

The oldest sibling, George Volpert and Dora Hagman (1880-1941), a native of Switzerland, married in June 1900 (Bexar County, Marriage Record P:390; San Antonio Daily Light 1900:4; Texas Department of Health, Bureau of Vital Statistics 1941). The couple divorced in 1907 (San Antonio Gazette 1907a:5, 1907b:1). Volpert, a baker, and several other Volpert heirs had received a sum of money from the sale of land in Bexar County in April 1907 (Bexar County, Deed Record 265:253). He traveled to Lockhart a few weeks before June 12, but apparently disappeared. Volpert was spotted in nearby Dale walking along the railroad with a bundle over his arm and was last seen headed to Plum Creek with a fishing pole. A cryptic note, with photographs of the couple and a wide black border, instructed his ex-wife to take his property because she would never see him again. This message and its presentation led to the assumption that Volpert was suicidal (San Antonio Gazette 1907a:5, 1907b:1). But he was back in San Antonio at his widowed sister's home at 112 Peach Street by February 1908, when he placed a newspaper advertisement to take in orders for silverware, transparent pocketknives, and earthenware (San Antonio Daily Light 1908:40). In 1920, Volpert was single and renting a farm on Ruby Street in the Hot Wells area, south of downtown San Antonio (U.S. Department of Commerce, Bureau of the Census 1920c). Ten years later, he was renting a farm on the old Corpus Christi Road as a widower ${ }^{6}$ (U.S. Department of Commerce, Bureau of the Census 1930b). Volpert had moved to Boerne by 1938, where he was a baker (San Antonio Express 1950:19). By 1940, he was single, no longer working, and renting a property in Boerne (U.S. Department of Commerce, Bureau of the Census 1940). He had a surviving son, George Jr., ${ }^{7}$ when he died in 1950 and was buried in the Boerne Cemetery (Find a Grave 2008a; San Antonio Express 1950:19; Texas Department of Health, Bureau of Vital Statistics 1950).

No members of the Volpert family occupied the dwelling at 541 Water Street in the twentieth century. Various tenants of the duplex included Max W. Fues, a clerk at Guenther Mills, in 1899 and 1900; Lamen W. Pytel and John E. Putnam, both employed by Fire Company \#3, in 1903 and 1904; N. B. Cozby, a printer, in 1905 and 1906; Alex Rossy, a cigar manufacturer, in 1907 and 1908; Emil, an upholsterer, and Mary Arandt in 1908; George, a well driller, and Jennie Antwright in 1909; August R., a butcher, and Agnes Townsend, Albert T. Townsend, a carpenter, and Albert, a carpenter, and Darthulia Robinson, all in 1910; Marcellino, a tailor, and Maria Medina from 1912 until 1918; Reyes C., a tailor, and Cleos Briones in 1913; John Leos, a tailor, in 1913 and 1914; Guadalupe Garansway, who worked at the Webster Company, in 1915; Gregory P. Garza, with Central Meat Market, John P.

$\overline{6}$ Both the 1920 census and his death certificate denote George M. Volpert as a widower, but no known record documents either a second marriage or the death of a spouse (Texas Department of Health, Bureau of Vital Statistics 1950; U.S. Department of Commerce, Bureau of the Census 1920c).

7 This is according to George M. Volpert's obituary, but no known record documents the life of a namesake. Volpert had a son, Alexander Murphy (1902-1968), with his first wife, and her second husband had adopted the boy by 1910 (FamilySearch 1951; Find a Grave 2011; U.S. Department of Commerce, Bureau of the Census 1930b; U.S. Department of Commerce and Labor, Bureau of the Census 1910c). 
Garza, a clerk at Halff Brothers, and Beatrice Uribe, a seamstress for a trunk company, in 1916; and F. Martinez, who worked at the Cream Dairy Company, and Mrs. Teresa Martinez in 1919 (Appler 1900 1902, 1904, 1906, 1908a, 1908b, 1909, 1910, 1912, 1913, 1914, 1915, 1916, 1917, 1918, 1919).

With so many tenant occupants, the house evolved in the early twentieth century. By 1904, the building was noted as a single-family dwelling despite the number of tenant occupants. The outbuilding closest to the house remained extant, but the one near the rear of the lot was no longer present (Sanborn Fire Insurance Company 1904:117). By 1912, two outbuildings were on the lot (Sanborn Fire Insurance Company 1912:238). In 1915, Pauline Volpert Hugman paid the Beebe Construction Company $\$ 194.70$ for curb and pavement improvements to the lot (Bexar County, Mechanics Lien 19:32). Street numbers changed after 1919, according to annual city directories and decennial census records, and it is unclear which pertained to the Volpert house. Even so, it is evident that the house was either vacant or tenant occupied between 1920 and 1944.

On July 25, 1944, Pauline Volpert Hugman Cole sold the property to Marcelino A. (1890-1978) and Maria (1890s-after 1978) Apolinar (Bexar County, Deed Record 2061:476; Find a Grave 2012; Texas Department of Health, Bureau of Vital Statistics 1978). This marked the end of an era for the Volpert family, who had continuously owned the land since 1856.

\begin{abstract}
Assessment
It is recommended that 41BX2068 be considered not eligible for listing in the National Register of Historic Places or designation as a State Antiquities Landmark. The feature did yield some useful information about material culture, consumer habits, and foodways in San Antonio during the latter half of the nineteenth century, but having been almost completely excavated, the feature contains no additional important information that would make it eligible for listing or designation.
\end{abstract}




\section{REFERENCES CITED}

Appler, Jules A., compiler

1891 General Directory of the City of San Antonio, 1891. Jules A. Appler, San Antonio. Electronic document, Ancestry.com, subscription database, accessed April 22, 2015.

1893 General Directory of the City of San Antonio, 1892-1893. Jules A. Appler, San Antonio. Electronic document, Ancestry.com, subscription database, accessed April 22, 2015.

1895 General Directory of the City of San Antonio, 1894-1895. Jules A. Appler, San Antonio. Electronic document, Ancestry.com, subscription database, accessed April 22, 2015.

1898 General Directory of the City of San Antonio, 1897-1898. Jules A. Appler, San Antonio. Electronic document, Ancestry.com, subscription database, accessed April 22, 2015.

1900 General Directory of the City of San Antonio, 1899-1900. Jules A. Appler, San Antonio. Electronic document, Ancestry.com, subscription database, accessed April 22, 2015.

1902 General Directory of the City of San Antonio, 1901-1902. Jules A. Appler, San Antonio. Electronic document, Ancestry.com, subscription database, accessed April 22, 2015.

1904 General Directory and Blue Book of the City of San Antonio, 1903-1904. Jules A. Appler, San Antonio. Electronic document, Ancestry.com, subscription database, accessed April 22, 2015.

1906 General Directory and Blue Book of the City of San Antonio, 1905-1906. Jules A. Appler, San Antonio. Electronic document, Ancestry.com, subscription database, accessed April 22, 2015.

1908a General Directory and Blue Book of the City of San Antonio, 1907-1908. Jules A. Appler, San Antonio. Electronic document, Ancestry.com, subscription database, accessed April 22, 2015.

1908b General Directory and Blue Book of the City of San Antonio, 1908. Jules A. Appler, San Antonio. Electronic document, Ancestry.com, subscription database, accessed April 22, 2015.

1909 General Directory and Blue Book of the City of San Antonio, 1909. Jules A. Appler, San Antonio. Electronic document, Ancestry.com, subscription database, accessed April 22, 2015.

1910 General Directory and Blue Book of the City of San Antonio, 1910. Jules A. Appler, San Antonio. Electronic document, Ancestry.com, subscription database, accessed April 22, 2015.

1912 General Directory and Blue Book of the City of San Antonio, 1912. Jules A. Appler, San Antonio. Electronic document, Ancestry.com, subscription database, accessed April 22, 2015. 
1913 General Directory and Blue Book of the City of San Antonio, 1913. Jules A. Appler, San Antonio. Electronic document, Ancestry.com, subscription database, accessed April 22, 2015 .

1914 General Directory and Blue Book of the City of San Antonio, 1914. Jules A. Appler, San Antonio. Electronic document, Ancestry.com, subscription database, accessed April 22, 2015.

1915 General Directory and Blue Book of the City of San Antonio, 1915. Jules A. Appler, San Antonio. Electronic document, Ancestry.com, subscription database, accessed April 22, 2015.

1916 General Directory and Blue Book of the City of San Antonio, 1916. Jules A. Appler, San Antonio. Electronic document, Ancestry.com, subscription database, accessed April 22, 2015.

1917 General Directory and Blue Book of the City of San Antonio, 1917. Jules A. Appler, San Antonio. Electronic document, Ancestry.com, subscription database, accessed April 22, 2015.

1918 General Directory and Blue Book of the City of San Antonio, 1918. Jules A. Appler, San Antonio. Electronic document, Ancestry.com, subscription database, accessed April 22, 2015.

1919 General Directory and Blue Book of the City of San Antonio, 1919. Jules A. Appler, San Antonio. Electronic document, Ancestry.com, subscription database, accessed April 22, 2015.

Bexar County

Ad Valorem Tax Records. Texas State Library and Archives Commission, Austin.

Deed Records. Bexar County Clerk, San Antonio. Electronic document, Bexar County Clerk database, accessed April 22, 2015.

Marriage Records. Bexar County Clerk, San Antonio. Electronic document, Bexar County Clerk database, accessed April 22, 2015.

Mechanic's Liens. Bexar County Clerk, San Antonio. Electronic document, Bexar County Clerk database, accessed April 22, 2015.

Boyd, Douglas K., Aaron R. Norment, Terri Myers, Maria Franklin, Nedra Lee, Leslie L. Bush, and Brian S. Schaffer

2015 The Ransom and Sarah Williams Farmstead: Post-Emancipation Transitions of an African American Family in Central Texas. Reports of Investigations No. 173. Prewitt and Associates, Inc., Austin.

British Isles Census

1881 Census Returns of England and Wales, 1881. Electronic document, Ancestry.com, subscription database, accessed April 22, 2015.

City of St. Louis, Health Department

1904 Certificate of Death, s.v. "Emma M. Volpert." Electronic document, Ancestry.com, subscription database, accessed April 22, 2015.

City of San Antonio

1879 Death Records, 1873-1884, s.v. “Catherine Volpert.” Electronic document, https:// webapps.sanantonio.gov/archivesearch/Viewer2.aspx?Id=\{0DCA0DBE-6CE5-487C9708-13FF464FE8B7\}\&DocTitle=Death\%20Records\%201873-1884\&PageNo=53\&Tot alPages=118\&MimeType=image/jpeg\&RelatedDocs=, accessed April 22, 2015. 
1882 Death Records, 1880-1885, s.v. "Ferdinan Volbert [sic].” Electronic document, https:// webapps.sanantonio.gov/archivesearch/Viewer2.aspx?Id=\{A90593B5-310E-4F7E-9F0697D5107B1794\}\&DocTitle=Death\%20Records\%201885-1890\%20\&PageNo=83\&TotalP ages=189\&MimeType=image/jpeg\&RelatedDocs=, accessed April 22, 2015.

1888 Death Records, 1885-1890, s.v. “Marcellus Volpert.” Electronic document, https:// webapps.sanantonio.gov/archivesearch/Viewer2.aspx?Id=\{A90593B5-310E-4F7E-9F0697D5107B1794\}\&DocTitle=Death\%20Records\%201885-1890\%20\&PageNo=83\&TotalP ages=189\&MimeType=image/jpeg\&RelatedDocs=, accessed April 22, 2015.

1964 New City Block Red Tax Maps, 800 through 899. Electronic document, https:// webapps.sanantonio.gov/archivesearch/Viewer2.aspx?Id=\{05AE8CA6-84FD-43A9996F-43BB8496AB61\}\&DocTitle=New\%20City\%20Block\%20Red\%20Tax\%20 Maps\%20800\%20through\%20899\&PageNo=97\&TotalPages=108\&MimeType=image/ jpeg\&RelatedDocs=, accessed April 22, 2015.

Cox, I. Waynne

2005 The Spanish Acequias of San Antonio. Maverick Publishing Company, San Antonio, Texas.

Cutrer, Thomas

2015 The Handbook of Texas Online, s.v. "Philip Noland Luckett.” Electronic document, https:// tshaonline.org/handbook/online/articles/flu05, accessed April 22, 2015.

Dase, Amy E.

2013 Historical Resources Studies: Proposed HemisFair Park Area Redevelopment, San Antonio, Bexar County, Texas. Letter Report No. 867. Prewitt and Associates, Inc., Austin.

Dupont, William A., Saadet Beeson, Sedef Doganer, Adriana Muñoz, and Laura Carrera

2011 Inventory, Assessment, and Evaluation of Historic Resources in HemisFair Park. UTSA Center for Cultural Sustainability, The University of Texas at San Antonio.

FamilySearch

1951 Texas, Births and Christenings, s.v. "Alexander [Volpert] Murphy.” Electronic document, https://familysearch.org/ark:/61903/1:1:F6PN-MVY, accessed April 22, 2015.

Feit, Rachel

2009 Archeological Survey in San Antonio’s Hemisfair Park, Bexar County, Texas. Ecological Communications Corporation, Austin.

Fields, Ross C., and Jennifer K. McWilliams

2012 Archeological File Search and Literature Review, Proposed HemisFair Park Redevelopment, San Antonio, Texas. Prewitt and Associates, Inc., Austin.

Find a Grave

2008a Find a Grave, s.v. "George M. Volpert." Electronic document, http://findagrave.com/cgibin/fg.cgi?page=gr\&GRid=32291582, accessed April 22, 2015.

2008b Find a Grave, s.v. "Charles John Volpert." Electronic document, http://findagrave.com/ cgi-bin/fg.cgi?page=gr\&GRid=32266201, accessed April 22, 2015.

2008c Find a Grave, s.v. "Anna Maria Bauldus Theis.” Electronic document, http://findagrave. com/cgi-bin/fg.cgi?page=gr\&GRid=26664971, accessed April 22, 2015.

2008d Find a Grave, s.v. "William F. Cole." Electronic document, http://findagrave.com/cgi-bin/ fg.cgi?page=gr\&GRid=32446621, accessed April 22, 2015.

2009 Find a Grave, s.v. “Thomas Jefferson Devine.” Electronic document, http://findagrave. com/cgi-bin/fg.cgi?page=gr\&GRid=42493954, accessed April 22, 2015. 
2011 Find a Grave, s.v. "Alexander Murphy." Electronic document, http://findagrave.com/cgibin/fg.cgi?page=gr\&GRid=76317754, accessed April 22, 2015.

2012 Find a Grave, s.v. "Marcelino A. Apolinar.” Electronic document, http://findagrave.com/ cgi-bin/fg.cgi?page=gr\&GRid=86960652, accessed April 22, 2015.

Foster, Eugene R. Jr., Ty Summerville, and Thomas Brown

2006 The Texas Historic Overlay: A Geographic Information System of Historic Map Images for Planning Transportation Projects in Texas. Document No. 060206. PBS\&J, Austin.

Fox, Anne A.

1985 Testing for the Location of the Alamo Acequia (41BX8) at HemisFair Plaza, San Antonio, Texas. Archaeological Survey Report No. 142. Center for Archaeological Research, The University of Texas at San Antonio.

Fox, Anne A., and I. Waynne Cox

1990 Archaeological Excavations at the Alamo Acequia, Southwest HemisFair Plaza, San Antonio, Bexar County, Texas. Archaeological Survey Report No. 192. Center for Archaeological Research, The University of Texas at San Antonio.

Galveston Historical Foundation

1853 Galveston Immigration Database, s.v. "Marcellus Volbert [sic]." Electronic document, http://ghf.destinationnext.com/immigration/SearchDetails.aspx?ID=17438, accessed April 22, 2015.

Gast, August, and Co.

1883 "Morrison and Fourmy's Revised Map of City of San Antonio Texas." In The Texas Historic Overlay: A Geographic Information System of Historic Map Images for Planning Transportation Projects in Texas, by Eugene R. Foster Jr., Ty Summerville, and Thomas Brown, 2006. Document No. 060206. PBS\&J, Austin.

Godden, Geoffrey A.

1991 Encyclopedia of British Pottery and Porcelain Marks. Barrie \& Jenkins, London.

Greer, Georgeanna H.

1981 American Stonewares, The Art and Craft of Utilitarian Potters. Schiffer Publishing Limited, Exton, Pennsylvania.

HemisFair Park Area Redevelopment Corporation

2012 HemisFair Park Area Master Plan. Prepared by Johnson Fain, Olin, HR\&A Advisors, Poteet Architects, UTSA/Dupont, Pape-Dawson, Arup, and R. J. Rivera.

Johnson, Edgar D, and I. Waynne Cox

1995 An Archival and Archaeological Study for the Relocation of Three Historic Homes in HemisFair Park, San Antonio, Texas. Archaeological Survey Report No. 241. Center for Archaeological Research, The University of Texas at San Antonio.

Koch, Augustus

1873 Bird's Eye View of the City of San Antonio, Bexar County, Texas, 1873. Texas Bird's-Eye Views. Amon Carter Museum, Fort Worth, Texas. Available at http://www.birdseyeviews. org/index.php.

1886 Bird's Eye View of San Antonio, Bexar County, Texas, 1886, Looking East. Texas Bird's-Eye Views. Amon Carter Museum, Fort Worth, Texas. Available at http://www.birdseyeviews. org/index.php.

Kovel, Ralph, and Terry Kovel

1986 Kovels' New Dictionary of Marks: Pottery \& Porcelain 1850 to the Present. Crown Publishers, Inc., New York. 
Lindsey, Bill

2015 Bottle Dating: Machine-Made Bottles. In Historic Glass Bottle Identification \& Information Website. Electronic document, http://www.sha.org/bottle/machinemadedating. htm\#Machinemade\%20characteristics, accessed January 30, 2015.

Morrison \& Fourmy, compilers

1879 General Directory of the City of San Antonio, 1879. Jennings Brothers, Marshall, Texas.

1885 General Directory of the City of San Antonio, 1885. Morrison \& Fourmy, Galveston, Texas.

1888 General Directory of the City of San Antonio, 1887-1888. Morrison \& Fourmy, Galveston, Texas.

National Archives and Records Administration

n.d. Compiled Service Records of Confederate Soldiers Who Served in Organizations from the State of Texas. Third Infantry (First Infantry, Luckett's Regiment). Electronic document, Ancestry.com, subscription database, accessed April 22, 2015.

1881 Baltimore, Passenger Lists, 1827-1964. Electronic document, Ancestry.com, subscription database, accessed April 22, 2015.

Olsen, J. J., and Son

1889 "City of San Antonio." In The Texas Historic Overlay: A Geographic Information System of Historic Map Images for Planning Transportation Projects in Texas, by Eugene R. Foster Jr., Ty Summerville, and Thomas Brown, 2006. Document No. 060206. PBS\&J, Austin.

Ramsay, John

1939 American Potters and Pottery. Hale, Cushman, and Flint, New York.

Russell, Yancey L.

2015 The Handbook of Texas Online, s.v. "Thomas Jefferson Devine." Electronic document, https://tshaonline.org/handbook/online/articles/fde50, accessed April 22, 2015.

San Antonio Daily Light

1886 "Catholic Schools." San Antonio Daily Light, July 1, 1886. Electronic document, Newspaper Archive, subscription database, accessed April 22, 2015.

1888 "City Wiseacres." San Antonio Daily Light, July 17, 1888. Electronic document, Newspaper Archive, subscription database, accessed April 22, 2015.

1891 "City Council." San Antonio Daily Light, May 5, 1891. Electronic document, Newspaper Archive, subscription database, accessed April 22, 2015.

1895a "Court News: Probate Matters." San Antonio Daily Light, January 25, 1895. Electronic document, Newspaper Archive, subscription database, accessed April 22, 2015.

1895b “Court News: Probate Matters." San Antonio Daily Light, March 27, 1895. Electronic document, Newspaper Archive, subscription database, accessed April 22, 2015.

1897a “New Suits Filed." San Antonio Daily Light, March 24, 1897. Electronic document, Newspaper Archive, subscription database, accessed April 22, 2015.

1897b “Probate Court." San Antonio Daily Light, April 14, 1897. Electronic document, Newspaper Archive, subscription database, accessed April 22, 2015.

1897c “City Tax List.” San Antonio Daily Light, October 16, 1897. Electronic document, Newspaper Archive, subscription database, accessed April 22, 2015. 
1898a "Suit for Partition." San Antonio Daily Light, September 12, 1898. Electronic document, Newspaper Archive, subscription database, accessed April 22, 2015.

1898b "Real Estate Transfers." San Antonio Daily Light, September 30, 1898. Electronic document, Newspaper Archive, subscription database, accessed April 22, 2015.

1899 "Real Estate Transfers." San Antonio Daily Light, October 25, 1899. Electronic document, Newspaper Archive, subscription database, accessed April 22, 2015.

1900 "Light Rays." San Antonio Daily Light, June 26, 1900. Electronic document, Newspaper Archive, subscription database, accessed April 22, 2015.

1908 "Wanted-Miscellaneous." San Antonio Daily Light, February 2, 1908. Electronic document, Newspaper Archive, subscription database, accessed April 22, 2015.

San Antonio Express

1950 “G. M. Volpert.” San Antonio Express, February 23, 1950. Electronic document, Newspaper Archive, subscription database, accessed April 22, 2015.

San Antonio Gazette

1907a "Mysterious Disappearance." San Antonio Gazette, June 12, 1907. Electronic document, Newspaper Archive, subscription database, accessed April 22, 2015.

1907b “Missing Man Sent Divorce Wife Farewell." San Antonio Gazette, June 18, 1907. Electronic document, Newspaper Archive, subscription database, accessed April 22, 2015.

San Antonio Light

1907 "Horace Hugman." San Antonio Light, December 31, 1907. Electronic document, Newspaper Archive, subscription database, accessed April 22, 2015.

Sanborn Fire Insurance Company

1892 San Antonio, Bexar County, Texas. Sanborn Fire Insurance Company, New York. Electronic document, Digital Sanborn Maps, 1867-1970.

1896 San Antonio, Bexar County, Texas. Sanborn Fire Insurance Company, New York. Electronic document, Digital Sanborn Maps, 1867-1970.

1904 San Antonio, Bexar County, Texas. Sanborn Fire Insurance Company, New York. Electronic document, Digital Sanborn Maps, 1867-1970.

1912 San Antonio, Bexar County, Texas. Sanborn Fire Insurance Company, New York. Electronic document, Digital Sanborn Maps, 1867-1970.

Schuetz, Mardith K.

1970 Excavation of a Section of the Acequia Madre in Bexar County, Texas, and Archeological Investigations at Mission San José in April 1968. Archeological Report Number 19. Texas Historical Survey Committee, Austin.

Shaw Family Tree

2012 Horace Hemmant Hugman and Pauline Volpert [photograph]. Electronic document, Ancestry.com, subscription database, accessed April 22, 2015.

Sprague, Roderick

2002 China or Prosser Button Identification and Dating. Historical Archaeology 36(2):111-127.

Taylor, F. B., R. B. Hailey, and D. L. Richmond

1991 Soil Survey of Bexar County, Texas. United States Department of Agriculture, Soil Conservation Service, in cooperation with the Texas Agriculture Experiment Station. 
Tennis, Cynthia L., and I. Waynne Cox

1998 Archaeological Monitoring for the City of San Antonio: Henry B. Gonzalez Convention Center Expansion. Archaeological Survey Report No. 284. Center for Archaeological Research, The University of Texas at San Antonio.

Texas Department of Health, Bureau of Vital Statistics

1941 Certificate of Death, s.v. “Dora [Hagman Volpert] Murphy.” Electronic document, Ancestry. com, subscription database, accessed April 22, 2015.

1950 Certificate of Death, s.v. "George M. Volpert.” Electronic document, Ancestry.com, subscription database, accessed April 22, 2015.

1953 Certificate of Death, s.v. "Pauline Hugman Cole." Electronic document, Ancestry.com, subscription database, accessed April 22, 2015.

1978 Certificate of Death, s.v. "Marcelino A. Apolinar." Electronic document, Ancestry.com, subscription database, accessed April 22, 2015.

Tobin International, Ltd.

1939 Bexar County, aerial photograph. Available from P2 Energy Solutions, San Antonio.

U.S. Department of Commerce, Bureau of the Census

1920a St. Louis County, Missouri, manuscript population schedule. Electronic document, HeritageQuest Online, subscription service, accessed April 22, 2015.

1920b Kendall County, Texas, manuscript population schedule. Electronic document, HeritageQuest Online, subscription service, accessed April 22, 2015.

1920c Bexar County, Texas, manuscript population schedule. Electronic document, HeritageQuest Online, subscription service, accessed April 22, 2015.

1930a Kendall County, Texas, manuscript population schedule. Electronic document, HeritageQuest Online, subscription service, accessed April 22, 2015.

1930b Bexar County, Texas, manuscript population schedule. Electronic document, HeritageQuest Online, subscription service, accessed April 22, 2015.

1940 Bexar County, Texas, manuscript population schedule. Electronic document, HeritageQuest Online, subscription service, accessed April 22, 2015.

U.S. Department of Commerce and Labor, Bureau of the Census

1910a St. Louis County, Missouri, manuscript population schedule. Electronic document, HeritageQuest Online, subscription service, accessed April 22, 2015.

1910b Kendall County, Texas, manuscript population schedule. Electronic document, HeritageQuest Online, subscription service, accessed April 22, 2015.

1910c Bexar County, Texas, manuscript population schedule. Electronic document, HeritageQuest Online, subscription service, accessed April 22, 2015.

U.S. Department of the Interior, Census Office

1860 Bexar County, Texas, manuscript population schedule. Electronic document, HeritageQuest Online, subscription service, accessed April 22, 2015.

1870 Bexar County, Texas, manuscript population schedule. Electronic document, HeritageQuest Online, subscription service, accessed April 22, 2015.

1880 Bexar County, Texas, manuscript population schedule. Electronic document, HeritageQuest Online, subscription service, accessed April 22, 2015. 
1900a St. Louis County, Missouri, manuscript population schedule. Electronic document, HeritageQuest Online, subscription service, accessed April 22, 2015.

1900b Bexar County, Texas, manuscript population schedule. Electronic document, HeritageQuest Online, subscription service, accessed April 22, 2015.

Wells, Tom

1998 Nail Chronology: The Use of Technologically Derived Features. Historical Archaeology 32(2):78-99.

Worley, John F., Directory Company

1934-1935 Worley's San Antonio (Texas), City Directory 1934-1935. John F. Worley Directory Company, Dallas.

1948 Worley's San Antonio (Bexar County, Texas), City Directory 1948. John F. Worley Directory Company, Dallas.

Wright, G. Kenneth

1997 Archaeology at the Alamodome: Investigations of a San Antonio Neighborhood in Transition, Volume II, Excavations and Artifact Distribution Analysis, edited by Anne A. Fox, Marcie Renner, and Robert J. Hard. Archaeological Survey Report, No. 237. Center for Archaeological Research, The University of Texas at San Antonio. 


\title{
APPENDIX A: Analysis of Vertebrate Faunal Remains from 41BX2068
}

\author{
Barbara A. Meissner \\ Raba Kistner Environmental, Inc. \\ San Antonio, Texas
}





\section{INTRODUCTION}

On behalf of Raba Kistner Environmental, Inc. (RKEI), the author conducted systematic faunal analysis of a collection of remains recovered by Prewitt and Associates, Inc., from 41BX2068. The faunal assemblage was received by RKEI in April 2015 and was transferred to the author in early May 2015. The assemblage consisted of a total of 919 bones, weighing 13,234.29 g. The faunal materials had been washed, dried, and bagged in a single provenience representing the entire trash pit.

\section{METHODS}

Taxonomy is not a static framework in biology. Research in the placement (and thus the naming) of species in the taxonomic hierarchy is ongoing in many cases. The authority used for taxonomic nomenclature for this report is the Integrated Taxonomic Information System (ITIS), an international collaboration of taxonomists available online (ITIS 2015).

Each bone was identified to the most specific taxon possible using several reference texts (Balkwill and Cumba 1992; Cohen and Serjeantson 1996; Gilbert 1990; Gilbert et al. 1981; Hillson 1955; Olsen 1960, 1964, 1968). Identification was conservative, e. g., bone that was cow-sized was not identified as cattle (Bos taurus) unless it could be positively differentiated from bison (Bison bison) and horse (Equus caballos). When identification to a taxonomic level was not certain, the bone was assigned to the next highest taxonomic level. Thus, bone that was clearly bovid but that could not be differentiated between cattle and bison was listed as Bovinae. Bone that could only be identified to class, such as mammal or bird, was separated by size when possible. Bones that had clearly been broken during excavation and could be matched were counted as a single bone. When unsealed epiphyses were present and could be mended to the diaphysis, they were counted as one bone.

All bone was weighed, and evidence of heat alteration (charred or calcined) and cutting with saws was noted. For bone identifiable to at least the order taxonomic level, additional data recovered included element and portion of element, side, evidence of juvenile characteristics, and butcher marks. In addition, when feasible, the thickness of the "cut" was measured.

Data recovered from the analysis was entered into a spreadsheet. Bone was bagged by taxa in 4-mil-thick polyethylene bags. Acid-free paper tags listing site number and number of bags for each taxon, as well as count and weight for each bag, were placed inside another bag to prevent contact with the faunal material.

Three measures of relative abundance were used to estimate the importance of all species to the meat diet as represented by this collection: Number of Identified Specimens (NISP), Minimum Number of Individuals (MNI), and bone weight. NISP is the count of specimens identified to at least the genus taxonomic level. Its use as a measure of relative abundance is problematic (Grayson 1984:20-24; Reitz and Wing 1999:191-192). NISP cannot differentiate between fragments from a single individual and fragments from many individuals. Reliance on NISP alone will distort the estimate of relative abundance in favor of small animals (Grayson 1984) as 
well as animals with parts that are more readily identifiable in fragmentary form (Lyman and O'Brien 1987).

Minimum Number of Individuals (MNI) is a derived measure commonly used to get around some of the biases of NISP. MNI is the minimum number of animals of each species or genera that must have been present to account for the elements identified for that species. This is determined by dividing paired elements (or portions of elements such as proximal or distal ends) into right and left side, and then finding the most abundant such element (Lyman 1994:38-39). Estimated age of the animals (at least immature vs. mature) is often considered in the analysis. MNI can, if properly calculated, be a useful counterpoint to NISP. However, MNI also has problems as a measure of relative abundance. Grayson (1984: 29-49) has pointed out that MNI is extremely sensitive to the way in which the analyst aggregates the data. MNI counts can be made to vary a great deal by changing how the analyst groups the data, for instance, by unit/level, by complete unit, by natural level, by the entire site, etc. (see Grayson 1984:34-49). The more inclusive the grouping, the more the data are reduced. In this case, the entire collection is from a single provenience and is estimated to have been deposited over a relatively short duration, thus the MNI estimate avoids many of the problems described by Grayson (1984).

Bone weight can also, with caution, be used as a measure of relative abundance. In general, larger bones carry more meat, but the relationship is not linear (Reitz and Wing 1999:222-231), varies among different taxa, and varies considerably from one part of the animal to another (e.g., lower legs of artiodactyls are dense, heavy bones but carry relatively little meat compared to other bones of the body). Using bone weight as an assessment of dietary importance also is limited by the necessary assumption that all taphonomic factors that affect bone weight (such as leaching, mineralization, or encrustation) have affected all bones in the collection uniformly. In this collection, the bones were probably deposited within a relatively short period and are in excellent condition, showing only slight weathering on a few bones, with none showing any encrustation. Bone weight tends to emphasize the importance of larger, heavier animals, and due to this, counterbalances the tendency of NISP to emphasize smaller animals.

Each analytic method discussed above is subject to a number of biases (Reitz and Wing 1999:200). Used together, however, NISP, MNI, and bone weight can provide a better picture of the relative importance of each species to the diet than any can provide when used alone.

\section{RESULTS}

A total of 80 bones, 8.7 percent of the total collection, representing 7 taxa were identified to at least the genus taxonomic level (Table A.1). Cattle (Bos taurus) was the most common identified species, with 40 bones, and pig (Sus scrofa) the next most common with 31 bones. All other identified genera were limited to 3 or fewer bones.

Although bone that could not be differentiated between cow and bison is listed as Bovinae in Table A.1, the fact that bison had been extirpated from the San 
Table A.1. List of vertebrate faunal taxa from 41BX2068

\begin{tabular}{|c|c|c|c|c|}
\hline Taxon & Common Name & Count & Weight (g) & MNI \\
\hline \multicolumn{5}{|c|}{ Mammalia: Family Artiodactyla } \\
\hline Bos taurus & Cow & 40 & $3,378.81$ & 5 \\
\hline Bovinae & Cow or bison & 230 & $6,787.65$ & 3 \\
\hline Capra hircus & Domestic goat & 3 & 71.59 & 2 \\
\hline Odocoileus virginianus & White-tailed deer & 1 & 16.18 & 1 \\
\hline Ovis aries & Domestic sheep & 2 & 14.20 & 1 \\
\hline Sus scrofa & Domestic pig & 31 & 747.24 & 2 \\
\hline Unidentified & Deer, sheep, goat & 25 & 336.32 & \\
\hline Total & & 332 & $11,351.99$ & \\
\hline \multicolumn{5}{|c|}{ Mammalia: Family Equidae } \\
\hline Equus sp. & Horses and donkeys & 2 & 67.05 & 1 \\
\hline \multicolumn{5}{|c|}{ Mammalia: Unidentified } \\
\hline Small & Rabbit-sized & 1 & 0.28 & \\
\hline Large & Goat/sheep-sized & 222 & 147.46 & \\
\hline Very large & Cow-sized & 255 & $1,639.99$ & \\
\hline Undetermined & & 103 & 8.69 & \\
\hline Total & & 581 & $1,796.42$ & \\
\hline Total Mammalia & & 915 & $13,215.46$ & \\
\hline \multicolumn{5}{|c|}{ Aves: Family Phasianidae } \\
\hline Meleagris gallopavo & Turkey & 1 & 13.53 & 1 \\
\hline \multicolumn{5}{|l|}{ Aves: Unidentified } \\
\hline Large & Chicken-sized & 1 & 1.11 & \\
\hline Very large & Turkey-sized & 2 & 4.19 & \\
\hline Total Aves & & 4 & 18.83 & \\
\hline Grand Total & & 919 & $13,234.29$ & \\
\hline
\end{tabular}

Antonio area by about 1840 (Weniger 1997:38) indicates that the Bovinae can be grouped with Bos taurus with a reasonable degree of comfort. This brings the total bovid count to 270 and increases the MNI to 8 . It should be noted that the MNI for pig is low probably because many of the identifiable pig bones, in particular the metapodials and phalanges, are not readily separated into left and right sides.

\section{BONE CONDITION}

The bones in this collection are in unusually good condition, with only a few showing slight weathering due to exposure, and none showing the chemical weathering caused by microbial activity that is common in open sites in south Texas. The deposition of the bones into a pit and the apparent rapid filling of the feature protected them from weathering and differential preservation. The absence of rodent chewing also indicates that the pit was filled fairly rapidly.

\section{EVIDENCE OF BURNING}

Only 118 bones (12.8 percent) in this collection were charred. Of these, 11 had only limited areas of charring, suggesting they were charred during cooking, possibly over an open fire. No bones in this collection were calcined. The more heavily 
charred bones suggest that bones were occasionally tossed into a fire for disposal and that the ashes of the fire were tossed into the trash pit.

\section{BUTCHER MARKS}

Four types of butcher marks were noted during data collection: (1) cut, a thin superficial cut from a knife $(\mathrm{n}=7)$; (2) chop, a deep cut from a large knife or hatchet ( $\mathrm{n}=110)$; (3) impact scar, evidence of small area blunt force impact $(\mathrm{n}=48)$; and (4) hand saw cut, with uneven striations and differing angles ( $\mathrm{n}=192)$. In addition, although usually not considered a butcher's mark per se, bullet holes were noted (see below). Many bones had more than one type of mark. No bones had the characteristic marks of a machine-powered saw. Machine saws were in use at least by larger operations in California by the 1920s, but small rural butchers were still using hacksaws to butcher meat (Gust 1983:344). While it is not known when power equipment became the norm in butchering in San Antonio, the presence of both hand-sawed and machine-sawed bones in a privy that was filled between roughly 1905 and 1920 (Brown and De La O 1997:267) suggests that at least some butchers in San Antonio were using power equipment within the first few decades of the twentieth century.

\section{DISCUSSION}

\section{Intersite Comparisons}

The richness of this collection is very limited, with only seven different genera. All of the taxa identified to genus are domestic animals, with the possible exception of turkey. All the taxa are commonly used food animals, with the exception of horse or donkey. Comparison of this collection to those from two sites dating to roughly the same period that were excavated prior to the building of the Alamodome in San Antonio shows that the dump at 41BX2068 is very different (Table A.2).

The Webb site (41BX897) was first occupied in 1865, and various members of the family continued to own the lot for the next hundred years (Meissner and Hunziker 1997:301). The trash dump was large, although relatively shallow, and was found to extend well into the lot to the south in one continuous unit. Historical records and the analysis of artifacts from the trash dump suggested that the dump was in use from the time the Webbs purchased the property until roughly the late 1880s (Tennis 1997:24). The lot to the south was sold in 1883, at which time a house was built there, and presumably the Webbs could no longer use the southern part of the dump. While the vast majority of the artifacts recovered were clearly mid to late nineteenth century, there were a few that were likely early twentieth century, including a few machine saw cut bones (Meissner and Hunziker 1997:323). The site to the south of the Webb's, the King/Klar site (41BX883) included a brick-lined privy constructed about 1890 (Brown and De La O 1997:261). Since the privy was filled with household trash after the house was attached to the new city sewage system about 1905, the artifacts and bones from this privy represent the early twentieth century. 
Table A.2. Comparison of faunal assemblage from 41BX2068 with those from roughly contemporaneous sites $41 \mathrm{BX897}$ and $41 \mathrm{BX883}$

\begin{tabular}{|c|c|c|c|}
\hline Characteristic & 41BX2068 & 41BX897 & 41BX883 \\
\hline Bone count & 919 & 3,274 & 1,895 \\
\hline No. of genera identified & 7 & 15 & 14 \\
\hline Percent identified to genus & 36.5 & 38.6 & 31.1 \\
\hline Percent unidentified mammals & 63.2 & 58.3 & 65.2 \\
\hline Percent juveniles & 31.3 & 19.6 & 17.4 \\
\hline \multicolumn{4}{|l|}{ Selected Genera } \\
\hline Bovids & $253(86.9 \%)$ & $921(72.4 \%)$ & $250(40.7 \%)$ \\
\hline Sheep/goat & $5(1.7 \%)$ & $96(7.5 \%)$ & $28(4.6 \%)$ \\
\hline Pig & $31(10.7 \%)$ & $179(14.1 \%)$ & $134(21.8 \%)$ \\
\hline Chicken & $0(0 \%)$ & $26(2.0 \%)$ & $37(6.0 \%)$ \\
\hline Commonly hunted animals & $1(0.3 \%)$ & $16(1.3 \%)$ & $76(12.4 \%)$ \\
\hline Birds & $1(0.3 \%)$ & $12(0.9 \%)$ & $49(8.0 \%)$ \\
\hline Fish & $0(0 \%)$ & $20(1.6 \%)$ & $39(6.3 \%)$ \\
\hline Other animals & $0(0 \%)$ & $2(0.2 \%)$ & $2(0.3 \%)$ \\
\hline Total & 291 & 1,272 & 615 \\
\hline \multicolumn{4}{|l|}{ Butcher Marks (selected) } \\
\hline Chop & $110(36.4 \%)$ & $388(44.7 \%)$ & $45(34.1 \%)$ \\
\hline Hand saw cut & $192(63.6 \%)$ & $453(52.2 \%)$ & $53(40.2 \%)$ \\
\hline Machine saw cut & $0(0 \%)$ & $27(3.1 \%)$ & $34(25.8 \%)$ \\
\hline Total & 302 & 868 & 132 \\
\hline
\end{tabular}

Table A.2 compares various attributes of the three collections. Note that 41BX2068 has far fewer bones than the much larger trash dump at the Webb site and the privy at the King/Klar site. Both the bones identified to genus and the count of bones that can only be identified as mammal are proxy estimates of the degree of fragmentation of the bones. All three collections are roughly similar in these estimates and show that the bones were in relatively the same condition, although the bones from the Webb dump were somewhat more fragmented, partially as a result of the way the dump was excavated (Wright 1997:88). The percentage of bones that had evidence of immaturity in the 41BX2068 collection was almost twice that of other two.

As noted, the collection from 41BX2068 has an unusually small number of identified genera. The difference among the three collections is largely in the wide range of wild animals present in the Webb trash dump and the King/Klar privy, especially in the latter, where there were large numbers of both jackrabbits (Lepus californicus) and cottontail rabbits (Sylvilagus sp.) as well as large numbers of ducks, geese, and turkey (Meissner and Hunziker 1997:321, 326).

Table A.2 also shows categories of animals in the three collections. All bone identified as bovid was grouped together, under the assumption that Bison (Bison bison) had been extirpated from the area around San Antonio by the time all three sites were inhabited (Weniger 1997:38). Goat and sheep bones were grouped because they were grouped by Meissner and Hunziker (1997) if they could be differentiated from deer but not from each other. The category "Commonly hunted animals" includes 
deer (Odocoileus virginianus) jackrabbit, cottontail rabbit, and javelina (Pecari tajacu). The "Birds" category includes ducks (Anatinae), geese (Anserinae), and turkeys (Meleagris gallopavo), all of which may or may not have been domesticated at the time they were killed. All fish bones, whether identified to genus or not, was included in "Fish" category.

In all three collections, bovids are most common, with pig the second most abundant. The privy at the King/Klar site (41BX883) is the odd man out, with far more hunted mammals and birds that may have been hunted than either of the other collections. During the time that the privy was being filled, the property was owned by a butcher (Brown and De La O 1997:263), and it is possible that he routinely included hunted animals in his shop, although the large numbers of jackrabbits are a puzzle since these animals produce notoriously tough meat (Weniger 1997:156). Site 41BX2068 is unique in the near-absence of anything but domestic animals, with only single deer and turkey bones. There were, however, no identified chickens in the 41BX2068 collection and only one bird bone of about chicken size.

Looking at butcher marks is another way to compare the three sites. Table A.2 shows the numbers and percentages of bones showing chop marks, hand saw cuts, and machine saw cuts in the three collections. Note that many bones had more than one type of butcher mark on them. As mentioned above, the machine saw cut bones in the Webb collection probably postdate most of the rest of that collection. Only 5.6 percent of the sawed bone in the Webb trash dump collection was machine sawed, while 39.1 percent of the sawed bone in the King/Klar privy was machine sawed. None of the bone at 41BX2068 was machine sawed.

\section{Butchering at 41BX2068}

The specifics of cutting up a carcass into appropriately sized pieces, at least by professional butchers, has changed very little in America since the mid-nineteenth century (Clonts 1983). The general sequence in processing cattle for consumption begins with slaughtering and then gutting the animal and removing the hide, head, and tail. The carcass is then split down the middle of the vertebral column, and each half is then separated into fore and hind quarters. Each quarter is then cut into smaller sections most commonly purchased by the consumer. Smaller artiodactyls such as pigs, sheep, and goats are butchered in roughly the same way.

Most of the bovid bones in this collection were from recognizable meat cuts; however, most were not cut into neat 1-inch-thick steaks and chops or 2-inch-thick roasts. For instance, most of the lumbar vertebrae of bovids in this collection were not cut into classic "T-bone" steaks. Instead, the bone was split axially down the center, usually by hand saw but occasionally by chopping, and then each half was disarticulated at each end, leaving a "T-bone" as much as 4 or 5 inches thick. While there are cut marks on many of these bones suggesting that meat had been removed from them, many had no such evidence.

Sirloin cuts (upper innominate) varied from 0.3 to 3.2 inches thick. The rump cuts (lower innominate) were also cut into a wide range of thicknesses, although there 
was more regularity in these cuts, with most less than 0.8 inches thick. There were only a few ribs with proximal or distal ends. Most were cut from medial sections.

For the most part, the femur and humerus were represented by the distal ends. Usually, the radius and ulna were cut just below the proximal epiphysis and just above the distal epiphysis, while the diaphysis was cut into lengths of approximately 5.1 to 5.5 inches long. There were no proximal tibias in this collection, but the diaphysis of the tibias was cut in a fashion similar to the radius, and there were a few distal ends. The distal femur and tibia represent hindshank cuts while the distal humerus and radius/ulna represent foreshank cuts. These are low-cost cuts. Yet the ribs, lumbar vertebrae, and dorsal innominate represent the most expensive cuts of meat available.

There were too few bones of goats and sheep to determine any sort of butchering pattern. Pig bone was largely from either feet or heads. Only one pig bone was saw cut. The rest that showed butcher marks were chopped.

In general, although the bovid bones indicate more or less standard butchering practices up to a point, there is a great deal of variation in what can be considered the end product. The standardized steaks, chops, and roasts so familiar to us today were more or less the rule at the time the trash pit was being filled, at least for professional butchers (Schulz and Gust 1983). This suggests that the butcher who processed the meat at 41BX2068 was either not professional or was butchering meat for a specific purpose that required non-standard, and for the most part larger, cuts.

\section{Bullet Holes}

Five bones in this collection had bullet holes in them (Figure A.1). All were bovid scapulas. This is an interesting finding. Generally speaking, it seems far more efficient to slaughter a cow by shooting it in the head if you must shoot it, although the professional method of slaughter at the time was an axe or hammer blow between the eyes (Clemen 1923:125-126). Why, then, were as many as five cows shot in a place that was clearly aiming at the heart? All but one of these bullet holes were from large-caliber rounds, and it is possible that an accurate shot at the heart was considered an acceptable method of slaughter. It was not, however, very professional. The potential for complications if the bullet failed to hit the heart are obvious.

Another possibility was considered during examination of the bones at the Webb trash dump (41BX897). Two bones were found that had large-caliber bullet holes in them. One, like those in this collection, was a scapula. The other bullet hole was in the transverse process of a lumbar vertebra, at an angle that made it clear the shot was made from almost directly above the cow. Since the Webb site was at the very edge of town in the early years of its habitation, it was considered possible that these cattle were hunted rather than raised (Meissner and Hunziker 1997:329-333). Until the 1930s, Texas was an open-range state, with cattle ownership determined by brands, marks, or tags. After the 1930s, many local jurisdictions required stockowners to keep their animals inside fences, although there was no state law requiring this (Dowell 2014). These days it is probably considered unneighborly 


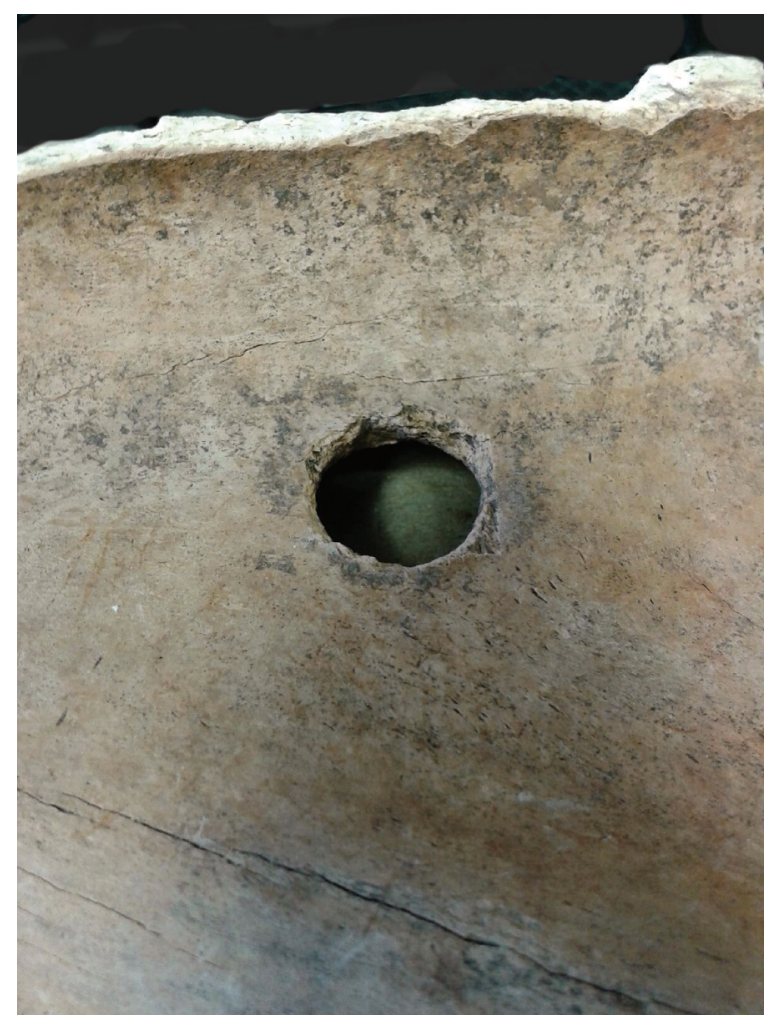

a

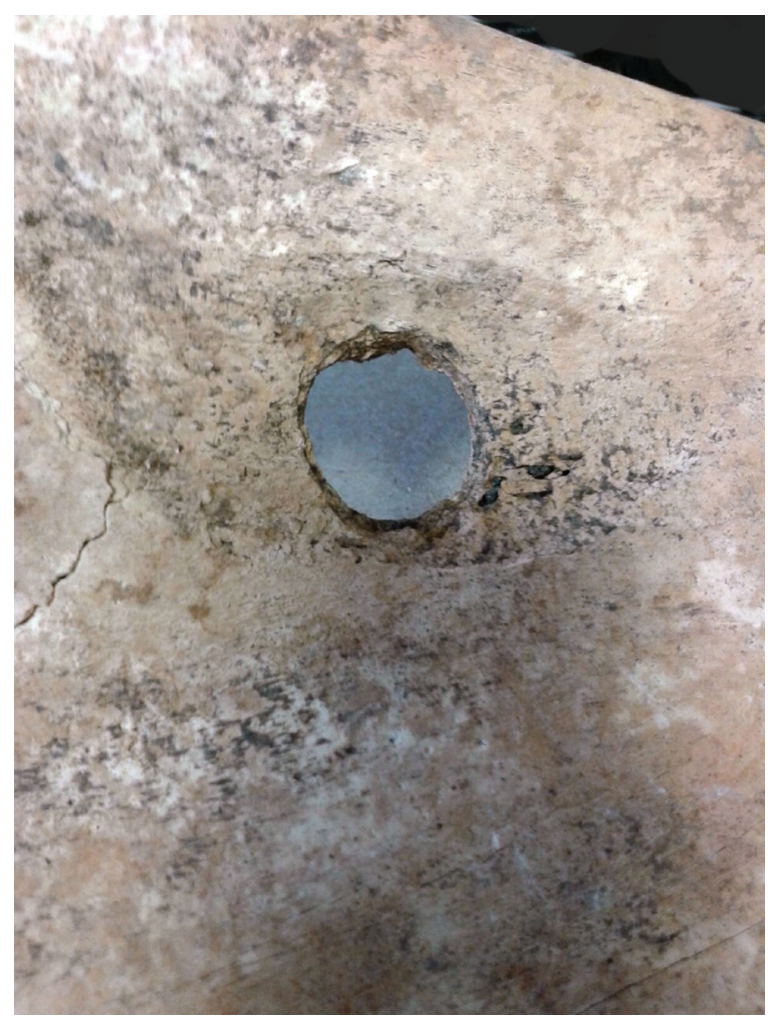

b

Figure A.1. Example of bullet hole in a bovid scapula. (a) Lateral view, showing entrance wound; (b) medial view, showing exit wound (note beveled edges).

(to say the least) to claim or kill an unmarked cow that you have not purchased, in the past cattle more than a year old with no brands, tags, or other indications of ownership were considered available to whoever caught it (McCoy 1940:82). It is possible, then, that at least some of the cattle bones found in the trash pit at 41BX2068 were from hunted animals.

In any case, the near-absence of head and lower foot bones shows that the pit was not used to dispose of waste from the early phases of cattle butchery. The presence of several head and foot bones of pigs shows that pigs might have been slaughtered at the site, although it is possible that these bones, which are considered waste on cows, were purchased in the form of pigs feet and hog jowls.

\section{CONCLUSION}

The bones found at 41BX2068 were probably deposited within a relatively short time, as there was little evidence of the bones being weathered. The identified bones are largely bovid, almost certainly all cattle (Bos taurus), with some pig and very few other identified genera. The bovids were butchered in a way that was similar to what had been common in America since at least the mid-nineteenth century, but evidence of final cuts to create the steaks, chops, and roasts with which we are familiar today, and that were commonly made by professional butchers during the 
latter part of the nineteenth century, was not often seen in this collection. While not true of every bone, many showed evidence of haphazard cutting, with many different thicknesses of cut. This suggests that the butcher was not a professional, even though the general outline of common butcher practices were followed. The near-absence of head and lower foot bones makes it clear that this was not where slaughtered animals were initially processed.

\section{REFERENCES CITED}

Balkwill, D. M., and S. L. Cumba

1992 A Guide to the Identification of Postcranial Bones of Bos taurus and Bison bison. Canadian Museum of Nature, Ottawa.

Brown, M. J., and N. De La O

1997 Beneath a Cresent Moon: A Contextual and Architectural Analysis of Privies from the Alamodome Project Area. In Archaeology at the Alamodome: Investigations of a San Antonio Neighborhood in Transition. Volume III: Artifacts and Special Studies, edited by A. A. Fox, M. Renner, and R. J. Hard, pp. 256-272. Archaeological Survey Report No. 238. Center for Archaeological Research, The University of Texas at San Antonio.

Clemen, R. A.

1923 The American Livestock and Meat Industry. Ronald Press, New York.

Clonts, J. B.

1983 Some Long Overdue Thoughts on Faunal Analysis. In Forgotten Places and Things: Archaeological Perspectives on American History, edited by A. E. Ward, pp. 349-354. Center for Anthropological Studies, Albuquerque, New Mexico.

Cohen, A., and D. Serjeantson

1996 A Manual for the Identification of Bird Bones from Archaeological Sites. Revised edition. Archetype Publications, London.

Dowell, $\mathrm{T}$.

2014 Texas Fence Law: Open Range.... or Not? Texas Agricultural Law Blog. Texas Agrilife Extension, Texas A\&M University. Electronic document, http://agrilife.org/ texasaglaw/2014/05/19/texas-fence-law-open-range-or-not-part-1, accessed May 20, 2015.

Gilbert, B. M.

1990 Mammalian Osteology. Missouri Archaeological Society, Columbia.

Gilbert, B. M., L. D. Martin, and H. G. Savage

1981 Avian Osteology. B. Miles Gilbert, Publisher, Laramie, Wyoming.

Grayson, D. K

1984 Quantitative Zooarchaeology. Academic Press, New York.

Gust, S. M.

1983 Problems and Prospects in Nineteenth Century California Zooarcheology. In Forgotten Places and Things: Archaeological Perspectives on American History, edited by A. E. Ward, pp. 341-348. Center for Anthropological Studies, Albuquerque, New Mexico.

Hillson, S.

1955 Teeth. Cambridge University Press, Cambridge, United Kingdom.

ITIS

2015 Integrated Taxonomic Information System. Electronic document, http://www.itis.gov, accessed May 17, 2015. 
Lyman, R. L.

1994 Vertebrate Taphonomy. Cambridge University Press, Cambridge, United Kingdom.

Lyman, R. L., and M. J. O'Brien

1987 Plow-Zone Zooarchaeology: Fragmentation and Identifiability. Journal of Archaeological Science 16:293-317.

McCoy, J. G.

1940 Historic Sketches of the Cattle Trade of the West and Southwest. Arthur H. Clark, Glendale, California.

Meissner, B. A., and J. M. Hunziker

1997 Analysis of the Vertebrate Faunal Remains from the Alamodome Project. In Archaeology at the Alamodome: Investigations of a San Antonio Neighborhood in Transition. Volume III:Artifacts and Special Studies, edited by A. A. Fox, M. Renner, and R. J. Hard, pp. 286-344. Archaeological Survey Report No. 238. Center for Archaeological Research, The University of Texas at San Antonio.

Olsen, S. J.

1960 Post-Cranial Skeletal Characters of Bison and Bos. Peabody Museum, Cambridge, Massachusetts.

1964 Mammal Remains from Archaeological Sites Part I; Southeastern and Southwestern United States. Peabody Museum, Cambridge, Massachusetts.

1968 Fish, Amphibian, and Reptile Remains from Archaeological Sites Part I: Southeastern and Southwestern United States. Peabody Museum, Cambridge, Massachusetts.

Reitz, E. J., and E. S. Wing

1999 Zooarchaeology. Cambridge University Press, Cambridge, United Kingdom.

Schulz, P., and S. Gust

1983 Faunal Remains and Social Status in 19th Century Sacramento. Historical Archaeology $17: 44-53$.

Tennis, C. L.

1997 Ceramic Patterns and Variations. In Archaeology at the Alamodome: Investigations of a San Antonio Neighborhood in Transition. Volume III: Artifacts and Special Studies, edited by A. A. Fox, M. Renner, and R. J. Hard, pp. 1-37. Archaeological Survey Report No. 238. Center for Archaeological Research, The University of Texas at San Antonio.

Weniger, D.

1997 The Explorers' Texas, Volume 2: The Animals They Found. Eakin Press, Austin, Texas.

Wright, G. K.

1997 Archaeology at the Alamodome: Investigations of a San Antonio Neighborhood in Transition. Volume II: Excavations and Artifact Distribution Analysis, edited by A. A. Fox, M. Renner, and R. J. Hard, Archaeological Survey Report No. 237. Center for Archaeological Research, The University of Texas at San Antonio. 
APPENDIX B: Data Compilation for Archeological Planning at HemisFair Park, San Antonio, Texas

(digital format only) 



\section{APPENDIX C: Recommendations Concerning Treatment of Archeological and Historical Resources, HemisFair Internal Streets Improvements, San Antonio, Bexar County, Texas (digital format only)}


